\title{
Adaptive Admission Control Policy for Web Caching
}

\author{
By \\ Archana Kodaypak, B.Eng.

\begin{abstract}
A thesis submitted to
the Faculty of Graduate Studies and Research

in partial fulfillment of

the requirements for the degree of

Master of Computer Science

Ottawa-Carleton Institute for Computer Science

School of Computer Science

Carleton University

Ottawa, Ontario
\end{abstract}

December 2006

C Copyright

2006, Archana Kodaypak, B.Eng. 


$\begin{array}{ll}\begin{array}{l}\text { Library and } \\ \text { Archives Canada }\end{array} & \begin{array}{l}\text { Bibliothèque et } \\ \text { Archives Canada }\end{array} \\ \begin{array}{l}\text { Published Heritage } \\ \text { Branch }\end{array} & \begin{array}{l}\text { Direction du } \\ \text { Patrimoine de l'édition }\end{array} \\ \begin{array}{l}\text { 395 Wellington Street } \\ \text { Ottawa ON K1A ON4 }\end{array} & \begin{array}{l}\text { 395, rue Wellington } \\ \text { Ottawa ON K1A ON4 } \\ \text { Canada }\end{array}\end{array}$

Your file Votre référence ISBN: 978-0-494-23355-9 Our file Notre référence ISBN: 978-0-494-23355-9

NOTICE:

The author has granted a nonexclusive license allowing Library and Archives Canada to reproduce, publish, archive, preserve, conserve, communicate to the public by telecommunication or on the Internet, loan, distribute and sell theses worldwide, for commercial or noncommercial purposes, in microform, paper, electronic and/or any other formats.

The author retains copyright ownership and moral rights in this thesis. Neither the thesis nor substantial extracts from it may be printed or otherwise reproduced without the author's permission.
AVIS:

L'auteur a accordé une licence non exclusive permettant à la Bibliothèque et Archives Canada de reproduire, publier, archiver, sauvegarder, conserver, transmettre au public par télécommunication ou par l'Internet, prêter, distribuer et vendre des thèses partout dans le monde, à des fins commerciales ou autres, sur support microforme, papier, électronique et/ou autres formats.

L'auteur conserve la propriété du droit d'auteur et des droits moraux qui protège cette thèse. $\mathrm{Ni}$ la thèse ni des extraits substantiels de celle-ci ne doivent être imprimés ou autrement reproduits sans son autorisation.
In compliance with the Canadian

Privacy Act some supporting forms may have been removed from this thesis.

While these forms may be included in the document page count, their removal does not represent any loss of content from the thesis.
Conformément à la loi canadienne sur la protection de la vie privée, quelques formulaires secondaires ont été enlevés de cette thèse.

Bien que ces formulaires aient inclus dans la pagination, il n'y aura aucun contenu manquant.

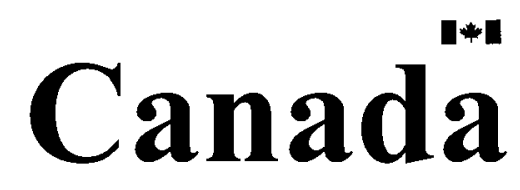




\begin{abstract}
This thesis describes the design and implementation of new admission control policies, including the Least Recently Used-Adaptive Threshold (LRU-AT) for Web caching. This policy considers the size of the requested document as an admission constraint in conjunction with the traditional LRU replacement policy. By adaptively tuning the document size, we show that the LRU-AT policy obtains a balance between the document hit ratio and the byte hit ratio. At lower cache sizes, the LRU-AT policy achieves significant improvement in the document hit ratio compared to the traditional LRU and the LRU-Threshold policies. We also show that the LRU-AT policy achieves a higher byte hit ratio when compared to the LRU-T policy. Compared to the LRU policy, we find that the LRU-AT policy has a wide variation in the byte hit ratio, which is probably due to the adaptive tuning of the size filter. At higher cache sizes, the hit ratio with the LRU-AT policy is comparable to the LRU and the LRU-T policies. As opposed to this the byte hit ratio with the LRU-AT policy is significantly higher than that yielded by the LRU-T policy and comparable to the LRU policy.
\end{abstract}




\section{Acknowledgments}

I would like to thank my Supervisor, Dr. John B. Oommen, for his research guidance and encouragement for the later part of the completion of this Thesis primarily after the tragic departure of Prof. Sivarama Dandamudi.

I would also like to sincerely thank my Co-Supervisor, the late Dr. Sivarama P. Dandamudi, for his guidance on Web Caching related research, and his personal encouragement for the major part of this research. I miss you, Sir. I am also grateful to him for providing me the opportunity to personally present the results of my Thesis at two International Conferences, the World Multi-Conference on Systemics, Cybernetics and Informatics (WMSCI-2005) in Orlando, and the International Conference on Intelligent and Adaptive Systems and Software Engineering (IASSE-2005) in Toronto respectively.

Trace data used in our simulations were collected by the National Laboratory for Applied Network Research. Thanks to Dr. Duane Wessels for making the July 2004 traces obtained from various proxies, available.

I am indebted to Carleton University and the Natural Sciences and Engineering Research Council (NSERC) of Canada for providing me with partial financial support for this research. 


\section{Table Of Contents}

1 INTRODUCTION 1

1.1 Motivation 1

1.2 WORKLOAD CHARACTERISTICS 3

1.3 THESIS OBJECTIVE

1.4 CONTRIBUTION OF THE THESIS

1.5 OUTLINE OF THE THESIS

2 BACKGROUND INFORMATION AND PREVIOUS WORK 6

2.1 Web CACHING - GeNeral Model 6

$\begin{array}{lll}\text { 2.1.1 Proxy Caching } & 7\end{array}$

2.1.2 Prefetching 12

$\begin{array}{lll}2.2 & 15\end{array}$

$\begin{array}{lll}2.3 & \text { REPLACEMENT POLICIES } & 23\end{array}$

2.3.1 Traditional replacement policies 23

2.3.2 Key-based replacement policies $\quad 25$

2.3.3 Cost-based replacement policies 26

2.4 PREVIOUS WORK ON WEB WORKLOADS 31

2.4.1 Web Workload Characteristics

2.4.2 Adaptive Feedback admission control policy 32

3 ONE-TIMER AND NON-ONE TIMER ANALYSIS 34

$\begin{array}{lll}3.1 & \text { INTRODUCTION } & 34\end{array}$

3.2 Data Collection, Reduction ANd ANALysis 35

3.2.1 Data Collection 35

3.2.2 Data Reduction $\quad 37$

$\begin{array}{lll}\text { 3.2.3 } & \text { Trace Statistics } & 38\end{array}$ 
3.2.4 Data Analysis $\quad 39$

$3.3 \quad$ TRANSFER SIZE CHARACTERISTICS 41

3.3.1 Mathematical Models 44

3.3.2 One-Timer and Non-one Timer Analysis 46

$\begin{array}{lll}\text { 3.3.2.1 One-Timer Documents } & 47\end{array}$

3.3.2.2 Non-One Timer Documents 52

$\begin{array}{lll}\text { 3.3.2.3 Total Documents } & 56\end{array}$

3.3.3 Goodness of Fit $\quad 61$

3.3.4 Heavy Tailed Property 63

$\begin{array}{lll}3.4 & \text { POPULARITY CHARACTERIZATION } & 68\end{array}$

$\begin{array}{lll}3.5 & \text { SUMMARY } & 74\end{array}$

4 ADAPTIVE ADMISSION CONTROL POLICY 76

$\begin{array}{lll}4.1 & \text { INTRODUCTION } & 76\end{array}$

$\begin{array}{lll}4.2 & \text { REPLACEMENT POLICIES } & 77\end{array}$

$\begin{array}{lll}\text { 4.2.1 Effect of Cache Size } & 78\end{array}$

$\begin{array}{lll}\text { 4.2.2 Performance Metrics } & 79\end{array}$

4.3 ADAPTIVE FEEDBACK POLICY 80

4.4 Simulation AND Results

$\begin{array}{lll}\text { 4.4.1 Cache Sizes } & 84\end{array}$

$\begin{array}{lll}\text { 4.4.2 Cache Management Policies } & 87\end{array}$

$\begin{array}{lll}\text { 4.4.3 Results } & 88\end{array}$

$\begin{array}{lll}\text { 4.4.3.1 } & \text { Byte hit ratio } & 95\end{array}$

$\begin{array}{lll}4.5 & \text { SUMMARY } & 103\end{array}$

5 CACHING USING AUTOMATA-BASED PARTITIONING 105

$\begin{array}{lll}5.1 & \text { INTRODUCTION } & 105\end{array}$

5.2 ADAPTIVE LINEAR LISTS 106

$\begin{array}{lll}5.3 & \text { LIST-ON-LIST ORGANIZATION } & 108\end{array}$ 
5.5 CONCLUSIONS

6 CONCLUSIONS AND FUTURE WORK

$\begin{array}{llr}6.1 & \text { CONTRIBUTIONS OF THE THESIS } & 114\end{array}$

$\begin{array}{ll}6.2 & 117\end{array}$

7 REFERENCES 119 


\section{List Of Figures}

Figure 3.1: CDF of total unique documents for NLANR traces

Figure 3.2: PDF of total unique documents for NLANR traces 44

Figure 3.3: CDF of One-Timer documents for UC1 trace $\quad 48$

Figure 3.4: CDF of One-Timer documents for UC2 trace 49

Figure 3.5: CDF of One-Timer documents for BO1 trace

Figure 3.6: CDF of One-Timer documents for $\mathrm{BO} 2$ trace $\quad 50$

Figure 3.7: CDF of One-Timer documents for PA trace $\quad 50$

Figure 3.8: CDF of One-Timer documents for PB trace

Figure 3.9: CDF of One-Timer documents for NY trace

Figure 3.10: CDF of Non-one Timer documents for UC1 trace 53

Figure 3.11: CDF of Non-one Timer documents for UC2 trace

Figure 3.12: CDF of Non-one Timer documents for BO1 trace

Figure 3.13: CDF of Non-one Timer documents for BO2 trace $\quad 54$

Figure 3.14: CDF of Non-one Timer documents for PA trace 55

Figure 3.15: CDF of Non-one Timer documents for PB trace 55

Figure 3.16: CDF of Non-one Timer documents for NY trace 56

Figure 3.17: CDF of Total documents for UC1 trace 58

Figure 3.18: CDF of Total documents for UC2 trace 58

Figure 3.19: CDF of Total documents for BO1 trace

Figure 3.20: CDF of Total documents for $\mathrm{BO} 2$ trace

Figure 3.21: CDF of Total documents for PA trace $\quad 60$

Figure 3.22: CDF of Total documents for PB trace $\quad 60$ 
Figure 3.23: CDF of Total documents for NY trace

Figure 3.24: LLCD of documents size for UC1 trace

Figure 3.25: LLCD of documents size for UC2 trace

Figure 3.26: LLCD of documents size for BO1 trace

Figure 3.27: LLCD of documents size for $\mathrm{BO} 2$ trace

Figure 3.28: LLCD of documents size for PA trace

66

Figure 3.29: LLCD of documents size for PB trace $\quad 66$

Figure 3.30: LLCD of documents size for NY trace $\quad 67$

$\begin{array}{ll}\text { Figure 3.31: Popularity vs rank for UC1 trace } & 70\end{array}$

Figure 3.32: Popularity vs rank for UC2 trace $\quad 70$

Figure 3.33: Popularity vs rank for BO1 trace $\quad 71$

Figure 3.34: Popularity vs rank for BO2 trace $\quad 71$

Figure 3.35: Popularity vs rank for PA trace $\quad 72$

Figure 3.36: Popularity vs rank for PB trace $\quad 72$

Figure 3.37: Popularity vs rank for NY trace

Figure 4.1: Adaptive Feedback Admission Control Policy Algorithm used by the $\begin{array}{ll}\text { Simulation Model } & 83\end{array}$

Figure 4.2: Hit ratio vs Cache size for UC1 trace 90

Figure 4.3: Hit ratio vs Cache size for UC2 trace $\quad 91$

Figure 4.4: Hit ratio vs Cache size for BO1 trace $\quad 91$

Figure 4.5: Hit ratio vs Cache size for $\mathrm{BO} 2$ trace $\quad 92$

Figure 4.6: Hit ratio vs Cache size for PA trace 92

Figure 4.7: Hit ratio vs Cache size for PB trace 93

viii 
Figure 4.8: Hit ratio vs Cache size for NY trace

Figure 4.9: Byte hit ratio vs Cache size for UC1 trace

Figure 4.10: Byte hit ratio vs Cache size for UC2 trace

Figure 4.11: Byte hit ratio vs Cache size for BO1 trace

Figure 4.12: Byte hit ratio vs Cache size for $\mathrm{BO} 2$ trace

100

Figure 4.13: Byte hit ratio vs Cache size for PA trace

Figure 4.14: Byte hit ratio vs Cache size for PB trace

Figure 4.15: Byte hit ratio vs Cache size for NY trace 


\section{List Of Tables}

Table 3.1: Statistics of the raw data for NLANR traces 36

Table 3.2: Statistical properties of clean log data for NLANR traces 38

Table 3.3: Statistics of One-Timer documents in NLANR traces 40

Table 3.4: Weibull fitted parameters for One-Timer CDF 48

Table 3.5: Lognormal fitted parameters for One-Timer CDF 48

Table 3.6: Weibull fitted parameters for Non-one Timer CDF 52

Table 3.7: Lognormal fitted parameters for Non-one Timer CDF 52

Table 3.8: Weibull fitted parameters for Total documents CDF 57

Table 3.9: Lognormal fitted parameters for Total documents CDF 57

Table 3.10: Goodness of fit $d_{n}$ values for one-timer, non-one timer and total documents

Table 3.11: Estimate of $\alpha$ for size of One-Timer, Non-one Timer and Total documents 68

$\begin{array}{ll}\text { Table 3.12: Popularity characteristics for all NLANR traces } & 74\end{array}$

Table 4.1: Statistics for seven NLANR traces $\quad 85$ 


\section{Introduction}

The emergence of a World Wide Web Revolution has resulted in the globalization of information access and in providing a wide range of Internet-based static and on-demand services, including shopping, banking and e-commerce, etc. Consequently, the volume of transported WWW traffic has been increasing, and continues to do so at a rapid pace. Such a growth has resulted in the telecommunications core transport and edge access networks become prone to congestion and this has, in turn increased the traffic load on Web servers, thereby resulting in an increase in the access response times of a wide-variety of WWW documents. As multimedia content delivery networks, on-demand applications and services continue to grow in the Wired and Wireless Internet world, the latency to deliver such mission critical and cost-cum-quality sensitive services to businesses and consumers becomes even more significant.

\subsection{Motivation}

Caching has been widely used in memory architectures to improve computer performance. Web caching is similar to the concept of memory system caching - a Web cache stores Web resources in anticipation of future requests. Due to a variety of factors including the non-uniformity of Web object sizes, retrieval costs and cacheability, there are significant differences between memory system caching and Web caching. Web caching is a significant part of the Web's infrastructure as it reduces network traffic and 
Web server loads. WWW caching addresses the user-perceived latency problem by bringing documents closer to the clients.

Caching is deployed at various locations (at the client, the server and the proxy server) [3]. The placement of the cache has been less studied compared to caching policies themselves. The proxy server is a computer that is placed near a gateway to the Internet. Client requests arrive at the proxy regardless of the Web servers that host the required document. The proxy either serves these requests using previously cached responses, or requests the document from the origin server on behalf of the client. It stores the responses in its cache for future use. A proxy cache has limited storage. When the cache is full, a caching policy decides which documents have to be purged to allow new documents. This caching policy is called a replacement policy. Several replacement policies have been proposed in the literature [4]. These policies attempt to optimize various Web performance metrics including the document-hit ratio, the byte hit ratio, the response time etc.

Earlier studies have dealt with replacement policies that considered static admission constraints (such as the Least Recently Used-Threshold) in improving the cache performance. Indeed, dynamic admission constraints have never been dealt with in detail while evaluating cache performance. As traffic patterns continue to grow and evolve, such constraints may help in designing adaptive replacement policies that are effective in improving the performance of the cache. The objective of this Thesis is to understand the nature of such Web workloads and system demands created by WWW users. By using the document size as an adaptive admission constraint in conjunction with the LRU 
replacement policy, the cache performance is measured and compared against the static policies.

\subsection{Workload Characteristics}

Understanding the dynamic nature of workloads, and the system demands created by users of the WWW, is critical to improve the performance of the Web. Web proxy workload characteristics and proxy caching have been well studied in the literature. Some common characteristics that have been identified in proxy workloads are:

- The document popularity which often can be approximated to follow Zipf's law [5]

- The distribution of the file size for web documents which is heavy tailed [6]

- Many (50\%-70\%) documents are referenced only once [7]

- Temporal locality exists in web proxy reference streams [8]

These characteristics affect the design of the proxy cache system.

Documents that are requested only once are called one-timer documents, whereas those that are requested multiple times are called non-one timer or popular documents. The percentage of one-timer and non-one timer documents requested varies from trace to trace in a hierarchical cache structure. Regardless of the duration of the access logs studied in the past, it has been found that one-timer documents are predominant [9]. Earlier studies have shown that most Web document transfers are small with the mean document size being between 7 and 15 Kbytes, and the median transfer size being around 
2 to 3 Kbytes. However, no clear distinction has been made between one-timer and nonone timer documents by studying their file transfer size distributions.

\subsection{Thesis Objective}

This Thesis presents a detailed study of the Web workload characteristics in terms of onetimers, and non-one timers, and their individual file size distributions using empirical and mathematical models. These models further help us in understanding the impact of the file size, that, in turn, is used as an admission constraint parameter in conjunction with a cache replacement policy to assess the overall performance of the cache. We propose a new admission control replacement policy, called the "Least Recently Used-Adaptive Threshold (LRU-AT)", so as to study the cache performance. This policy takes into account the size of the requested documents and optimizes the document replacement strategy, thereby providing a balance between the documents hit ratio and the byte hit ratio.

\subsection{Contribution of the Thesis}

The motivation behind developing the LRU-AT policy stems from the interest in having a learning system or technique that is able to use the document size as an admission constraint. This can be adaptively tuned, based on a trace partitioning, to hopefully achieve an optimal cache performance. As there are very few techniques based on adaptive admission constraints, the primary goal is to develop such a scheme, and to experimentally compare it to other replacement policies. 


\subsection{Outline of the Thesis}

The rest of the Thesis is organized as follows. Chapter 2 presents a background study on Web caching, workload characteristics and different replacement policies, and gives a brief overview of how our work is different from the previous studies. Results of transfer file size distribution of all the traces in terms of one-timers and non-one timers are presented in Chapter 3. The simulation and results of the new replacement policy, the adaptive feedback LRU based admission control policy is discussed in Chapter 4. In Chapter 5 , we propose a strategy by which these caching policies can be further enhanced by incorporating it in conjunction with a learning automaton ${ }^{1}$. A summary of the experimental results and conclusions are presented in Chapter 6, followed by some pointers to additional research in Web caching.

\footnotetext{
${ }^{1}$ Unfortunately, due to time constraints and the tragic demise of Professor Sivarama Dandamudi, much of this work will be left for a future project.
} 


\section{Background Information and Previous Work}

The main thrust of this Thesis is to study the design of replacement algorithms for improving the performance of Web caching. Section 2.1 presents a general model of Web caching which is widely documented in the literature, on which our subsequent discussions are based. Secondly, we describe Web workload characteristics in Section 2.2. Section 2.3 provides an overview of the various replacement policies that are currently used in Web caching. In Section 2.4 , we review the previous work related to this Thesis.

\subsection{Web Caching - General Model}

The explosive growth in the popularity of the World Wide Web has contributed significantly to the network traffic on the Internet. This has led many researchers to be motivated to improve the performance and scalability of the Web. Some of the commonly used methods to improve the Web performance are summarized below.

Web Server Improvement: This strategy primarily deals with improving the Web server response time associated with the client's requests. The performance improvement of Web servers is an active area of research [10]. One solution for reducing server response times is to resort to the use of parallel Web server hardware. For example, IBM had used the SP2 parallel system in the design of Web Servers for the 1998 Olympic Winter games [10]. A cluster of workstations that can share cache information and cache data between 
the nodes was used by the Alexandria Digital Library (ADL) for implementing a Web server [11]. Other researchers have tried to reduce network bottlenecks through load balancing on multi-node Web servers. Processing capacity rather than network bandwidth is the performance bottleneck for sites that use dynamic pages [10].

Proper Design of Web Pages: Web performance has also been improved through the establishment of guidelines for the proper design of Web pages that lead to a more efficient system. The number, size and type of documents (high quality audio/video) embedded in the front pages of popular Web sites affect the Web performance significantly and could lead to larger download times.

Employing Caching: Caching is a popular and effective technique that has been widely used to improve Web performance. Caching has been proven to be effective, inasmuch as a small fraction of the total unique documents accessed on the Web often account for a large fraction of document referenced. By caching the most popular documents closer to the clients requesting them, network traffic can be greatly reduced.

\subsubsection{Proxy Caching}

Caching is deployed in several places including at the client, at the server or at the proxy server [3]. In this work we focus on proxy caching, in which one or more computers act as a cache for a set of WWW clients. Proxy caching improves the Web performance by reducing the user latency while requesting Web documents. This is due to the fact that the proxy cache is located closer to the client than the content provider. This proximity to the 
client lowers the network traffic as well. Also, most of the cache hits are directed to the proxy, which lowers the service demand on the Web servers.

Proxy caching can be implemented using a single-level or a multi-level hierarchical proxy caching philosophy. A multi-level cache can be assumed to have four different levels: lower, institutional, regional and national levels [12]. At the lower level of the hierarchy there are the client/browser caches. When the client cache does not satisfy a request, the request is redirected to the institutional cache. If the document is not found at the institutional level, the request is then forwarded to the regional level cache, which, in turn, forwards unsatisfied requests to the national level cache. If the document is not found at any cache level, the national level cache contacts the origin server. When the document is found, either at a cache or at the origin server, it travels down the hierarchy, leaving a copy at each of the intermediate caches along its trajectory. Further requests for the same document travel up the caching hierarchy until the document is hit at some cache level.

\section{Hierarchical Web Caching}

Hierarchical Web caching was first proposed in the Harvest project [13]. While the hierarchical architecture is more bandwidth efficient, it has some problems indicated below:

1. In order to setup a hierarchy, cache servers need to be placed at key access points in the network. This requires coordination between the participating cache servers. 
2. Delays could be incurred at each level of the hierarchy.

3. High level caches may become bottlenecks and have long queuing delays.

4. Multiple copies of the same document are stored at different cache levels.

Roadknight et al. [14] reported that the effect of lower-level proxy caches is to reduce the temporal locality of requests for higher-level caches. This implies that the hit ratio for the proxies that are located at higher levels of the cache hierarchy is less than that associated with the proxies at the lower levels of the hierarchy. The National Laboratory for Applied Network Research (NLANR) [15] manages IRCACHE that operates in the U.S. IRCACHE is a global cache that consists of parent and sibling caches. Connecting to IRCACHE can improve the performance of caching [16]. For communication and cooperation of proxies in a hierarchy of caches, several protocols have been proposed. Some of these approaches are explained here.

The common way for organizing a hierarchy of proxies is referred to as Static proxy caching hierarchy. The Internet Cache Protocol (ICP) [17] is a common protocol that provides communication among the proxies in a hierarchy of caches. The ICP is a lightweight message format, and is implemented on top of the UDP to guarantee a quick query/reply message exchange. It is used to provide hints about the existence of URLs in the related caches in the hierarchy. Harvest caches and Squid [18] use ICP messages to contact other proxy caches in a hierarchy. For example, if the Squid cache cannot find a requested document, it sends an ICP query to its siblings, and the siblings respond with ICP replies that indicate a HIT or a MISS. Based on this result, Squid retrieves the 
document from the siblings, parent or original site [19]. More details about the ICP are presented in [20], [21].

Besides the static hierarchy of proxy caching, another approach for using proxy caches in a hierarchy is the dynamic approach. Chiang et al. [22] proposed a Caching Neighborhood Protocol (CNP) that describes an infrastructure by which proxy servers are able to build dynamic Web caching hierarchies. In the CNP, if the requested file is not available in a proxy cache close to the client, this proxy uses its request-forwarding table to select a server to which it forwards this request. The request-forwarding table contains information about all the proxies that are representative of the original server for that requested document. The request-forwarding table also contains the estimate of the request latency response for each of the proxies. More details about the CNP are presented in [22].

\section{Distributed Web Caching}

In distributed Web caching systems [23], there are no other intermediate cache levels than the institutional caches, which serve each other's Misses. In order to decide from which institutional cache a Missed document is to be retrieved, all institutional caches keep meta-data information about the content of every other institutional cache. To make the distribution of the meta-data information more efficient and scalable, a hierarchical distribution mechanism can be employed. However, the hierarchy is only used to distribute directory information about the location of the documents, and not for retaining copies of the actual documents themselves. With distributed caching, most of the traffic 
flows through low network levels, which are less congested, and thus additional disk space is required at intermediate levels. In addition, distributed caching yields a superior load sharing, and is thus more fault tolerant. Nevertheless, it has been reported that a large-scale deployment of distributed caching may encounter several problems such as high connection times, higher bandwidth usage, administrative issues, etc. [12].

Several approaches exist for distributed caching. Besides the ICP designed by the Harvest group [17], another approach to distributed caching is the Cache Array Routing Protocol (CARP) [24], which divides the URL-space among an array of loosely coupled caches and lets each cache store only the documents whose URLs are hashed to it. Provey and Harrison also proposed a distributed Internet cache [23]. In their scheme, upper level caches are replaced by directory servers, which contain location hints about the documents kept at every cache. A hierarchical meta-data-hierarchy is used to make the distribution of these location hints more efficient and scalable. Tewari et al. proposed a similar approach to implement a fully distributed Internet caching system where location hints are replicated locally at the institutional caches [25]. In the central directory approach (CRISP) [26], a central mapping service ties together a certain number of caches. Similarly, in the Cachemesh system [27], cache servers establish a cache routing table among themselves, and each cache server becomes the designed server for a number of Web sites. User requests are then forwarded to the proper cache server according to the cache routing table. In Summary Cache [28], cache digest [29], and the Relias project [30], caches inter-exchange messages indicating their content and keep local directories to facilitate finding documents in other caches. 


\section{Hybrid Web Caching}

In hybrid architecture, caches may cooperate with other caches at the same level or at a higher level using distributed caching. The ICP [17] is a typical example for this phenomenon. The document is fetched from a parent/neighbor cache that has the lowest Round Trip Time (RTT). Rabinovich et al. [31] proposed to limit the cooperation between neighbor caches to avoid obtaining documents from distant or slower caches, which could rather have been retrieved directly from the origin server at a lower cost.

\subsubsection{Prefetching}

Although the performance of the Web can be improved by caching documents at proxies, the benefit from this technique is limited. Previous research has shown that the maximum cache hit rate that can be achieved by any caching algorithm is usually no more than $40 \%$ to $50 \%$. In other words, regardless of the caching scheme, unfortunately, one out of two documents cannot be found in the cache [32]. One way to further increase the cache hit rate is to anticipate future document requests, and to preload or prefetch these documents in a local cache. In the context of Web, prefetching can be applied in three ways:

1. Between browser clients and Web servers

2. Between proxies and Web servers

3. Between browser clients and proxies 


\section{Prefetching between browser clients and Web servers}

Early studies focused on the prefetching schemes between browser clients and Web servers. Padmanabhan and Mogul [33] analyzed the latency reduction and network traffic of prefetching using Web server traces and trace-driven simulations. Their study demonstrated that prefetching from Web servers to individual clients can reduce the client latency by $45 \%$ at the expense of doubling the network traffic. Bestavros and Cunha [34] presented a model for speculative dissemination of WWW documents. Their work demonstrated that reference patterns observed at a Web server can be used as an effective source of information to drive prefetching, and yielded results similar to those reported in [35]. Cunha and Jaccoud used [36] collections of Web client traces and studied how effectively a User's future Web accesses can be predicted from his or her past Web accesses. Crovella and Barford [37] analyzed the network effects of prefetching and showed that prefetching can reduce access latency at the cost of increasing network traffic and burstiness. However, these early studies did not consider or model caching proxies, and hence failed to answer the question about the performance of prefetching completely.

\section{Prefetching between proxies and Web servers}

Kroeger et al. [38] investigated the performance limits of prefetching between Web servers and proxies, and showed that combining caching and prefetching at the proxies can reduce the client latency by $60 \%$ for high bandwidth clients. Markatos and Chronaki [39] proposed that Web servers regularly push their most popular documents to Web 
proxies, which then, in turn push those documents to the clients. They evaluated the performance of the strategy using several Web server traces and determined that this technique, with cooperation from the respective Web servers, can anticipate more than $40 \%$ of a client's request. Cohen et al. [40] also investigated similar techniques. Wcol [41] is proxy software that prefetches documents, links, and embedded images. The proxy however does not push documents to the client. Gwertzman and Seltzer [42] discussed a technique called Geographical Push-Caching where a web server selectively sends its documents to the caches that are closest to its clients. The focus of the study was on deriving reasonably accurate network topology information and using the information to select caches.

\section{Prefetching between browser clients and proxies}

Prefetching can also be done between browser clients and proxies. Loon and Bharghavan [43] proposed a design and an implementation of a proxy system that performs the prefetching and the image filtering for mobile clients. Fan et al. [44] proposed an approach to reduce latency by prefetching between caching proxies and browsers. The approach relies on the proxy to predict which cached documents a user might reference next and takes advantage of idle time between user requests to either push or pull the documents to the user. Simulation results show that prefetching combined with larger browser cache and delta-compression can reduce client latency up to $23.4 \%$. 


\subsection{Web Workload Characteristics}

Understanding Web traffic characteristics is a key to the successful design of techniques that conserve network bandwidth, reduce latency, and improve response time of Web accesses. Researchers in the past have identified Web workload characteristics by analyzing Web server access logs from various sources including academic (i.e., university) environments, scientific research organizations and commercial Internet providers. The duration of these access logs studied varied considerably, ranging from a day of activity to one year of activity or more. Some studies have also considered access logs collected from different levels of cache hierarchies. Also, based on the characteristics and distributions of Web requests, researchers have developed tools for simulating Web requests to analyze the performance of the Web.

For example, Arlitt and Williamson [46] identified ten workload invariants by analyzing six server logs. Mahanti and Williamson [50] [51] also studied workload characteristics by analyzing three access logs from different levels of cache hierarchy. Busari and Williamson [48] designed a Web proxy workload generator called ProWGen, in order to evaluate proxy cache performance in multi-level caching hierarchies.

In the following subsections, a more detailed analysis of the Web proxy workload characteristics is presented.

\section{Document Types}

Document requests in access server logs are typically classified into the following generic categories: 
- HTML (e.g., .html, .shtml, .htm)

- Image (e.g., .gif, .jpeg, .gif89, .xbm)

- $\quad$ Audio (e.g., .au, .ram, .wav)

- Video (e.g. .mpeg, .avi, .mov)

- Text (e.g., .tex, .readme, .c, .java)

- Compressed (e.g., .zip, .gz)

- Application (e.g., .ps, .pdf, .dvi)

- Dynamic (e.g., .cgi, .perl)

The analysis of Mahanti and Williamson [50] was based on three access server logs from different cache hierarchies, and this has shown that HTML and Image files accounted for $95 \%$ of the total document requests. Similar results were reported for client traces by Cunha et al. [49] and for server traces by Arlitt and Williamson [47]. In [47], it was found that Image files accounted for $65-80 \%$ followed by HTML files (17-28\%). Similar observations were made by Abdull et al. [7] and Abhari [89] in their characterization of Web proxy traffic.

\section{Transfer Size Distribution}

Documents returned to the requesting clients, either directly by the proxy, or after obtaining the document from a higher-level proxy, or from the originating server, have certain transfer size characteristics. The size of these requested documents could vary from a few bytes to Megabytes or Gigabytes depending on the trace. 
Arlitt and Williamson [47] demonstrated in their trace analysis that all of the transfer sizes for each proxy server are in the range from 100 to 100,000 bytes, with very few small transfers below 100 bytes and very few large transfers above 100,000 bytes. They also showed that their file size distribution was heavy-tailed, which was similar to the file size distribution reported for Web clients [45] and for Web servers [47]. Similar observations were made by Mahanti and Williamson [50] by analyzing other data sets.

A "heavy tail" in a Web document size distribution indicates the presence of very large "elephants" (i.e. outliers) in the tail of the distribution (although few in number) are large enough to contribute significantly to the overall traffic volume. The simplest example of a heavy-tailed distribution is the doubly-exponential Pareto distribution. The cumulative distribution function for the Pareto distribution is:

$$
F(x)=1-\left(\frac{k}{x}\right)^{\alpha}, k>0, x \geq k
$$

The $\alpha$ parameter is known as the tail-index [6], and $k$ defines the beginning of the tail of the distribution. As $\alpha$ decreases, the tail of the distribution becomes heavier. In other words, an arbitrarily large portion of the probability mass may be present in the tail of the distribution as $\alpha$ decreases. To estimate the tail index $\alpha$, a $\log$-Log Complementary Distribution (LLCD) is plotted for the transfer sizes in the data sets. An LLCD plot graphs $\log \bar{F}(x)=\log (1-F(x))$ vs $\log (x)$, for large $x$ [6]. Heavy tailed distributions have the following property:

$$
\frac{d \log \bar{F}(x)}{d \log x}=-\alpha, x>k
$$


An estimate of the tail index $\alpha$ is obtained by determining the slope of the LLCD plot for values of $x$ greater than $k$, using a least-squares linear regression. A "goodness of fit" test is usually performed to assess the linear regression. In [51], Mahanti and Williamson concluded that proxy transfer sizes are heavy tailed just like server transfer sizes. They also observed that caching smaller documents at lower-level proxies resulted in heavier tails at higher-level caches.

\section{File Popularity}

Popularity of Web documents has been noticed by many researchers [6], [47] and [49] to be highly uneven, and Zipf's Law [52] has been applied to model this behavior. Zipf's Law states that if items are ranked $(r)$ according to their popularity $(P)$, then the popularity of an item is inversely proportional to its rank.

The Zipf distribution is a parameter-less hyperbolic distribution of the form:

$$
P \sim\left(\frac{1}{r}\right)^{-\beta}
$$

where the exponent $\beta$ is often close to unity [14].

In the special case, where the exponent $\beta=1$, the $\mathbf{n}^{\text {th }}$ most popular item is exactly twice as popular as the 2 nth most popular item, and so on [53]. Zipf distribution implies that a few documents are very highly referenced, a moderate number of documents are moderately referenced, while a large number of documents are referenced only a few times. To see whether or not the documents follow the Zipf distribution, the documents 
are first sorted in descending order according to their frequency of reference. The documents are then ranked, with the most referenced document being assigned a rank of unity, followed by the next most referenced document with a rank of two, and so on. A $\log -\log$ plot of the document reference count versus rank is then obtained for the data set and using goodness of fit test, the exponent of the Zipf distribution is determined. For proxy workloads, Arlitt et al. [47] reported that the value $\beta$ ranges between 0.5 and 1 .

\section{Temporal Locality}

Temporal locality refers to the property that an object referenced in the recent past will likely be referenced again in the near future. This is an important characteristic of the system because cache effectiveness can be significantly increased when temporal locality is present in the file references. Temporal locality is measured using a Least Recently Used (LRU) stack model [54]. The LRU Stack Model (LRUSM) is a stack-based ordering of referenced objects according to their recency of reference (i.e., the most recently referenced item is placed on top (position 1), and the least recently referenced item on the bottom). For each reference in the request stream, the stack is searched until the requested object is found, or the bottom of the stack is reached. If the document is a Hit, the object is removed from its present position in the stack (say, d) and moved to the top of the stack, pushing the other d-1 items that used to be above it down one position, as needed. For a document Miss, it is simply added to the top of the stack, pushing all other stack items down one level. The most important part of the LRUSM is keeping track of the stack depth $d$ at which each Hit occurs. The presence of temporal locality manifests itself in a large number of Hits at or near the top of the stack. In [49], it was 
noted that the temporal locality at proxy servers, in general, is not very high compared to temporal locality results for Web servers [47] due to the caching effects at various levels in the cache hierarchy or due to the document referencing behavior generated by the clients.

\section{One-Timer Referencing}

A surprising observation made in previous analysis of Web server workloads was that (regardless of the duration of the trace) typically 15-30 percent of the documents accessed in the $\log$ were accessed only a single time. This so-called one-timer referencing behavior is of concern for Web caching, since there is no point caching something that will be accessed only once.

The precise cause of this one-timer referencing behavior is not fully understood. Several explanations have been proposed. First, it might indicate the vastness of the Web and the low signal-to-noise ratio, where the latter is quantified by the ratio of the number of pertinent documents to the number of non-pertinent ones which will have to be cached [51]. Second, it might reflect human nature in browsing habits (e.g. once a site has been visited, there is no need to visit it again). Third, it might reflect the behavior of the content providers, who might use data-based URL names, or redesign or modify web pages on a regular basis to keep them current. Fourth, it may reflect the presence of search engines or Web robots that traverse many pages to construct an index. Fifth, the presence of a high percentage of one-timers might indicate that caching hierarchies are actually working well. Finally, it may be the consequence of document prefetching 
algorithms in some proxies and/or client browsers. All of these hypotheses seem plausible. If any (or all) of them are true, they indicate a challenging workload environment for Web caching algorithms. Mahanti and Willaimson [51] had shown that one-timers account for a substantial portion of total requests, and total bytes transferred. Also, approximately 70 percent of the documents referenced are one-timers. The predominance of one-timer referencing for Web documents highlights the need for novel Web proxy caching policies that can effectively discriminate against one-timers. For example, frequency-based algorithms, such as Least Frequently Used (LFU), tend to perform better than recency- based algorithms, such as Least Recently Used (LRU) [53]. Similar observations have been made for Web server caching algorithms [46], [47] and $[56]$.

\section{Self-Similarity}

Self-Similarity can be defined informally as follows: Looking at the data with different time resolutions does not change the underlying access distribution. In other words, the data has properties similar to fractals, where you can "zoom in" into the fractal and see the same structure. Crovella and Bestavros [57], in their study based on file size distributions, have shown that the WWW traffic exhibits self-similarity. Abdulla et al [7] performed tests for self similarity on the collected data and proved that the workloads are self similar. Arlitt and Williamson conducted several tests to check long range dependence and self-similarity in the Web server workload. 


\section{Geographic Location}

The Geographic location of the sites that requested documents is another workload invariant identified by Arlitt and Williamson in their study [45]. The IP addresses of the sites that requested documents were identified by analyzing Web server logs. The network component of the IP address was used to determine if a requesting document was from a local or remote site (relative to Web server). The network identifier in each IP address was further used to classify requesting hosts into domains that had the same network address. It was shown that remote sites accounted for more than $70 \%$ of requested accesses to the server and transferred data. Also, their study showed that Web servers are accessed by thousands of domains, with approximately $10 \%$ of the domains accounting for more than $75 \%$ of accesses.

One of the biggest obstacles still facing Web characterization is that of instrumentation. Access to most recent representative traces from the client, proxy and server access points remains difficult. The majority of characterizations to date focus heavily on US education settings, resulting in a scarcity of client characterization from commercial Internet Service providers, corporate environments, and the international user community. As these sectors represent significant portions of today's WWW growing traffic, access to such information would help us in better understanding different traffic usage patterns. Another shortcoming of the existing research in this field is that many of the studies have been outdated. Thus, analyzing current traffic patterns from various traces (educational and commercial) is absolutely important to understand, validate and further refine the Web performance algorithms studied in the past. With better 
instrumentation available at the server, network, proxy, and client access points, it would allow us to better characterize Web traffic and improve its performance with reduced latency and better end-user satisfaction.

\subsection{Replacement Policies}

A document placement/replacement algorithm that can yield high hit rate often determines the effectiveness of the proxy cache. A number of cache replacement policies have been proposed in recent studies, which attempt to minimize various cost metrics, such as the hit rate, byte hit rate, average latency, and total cost. They are broadly classified into the following three categories, as suggested in [58].

\subsubsection{Traditional replacement policies}

- Least Recently Used: The LRU evicts the least recently accessed document first, on the basis that the traffic exhibits temporal locality. Temporal locality suggests that files that have been referenced in the recent past are likely to be referenced again in the near future. Intuitively, the farther in time a document has last been requested, the less likely it will be requested in the near future. Some of the Web caching approaches such as Harvest [59] do not implement a pure LRU scheme. Harvest cache initiates the removal algorithm when the total size of cache documents exceeds a threshold value of the cache. To reduce the time that is required for executing the removal algorithm, Harvest cache sorts only a group of $\mathrm{N}$ cached documents instead all of them. Then, from this list of sorted documents, 
the least recently used $M$ files are evicted from the cache [60]. Caching policies may also use additional data structures for implementing the LRU list. For example, Squid uses a doubly liked list and a hash table index to implement the LRU list [19].

- Least Frequently Used: LFU evicts the least frequently accessed document first, on the basis that a popular document tends to have a long-term popularity profile. This policy maintains a reference count for each document in the cache. On each hit, the reference count of the requested document is incremented. On each miss if there is not sufficient space in the cache, the document with a lower frequency count is replaced. The advantage of LFU is its simple implementation, while a problem associated with it is that some documents can build up high reference counts so that they are rarely replaced, even if the documents are never requested again. This situation is referred to as cache pollution.

- LFU-Aging: To solve the problem of cache pollution, researchers proposed the concept of LFU-Aging. LFU-Aging occasionally reduces the reference counts of the documents. When the average number of reference counts of the cached files exceeds a limit, all of the reference counts are divided by two. Also, LFU-Aging limits the maximum value of the document reference counts to avoid cache pollution. [61], [62].

- Pitkow/Recker: This policy evicts objects in the LRU order, except if all the objects are accessed within the same day, in which case the largest one is removed. 


\subsubsection{Key-based replacement policies}

- Size: Size is one of the key-based cache replacement policies. This policy uses size as a key to remove the documents from the cache. If there is a tie with regard to size, size-based policies can use other policies such as the LRU or the LFU. Size-based policies are used when the goal of a cache replacement policy is to increase the hit ratio. The main draw back of this policy is that small documents are kept in the cache for long time even if they are never accessed again [63].

- LRU-MIN: The LRU-MIN policy is biased in favor of smaller objects. If there are any objects in the cache which have a size of at least S, LRU-MIN evicts the least recently used such object from the cache. If there are no objects with size being at least S, then LRU-MIN starts evicting objects in the LRU order of size being at least $\mathrm{S} / 2$. That is, the object which has the largest $\log$ (size) and is the least recently used object among all objects with the same log (size) will be evicted first [32].

- LRU-Threshold: In this policy only the documents below a certain fixed size threshold are cached. This policy uses an admission constraint to stop large documents from entering the cache. This policy achieves a high hit ratio, but performs very poorly in terms of the byte hit ratio. In this policy, it is hard to decide on an optimal threshold for a particular trace. If the threshold is too small 
there might not be many files under that size. If the threshold is too high there will too many replacements [32].

- Hyper-G: This policy is an extension of the LFU policy, where ties are broken according to the last access time. Note that under the LFU policy, ties are very likely to happen [56].

- Lowest latency First: The main goal of the previously discussed policies is to increase the hit ratio. In contrast, the lowest latency first policy [60] considers the document download time as its primary and only key (the document with the lowest download time is evicted first), so as to minimize the average download latency.

\subsubsection{Cost-based replacement policies}

We mention now the following cost-based replacement policies.

- Greedy Dual-Size (GD-Size): Cao and Irani [64] studied several proxy cache replacement policies. They introduced the Greedy Dual-Size replacement policy. GD-Size is a generalization of the Greedy Dual algorithm that was introduced by Young [65] to deal with caching of variable cost pages that have the same sizes. Since the files cached in the web have variable sizes, Cao and Irani modified the Greedy Dual algorithm to consider variable sizes as well. In GD-Size, a value $H$ is associated with each document in the cache. 
When a document is brought into the cache, the function $H=\cos t /$ size is calculated. When a replacement has to be undertaken, the document with the smallest $H$, say, min-H is removed from the cache, and every document has its $H$ value reduced by min- $H$. The associated cost is the cost of bringing a file into the cache; consequently, $H$ is the cost of bringing a file into the cache while taking into account the size of that file. The definition of the cost depends on the goal of the replacement policy. For example, if we want to increase the hit ratio, then the cost is set to unity. In this case, the GD-Size policy is refereed to as GD1. By considering the algorithm of GD-Size presented in [64], it is clear that if we use the same size files (i.e., by setting the size to 1), GD1 is identical to LRU. If the goal of the replacement policy is to minimize the average latency, then the cost is set to the downloading latency. For calculating the average latency, Cao and Irani used the method introduced in [60]. This method is the same as the one used by the HYBRID policy, presented in this Section. If the goal is to reduce network cost, the cost is set to the network cost, where the network cost can be considered as the number of packets that are transferred when retrieving the file from the server into the cache. Cao and Irani examined all of these three extensions to GD-Size policy. They showed that GD-Size outperforms existing policies in many aspects, including the hit ratio improvement, the latency reduction and the network cost reduction. They also suggested the integration of the hint-based prefetching with cache replacement policies.

- GDS-Popularity (GDSP): Jin and Bestavros generalized the GDS policy in [66]. They modified GDS by incorporating popularity information of the cached files in 
GDS. To identify the popularity of the files they defined "long term access", for each file. For each file $p$ that is in the cache, they calculated the value of $H$ as follows: $H=f(p) \times \cos t(p) / \operatorname{size}(p)$, where $f(p)$ represents the long term access frequency for the file $p$. The resulting algorithm is called the GDSPopularity or GDSP. They used the formula

$$
f_{i+1}(p)=f_{i}(p) \times 2^{-t / T}+1
$$

to estimate the long term access frequency. In this formula, $t$ is the elapsed time since the last reference to $p$, and $T$ is a constant that controls the rate of decay. For example, $T$ was set to 2 days in the simulation that was performed by them. To be able to use this formula, a popularity profile (i.e., access frequency and last access time) had to have been maintained for each file in the cache. HTTP provides the retrieval time (i.e., cost), size and the time of last access to a file. Thus, by reading this information, GDSP could compute the value of $H$, for each cached file in an online manner. Based on the value of $H$, a decision was made about the file that should be evicted. One of the problems with this method is keeping the popularity profiles in the memory for all of the cached files. They evaluated GDSP with $\cos t(p)$ set to the cost of retrieval of the file $p$ for all of the situations in which the goal of the cache was to improve the hit ratio, reduce latency and network traffic. They showed that GDSP outperforms LRU, LFU and GDS policies in many aspects.

- Hybrid: Wooster and Abrams developed the so-called Hybrid policy in [60]. The goal of their proposed algorithm was to reduce the delay that is perceived by a 
client when retrieving a file. In this algorithm, the proxy estimates the delay to retrieve a Web file from the server. Based on this estimation, the proxy assigns cost values to each cached file. The Hybrid policy evicts the files with higher retrieval cost when a replacement is necessary. To estimate the page retrieval latency, they measured the latency for stabilizing the connection between the server $_{\mathrm{i}}\left(\right.$ clat $\left._{i}\right)$ and the connection bandwidth between proxy and server $\mathrm{i}_{\mathrm{i}}$ $\left(\right.$ cbwserver $\left._{i}\right)$. The proxy calculated cbw and clat for each server when it received a document from the server.

Although the download time of the document is known to the proxy (through the HTTP request), there is no information about connection latency. The problem is that the cache runs at the application level, and does not have access to the connection latency (clat) that is accessible at the lower levels that deal with TCP and packets. Thus, an assumption is made in their algorithm to estimate the connection latency. According to this assumption, if the size of the retrieving document is less than 2 Kbytes, the download time for that document is used for estimating clat, and if the size is more than 2 Kbytes, the time is used for calculating cbw. More details about this calculation and the related algorithm are presented in [65]. They demonstrated that their Hybrid scheme works better in reducing the latency when compared to LRU and LFU.

\section{- Size-Adjusted LRU (SLRU)}

In [58], Aggarwal et al. designed a cost/size aware LRU policy called the SLRU. This policy orders the objects in terms of their ratio of cost to size, and chooses 
objects with the best cost to size ratio. In the standard LRU policy, a dynamic frequency is defined for every document that has been accessed more than once, where the number of requested documents is recorded since the last access to document $\mathrm{p}$ at time $\mathrm{k}$. The LRU policy removes objects starting from the one that has the smallest dynamic frequency, in order to optimize/reduce the cost. The standard LRU policy works as if all documents have the same size, thereby simplifying the optimization problem by ordering documents in an increasing order of their dynamic frequencies.

In the SLRU, the optimization is based on ordering documents according to their cost to size ratio, and evicting the document with the lowest ratio first to accommodate a newly arrived document. Both LRU and SLRU always cache a newly incoming document. SLRU can be extended to incorporate selective admission criteria for the newly incoming document based on the names and timestamps of last few accessed documents, the access cost, and the expiration time data. Numbers of accesses to these documents are stored in an auxiliary cache. Once a document is accessed for the first time, it is cached if there is enough space to accommodate it. If the cache is full, then the auxiliary cache is checked, and if the name of the document is not in the auxiliary cache, the document does not enter the cache and its information is added to the auxiliary cache. If the name of the document is in the auxiliary cache and the document has been accessed for at least $\mathrm{k}$ times, then it is considered as a candidate for caching according to the SLRU replacement policy. Such an admission control policy 
ensures that an incoming document is popular enough to offset the loss due to the replaced document.

To summarize, a great deal of effort has been made to maximize the hit rate. However the performance of replacement policies depends highly on the traffic characteristics of the WWW accesses. No known policy can outperform others for all Web access patterns.

\subsection{Previous Work on Web Workloads}

There are several studies reported in the literature on Web workload characteristics and Cache replacement policies as discussed in Sections 2.2 and 2.3. However, previous work related to workload characteristics did not deal explicitly with the study of the transfer size distributions of one-timer and non-one timer unique documents in detail, with either empirical or mathematical models. Also, recent studies on cache replacement policies discussed in Section 2.3 did not focus on adaptively tuning the size filter to optimize cache performance. In Sections 2.4 .1 and 2.4.2, we present an overview of related workload characteristics and cache replacement policies in the context of our analysis. These Sections also provide brief overview of how our work is different from these works reported in the literature.

\subsubsection{Web Workload Characteristics}

The research reported in [45], [46] and [47] on Web workload characteristics in the past, has focused on identifying parameters that affect caching performance. Much of the focus 
was centered on analyzing total unique documents using trace driven simulations from a variety of sources including academic, scientific and commercial environments. Their analysis has shown that the document file size follows a heavy tailed distribution, and that the WWW traffic exhibits self-similarity.

The main difference in the workload characteristics discussed in our Thesis and in the literature mentioned above, lies in the separation of unique one-timer and non-one timer documents, and in comparing them to the total unique documents in a given trace. Empirical and standard mathematical models are used to study the transfer size distribution of one-timer, non-one timer and total unique documents and also their statistical properties. These properties are helpful in better understanding the workload characteristics associated with changing traffic patterns and designing adaptive learning techniques that are vital in improving the caching performance.

\subsubsection{Adaptive Feedback admission control policy}

While several cache replacement policies have been reported in the literature, to the best of our knowledge only two policies have considered admission constraints in analyzing cache performance. These are the SLRU and LRU-Threshold policies.

SLRU designed by Aggarwal et al. [58] is a size adjusted LRU replacement policy that orders the objects in terms of their ratio of costs to sizes, and chooses objects with the best cost-to-size ratio. This policy used key admission constraints such as the names and timestamps of the last few accessed documents, and the number of accesses to these 
documents stored in an auxiliary cache. Using event and trace driven simulations, they have shown that SLRU performs better than standard LRU. However, one of the limitations of this policy is that its admission constraints are based on the past few cached documents.

Abrams et al. [32] have examined the LRU-Threshold policy to improve cache performance in terms of the document hit ratio. With the LRU scheme as the basic replacement policy, they have used a size-based threshold to avoid caching documents larger than the threshold. This policy has two limitations in terms of its cache performance: 1) The size threshold is static with relation to the cache, and 2) Even if the cache has space, a document whose size is larger than the threshold is never cached.

The main difference in the cache replacement policy discussed in our Thesis and the policies discussed above is three fold:

1. Adaptive tuning of the document size filter as an admission constraint as opposed to the static size filters used in the past studies.

2. A feedback based learning system to adaptively change the size filter based on the cache hit ratio.

3. Obtaining a balance between the documents hit ratio and the byte hit ratio.

In Chapter 3, we present the analysis and results of a study on Web workload characteristics. 


\section{One-Timer and Non-One Timer Analysis}

\subsection{Introduction}

In this Chapter we present a study of the Web workload characteristics in terms of onetimer, and non-one timer documents size distributions, and compare their performance to the total documents. This study is based on the analysis of proxy access logs collected from the National Laboratory for Applied Network Research (NLANR) [68]. A total of seven logs were obtained from five different proxy servers within NLANR. These servers are top level nodes in NLANR Web caching hierarchy. The access logs from five root caches in the US are obtained by FTP from ftp://ircache.nlanr.net/Traces/. The transfer size distribution properties of the requested one-timer, non-one timer and total documents are analyzed by examining their cumulative distribution functions (CDF) separately. Both empirical and standard mathematical models are used to study the size distribution functions.

The remainder of this Chapter is organized as follows. Section 3.2 describes in detail the data collection, data reduction and data analysis methodology for all the traces considered in this study. Statistical properties of the traces are also discussed in Section 3.2. Section 3.3 describes the mathematical models used to study the size transfer distribution of onetimer, non-one timer and total unique documents. Section 3.4 details the popularity characteristics of the workloads. A summary of the Chapter is presented in Section 3.5. 


\subsection{Data Collection, Reduction and Analysis}

\subsubsection{Data Collection}

We have used the access logs collected by NLANR for this research. NLANR manages IRCACHE, a high-level proxy cache serving other caches. In this Chapter, we consider seven different traces collected from NLANR in 2004. A variety of traces from academic, research/scientific and commercial organizations have been considered in the analysis of workload characteristics discussed in this Chapter. These traces are used in analyzing the cache performance using adaptive feedback admission control policy and will be discussed in Chapter 4.

Of the seven traces considered here, two traces are from a cache at the University of Illinois at Urbana-Champaign (UC1 and UC2), two traces from a cache at Boulder, Colorado (BO1 and BO2), one trace from a cache at University of Pittsburgh (PB), one trace from a cache at Palo Alto, California (PA) and one from a cache at New York (NY). This trace terminology will be used in the remainder of the Thesis. Each of these seven access logs captures the cache accesses made on a specific day. The duration for all these traces is for a single day ( 24 hours). Among all the logs we received upon requesting IRCACHE, we selected seven traces based on traffic volume, because higher traffic levels can provide a better understanding of the workload characteristics and the corresponding cache performance [69]. 
Table 3.1 shows the statistics of raw data obtained from all seven traces. The traces listed in the table have been shown in the descending order of the total number of document requests. The total number of raw document requests varied between 180,000 and 700,000 . The UC1 trace has the largest number of document requests, whereas the NY trace has the smallest number of requests. The total size of the document requests for all the traces varied from 1.8 Gbytes to 7.3 Gbytes. It can be seen from Table 3.1 that there is a huge variability in the number of document requests and in the total size of the requests amongst academic, research and commercial traces.

\begin{tabular}{|c|c|c|c|c|}
\hline Trace & Date & Type & $\begin{array}{c}\text { Number of } \\
\text { request }\end{array}$ & $\begin{array}{c}\text { Total size of } \\
\text { requests (MB) }\end{array}$ \\
\hline UC1 & 23-Jul-04 & Academic & 709,954 & 7358 \\
\hline UC2 & $22-J u l-04$ & Academic & 603,520 & 6361 \\
\hline BO1 & $21-J u l-04$ & Research & 432,235 & 5013 \\
\hline BO2 & $18-J u l-04$ & Research & 417,331 & 4461 \\
\hline PA & $16-J u 1-04$ & Contrercial & 353,173 & 5720 \\
\hline PB & $16-J u l-04$ & Acadernic & 294,309 & 4012 \\
\hline WY & $16-J u 1-04$ & Research & 182,320 & 1845 \\
\hline
\end{tabular}

Table 3.1: Statistics of the raw data for NLANR traces

Each entry in the access log records key attributes such as the timestamp of the request, the elapsed time of the request, the name (or IP address) of the client host making the request, the Log Tag that describes how the request was treated locally (Hit, Miss, etc), the HTTP code, the size of the document in bytes returned to the requesting client, the HTTP request method, the URL of the documents being requested, the user ident '-" for NLANR logs, Hierarchy Data/Hostname (description of how and where the requested object was fetched) and the content type. Among these key attributes, a limited set is 
used in data reduction and analysis of the access logs, as is explained in the following Section.

\subsubsection{Data Reduction}

In order to effectively use the above access logs in Table 3.1 for our analysis, it is extremely important to perform raw data reduction with a selective set of attributes discussed above [70]. The data reduction for each trace involves the following steps:

- Identifying cacheable vs non-cacheable documents. We consider a document cacheable if it did not contain substrings such as 'cgi-bin' or '?' and if it did not have a file extension such as '.cgi'. All non-cacheable documents are eliminated from the trace.

- Cacheable URLs whose origin server response code contained an appropriate HTTP GET request with a status code of 200 (OK), 206 (Partial Content) and 304 (Not Modified). A status code of 200 (OK) implies that a valid document was made available to the client, either directly from the proxy cache or by retrieving the document from another proxy cache or the origin server. A status code of 206 (Partial Content) implies a partial transfer of the document to the client in response to a partial GET request. A status code of 304 (Not Modified) implies that a client had issued a GET If-Modified-Since request to determine whether or not the client's cached copy of the document was up-to-date.

- Since each URL may have a large string format, the above cacheable URLs are converted to a unique integer value using an appropriate hash function. 
- Based on the above steps, the raw data in the access logs is reduced to clean logs containing a timestamp, the requested document size, and a unique integer representing a document URL. This data is used for further analysis of each of the traces based on the document size.

\subsubsection{Trace Statistics}

The statistical properties of the seven clean log traces obtained according to the raw data reduction process are listed in Table 3.2. The total clean log document requests for all the traces varied from $\sim 160,000$ to $\sim 610,000$, and on an average, account for $\sim 80 \%$ of the total raw documents requested, as listed in Table 3.1. UC2 had the highest percentage $(88 \%)$ whereas BO2 had the lowest percentage $(65 \%)$ of clean log documents. The total data transferred via clean log documents varied from 1.8 Gbytes (NY trace) to 6.8 Gbytes (UC1 trace).

\begin{tabular}{|c|c|c|c|c|c|c|c|}
\hline Item & $\begin{array}{c}\text { Total } \\
\text { Requests }\end{array}$ & $\begin{array}{c}\text { Total Bytes } \\
(\mathrm{MB})\end{array}$ & $\begin{array}{c}\text { Distinct } \\
\text { Requests }\end{array}$ & $\begin{array}{c}\text { Total } \\
\text { Distinct } \\
\text { Bytes (MB) }\end{array}$ & $\begin{array}{c}\text { Mean } \\
\text { (Bytes) }\end{array}$ & $\begin{array}{c}\text { Median } \\
\text { (Bytes) }\end{array}$ & $\begin{array}{c}\text { Coefficient } \\
\text { of Variation }\end{array}$ \\
\hline UC1 & 609,196 & 6865 & 296,532 & 3748 & 12637.59 & 2133 & 21.86 \\
\hline UC2 & 534,585 & 6119 & 248,096 & 3337 & 13449.85 & 1845 & 43.43 \\
\hline BO1 & 352,309 & 4613 & 229,633 & 2722 & 11853.49 & 2035 & 11.65 \\
\hline BO2 & 273,982 & 4194 & 178,946 & 2694 & 15053.64 & 1919 & 20.29 \\
\hline PA & 263,032 & 5269 & 187,722 & 3199 & 17043.09 & 2499 & 33.95 \\
\hline PB & 240,769 & 3930 & 144,658 & 1594 & 11012.52 & 1623 & 14.81 \\
\hline NY & 160,431 & 1800 & 126,214 & 1464 & 11599.22 & 2641 & 18.06 \\
\hline
\end{tabular}

Table 3.2: Statistical properties of clean log data for NLANR traces

The total number of distinct document requests for all the traces varied from $\sim 126,000$ to $\sim 296,000$ as shown in Table 3.2. On an average, the ratio of distinct to clean document requests was approximately $62 \%$, with the NY trace showing the highest percentage 
(78\%) and the UC2 trace being the lowest (46\%). The total distinct bytes transferred for these traces varied from 1.5 Gbytes (NY trace) to 3.7 Gbytes (UC1 trace). In the above data sets, the mean size of the documents transferred ranged from $\sim 11$ Kbytes to $\sim 17$ Kbytes, while the median is in the range of $\sim 1.6$ Kbytes to $\sim 3$ Kbytes. Overall, the mean and median document transfer sizes are quite small, as reported in previous studies [45]. The mean transfer size is larger than the median transfer size since there are few very large documents that skew the mean of the transfer size distribution. The coefficient of variation (COV) for each of the traces is shown in the Table 3.2 which varies from 11 to 43. The COV is obtained as the ratio of the standard deviation of the document size to its mean, and indicates that there is a high variability in the sizes of the documents transferred for all the seven traces.

\subsubsection{Data Analysis}

The data from the clean log analysis done as described above is used as an input file to a binary search tree program where the unique integer URL value is used to separate onetimer documents and unique non-one timer documents. As mentioned earlier, one-timer documents are the documents which are accessed only once during the entire trace duration, while non-one timer documents are popular documents which are accessed more than once during the trace duration considered. The separated one-timer and unique non-one timer documents are further analyzed to obtain their transfer size distribution. 


\begin{tabular}{|l|r|r|r|r|r|r|r|}
\hline Item & \multicolumn{1}{|l|}{ UC1 } & UC2 & BO1 & BO2 & PA & PB & NY \\
\hline No. of total requests & 609,196 & 534,585 & 352,309 & 273,982 & 263,032 & 240,769 & 160,431 \\
\hline No. of distinct files & 296,532 & 248,096 & 229,633 & 178,946 & 187,722 & 144,658 & 126,214 \\
\hline No. of one timer files & 255,994 & 211,176 & 206,570 & 159,494 & 169,496 & 123,099 & 115,857 \\
\hline One timer/distinct files(\%) & 86.33 & 85.12 & 89.96 & 89.13 & 90.29 & 85.10 & 91.79 \\
\hline One timer/total files(\%) & 42.02 & 39.50 & 58.63 & 58.21 & 64.44 & 51.13 & 72.22 \\
\hline Total bytes(MB) & 6864 & 6118 & 4612 & 4193 & 5268 & 3929 & 1799 \\
\hline Total distinct bytes(MB) & 3748 & 3337 & 2722 & 2694 & 3199 & 1594 & 1464 \\
\hline Total one timer bytes(MB) & 3103 & 2769 & 2296 & 2341 & 2772 & 1309 & 1336 \\
\hline One timer/total bytes(\%) & 45.21 & 45.26 & 49.78 & 55.83 & 52.62 & 33.32 & 74.26 \\
\hline One timer/distinct bytes(\%) & 82.79 & 82.98 & 84.35 & 86.90 & 86.65 & 82.12 & 91.26 \\
\hline
\end{tabular}

Table 3.3: Statistics of One-Timer documents in NLANR traces

The statistics related to one-timer documents for all the traces are listed in Table 3.3. As shown in the table, the number of requests generated for one-timer documents is in the range of $39 \%$ to $72 \%$, with an average of $55 \%$, of the total number of clean log requests for the traces discussed above. The number of one-timer documents for all traces is in the range of $85 \%$ to $92 \%$, with an average of $88 \%$, of the total number of distinct documents. The ratio of total bytes transferred for one-timer documents to total requests is $\sim 51 \%$, and one-timer documents to distinct requests is $\sim 85 \%$. This shows that the one-timer document size distribution approximately follows the number of one-timer document requests in the seven traces. The proportion of one-timer documents in NLANR traces is typically higher than that observed in the other proxy servers due to the filtering effects that exist in Web caching hierarchies [71].

In [71] Williamson analyzed the filtering effects of Web caching hierarchies. In a hierarchical cache structure, the lower level proxies send requests to higher level proxies only for the files that are not present in their cache. He reported that if lower level proxies use larger cache sizes, their requests to the higher level proxies are filtered for most of the popular documents. This means most of the requests sent to the higher level cache are for 
one-timer documents. For example, by using a trace driven simulation he measured the number of one-timer files stored in the higher level caches when lower level proxies use the cache sizes in the range of 1 to $4 \mathrm{~GB}$. The number of one-timer files stored in the higher level cache was in the range of $87 \%$ to $95 \%$ of the total number of distinct files.

Therefore, filtering effects cause large number of one-timers to be present in the NLANR traces. In spite of the large number of one-timers, approximately $8 \%$ to $15 \%$ of the documents are found to be responsible for $20 \%$ to $45 \%$ of the requests in our traces. These documents are accessed more than once and are referred to as popular files. For the NLANR traces the requests for popular files might be due to the overlap in requests from different proxies or clients.

\subsection{Transfer Size Characteristics}

This Section focuses on the transfer size distribution properties of the total clean $\log$ documents using empirical and mathematical models. Two main approaches exist for modeling distributions to a given data set: First is via empirical procedures, which are relatively easy to understand and implement and are based on intuitive and graphical properties of the distribution that we want to assess. Usually, the graphical methods are used to check and validate the assumptions because of their simplicity and speed. However, they involve a great probability of error. Second is a more formal means of assessing the underlying distribution of a data set using statistical methods. These are Goodness of Fit (GoF) distance tests, based on statistical theory. GoF distance tests are essentially based on either of two distribution elements: the cumulative distribution 
function (CDF) or the probability density function (PDF). In order to assess the underlying $\mathrm{CDF}$ assumptions for these traces, we have used the most popular Kolmogorov-Simirnov (KS) GoF statistical test due to its popularity and applicability to smaller and larger samples data set.

For all the traces considered, first the empirical CDF and probability density function (PDF) plots for total unique documents are obtained as a function of the document size, using a logarithmic scale on the horizontal axis, as shown in Figures 3.1 and 3.2 respectively. The empirical $\operatorname{CDF} F(x)$ for the data in a vector $X$ is defined as the proportion of $X$ values less than or equal to $x$. It can be seen from Figure 3.1 that almost all the documents are in the range from $\sim 100$ bytes to 100 Kbytes, with very few small transfers (below 100 bytes) and very few large transfers (above 100 Kbytes). This distribution is quite similar to the total unique document size distribution reported for Web clients [49] and Web servers [47].

Figure 3.1 indicates that $80 \%$ of the total documents requested for all the traces are below $\sim 10$ Kbytes. Documents less than $\sim 20$ Kbytes account for $\sim 90 \%$ of the total requests. The remaining $10 \%$ of the documents have sizes varying from 20 Kbytes to 275 Mbytes. It can also be seen from Figure 3.1 that for low document sizes below 10 Kbytes, there is a wider variation in the CDF amongst the different traces. As the document size increases above 10 Kbytes, the variation in CDF is insignificant indicating that the various NLANR traces have self-similarity in their document size distribution.

In order to further understand the CDF variations for lower document sizes amongst the seven different traces, the one-timer and non-one timer documents in the total distinct 
requests for each trace are separated and their transfer size characteristics are studied in detail. After this, a comparison of one-timer, non-one timer and total documents is made to bring out the impact of non-one timer variability on the total documents, and of the continued one-timer referencing dominance in the NLANR traces.

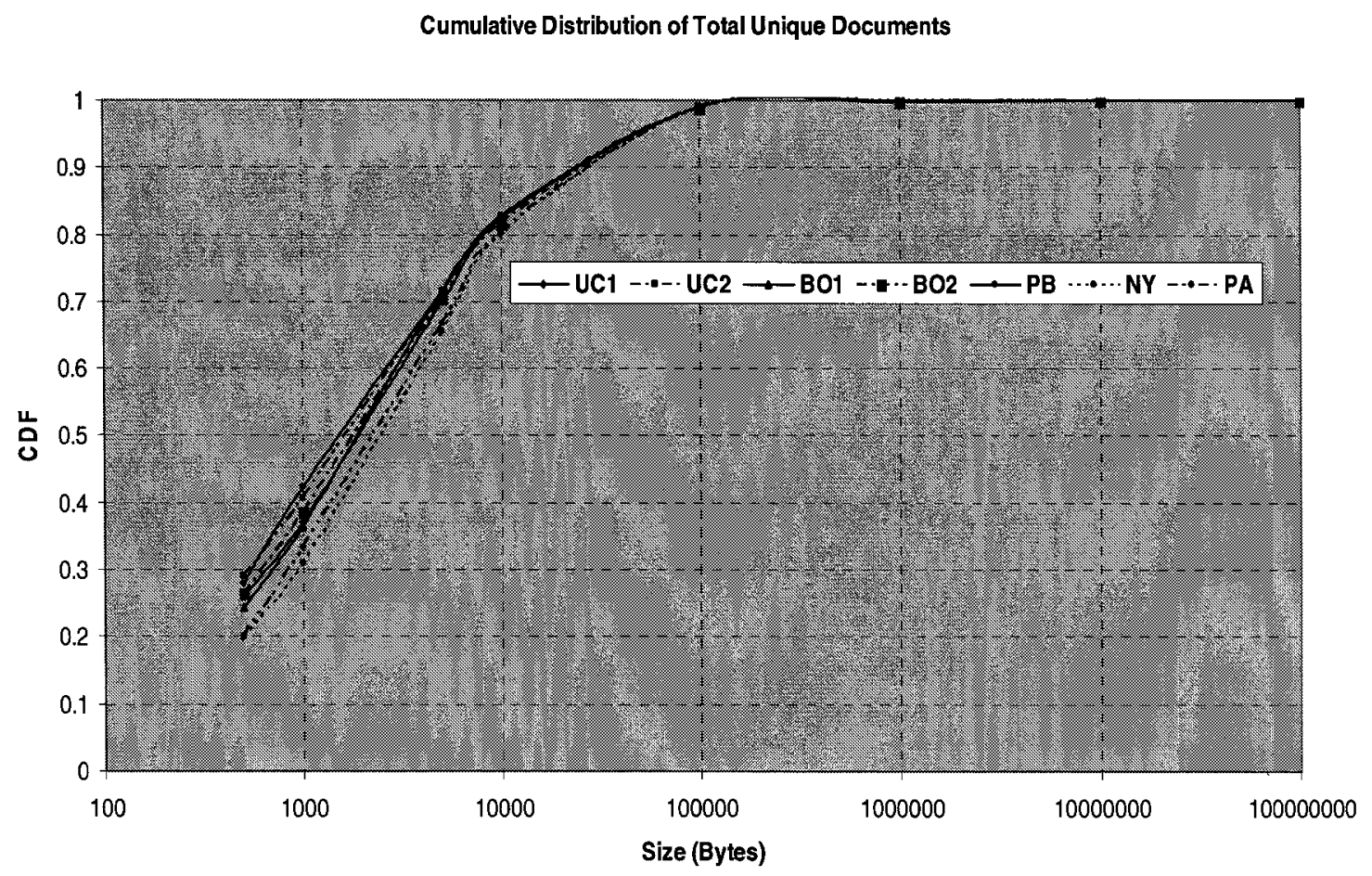

Figure 3.1: CDF of total unique documents for NLANR traces

Figure 3.2 shows the PDF plot for the total unique document requests as a function of document size. The PDF of all data sets show that their underlying distributions are skewed to the right. This observation is supported by the fact that the median is less than the mean for all seven traces as described in Table 3.2. The skewed right distributions that can be considered as potential candidates for modeling Web document sizes are the Weibull and lognormal distributions. However, we cannot assess how well they fit the 
distribution by just observing the PDF shape of the document requests. Based on the knowledge of the previous work on characterization of Web workloads, both the Weibull and lognormal distributions are used for modeling the Web documents in this Chapter.

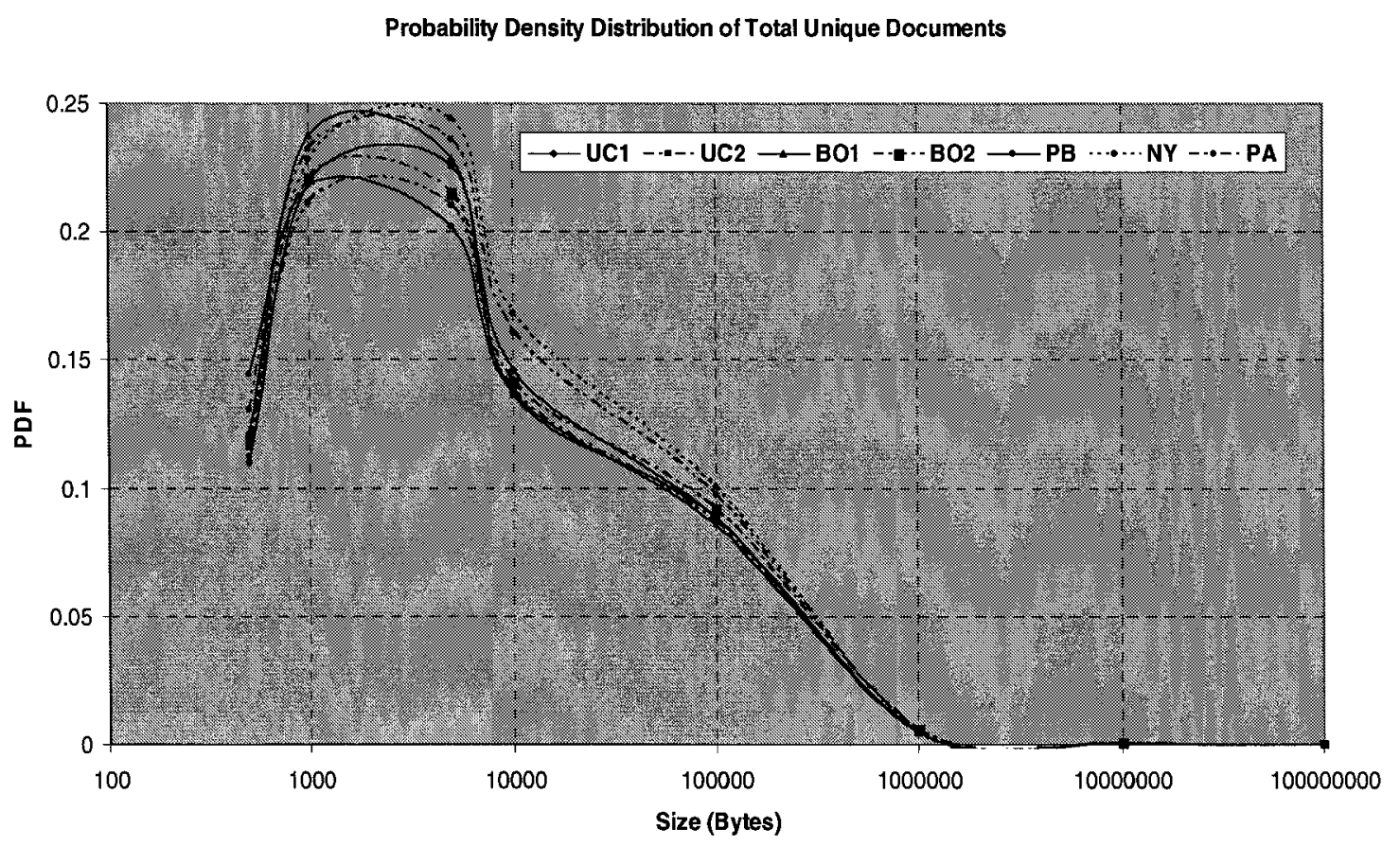

Figure 3.2: PDF of total unique documents for NLANR traces

\subsubsection{Mathematical Models}

To be able to assess how well the lognormal and Weibull models fit the empirical distributions of all the data sets, the parameters for lognormal and Weibull distribution should first be measured. There are several analytical methods to estimate the parameters of standard mathematical models and they include the Maximum Likelihood Estimator 
(MLE), the Method of Moments (MOM) and the Least Squares Method (LSM). The most popular and widely adopted maximum likelihood method is used in our analysis for estimating the parameters of the lognormal and Weibull distributions.

The theoretical CDF and PDF for lognormal distributions are as follows:

The lognormal CDF is given by

$p_{l}=F(x \mid \mu, \delta)=\frac{1}{\delta \sqrt{2 \pi}} \int_{0}^{x} \frac{e^{\frac{-(\ln (t)-\mu)^{2}}{2 \delta^{2}}}}{t} d t$

Where $\mu$ is the mean and $\delta$ is the standard deviation [72].

The lognormal PDF is given by

$$
y_{l}=f(x \mid \mu, \delta)=\frac{1}{x \delta \sqrt{2 \pi}} e^{\frac{-(\ln (x)-\mu)^{2}}{2 \delta^{2}}}
$$

The theoretical CDF and PDF for Weibull distributions are as follows:

The Weibull CDF is given by

$$
p_{w}=F(x \mid a, b)=\int_{b}^{c} b a^{-b} t^{b-1} e^{-\left(\frac{t}{a}\right)^{b}} d t=1-e^{-\left(\frac{x}{a}\right)^{b}}
$$

Where $a$ is the scale parameter and $b$ is the shape parameter [72].

The Weibull PDF is given by

$$
y_{w}=f(x \mid a, b)=b a^{-b} x^{b-1} e^{-\left(\frac{x}{a}\right)^{b}} .
$$


For the lognormal distribution, the mean and standard deviation parameters define the shape (body and tail) of the distribution function. For the Weibull distribution, the scale and shape parameters define the shape (body and tail) of the distribution function. These parameters are estimated using MLE method.

For each of the traces considered, MLE calculates the mean and standard deviation parameters of the lognormal distribution given the document size values in the data set. In a similar way, the scale and shape parameters of the Weibull distribution are obtained from the data sets. The lognormal distribution parameters are estimated using $95 \%$ confidence interval which yields the lower and upper bounds for the mean and standard deviation values. Similarly, the lower and upper bounds for scale and shape parameters of the Weibull distribution are obtained using $95 \%$ confidence interval.

\subsubsection{One-Timer and Non-one Timer Analysis}

In this Section, the total distinct documents for all the traces are separated into one-timer and non-one timer documents [2]. Empirical and fitted distributions are used to study the document referencing behavior. The one-timer document referencing as a function of the size is discussed in Section 3.3.2.1 followed by the non-one timers in Section 3.3.2.2 and total documents in Section 3.3.2.3. 


\subsubsection{One-Timer Documents}

The empirical CDF of one-timer documents along with the fitted Weibull and lognormal distributions for the seven traces is shown in Figures 3.3 to 3.9 respectively. The empirical CDF of all seven traces indicates that $80 \%$ of the requested one-timer documents size varied from 8.4 to 10.4 Kbytes. The Weibull and lognormal distribution model parameters with their $95 \%$ confidence bounds are given in Table 3.4 and Table 3.5 respectively. The confidence bounds indicate the deviation of the CDF from its mean value.

An increase in the scale parameter shifts the fitted Weibull distribution rightward, while decreasing the scale parameter shifts the curve leftward. With a fixed scale parameter, an increase in the shape parameter shifts the curve leftward, while decreasing the shape parameter shifts the curve rightward.

An increase in the $\mu$ parameter shifts the fitted lognormal distribution rightward, while decreasing the $\mu$ parameter shifts the curve leftward. With a fixed $\mu$ parameter, an increase in the $\delta$ parameter shifts the curve rightward, while decreasing the $\delta$ parameter shifts the curve leftward.

For all the traces considered, Figures 3.3 to 3.9 visually indicate that the fitted CDF's are closer to the empirical CDF. Also, the one-timer documents size variation in these traces exhibit self-similarity. 


\begin{tabular}{|c|c|c|c|c|c|c|}
\hline & \multicolumn{3}{|c|}{ Scale Parameter } & \multicolumn{3}{c|}{ Shape Parameter } \\
\cline { 2 - 7 } TRACE & Value & $\begin{array}{c}\text { Lower } \\
\text { bound }\end{array}$ & $\begin{array}{c}\text { Upper } \\
\text { bound }\end{array}$ & Value & $\begin{array}{c}\text { Low er } \\
\text { bound }\end{array}$ & $\begin{array}{c}\text { Upper } \\
\text { bound }\end{array}$ \\
\hline UC1 & 5241.36 & 5204.67 & 5278.29 & 0.5847 & 0.5832 & 0.5862 \\
\hline UC2 & 5005.27 & 4965.45 & 5045.42 & 0.5662 & 0.5646 & 0.5678 \\
\hline BO1 & 5114.33 & 5074.24 & 5154.74 & 0.5816 & 0.5799 & 0.5832 \\
\hline BO2 & 5232.75 & 5183.45 & 5282.53 & 0.5501 & 0.5484 & 0.5519 \\
\hline PA & 5961.79 & 5909.42 & 6014.64 & 0.5718 & 0.5700 & 0.5735 \\
\hline PB & 4640.49 & 4592.55 & 4688.93 & 0.5709 & 0.5687 & 0.5730 \\
\hline NY & 6003.12 & 5943.84 & 6062.98 & 0.6147 & 0.6123 & 0.6171 \\
\hline
\end{tabular}

Table 3.4: Weibull fitted parameters for One-Timer CDF

\begin{tabular}{|c|c|c|c|c|c|c|}
\hline & \multicolumn{3}{|c|}{ Mean Parameter } & \multicolumn{3}{c|}{ Standard Deviation Parameter } \\
\cline { 2 - 7 } TRACE & Value & $\begin{array}{c}\text { Low er } \\
\text { bound }\end{array}$ & $\begin{array}{c}\text { Upper } \\
\text { bound }\end{array}$ & Value & $\begin{array}{c}\text { Low er } \\
\text { bound }\end{array}$ & $\begin{array}{c}\text { Upper } \\
\text { bound }\end{array}$ \\
\hline UC1 & 7.7702 & 7.7642 & 7.7762 & 1.5583 & 1.5540 & 1.5626 \\
\hline UC2 & 7.6967 & 7.6898 & 7.7035 & 1.6024 & 1.5975 & 1.6072 \\
\hline BO1 & 7.7399 & 7.7332 & 7.7466 & 1.5523 & 1.5475 & 1.5570 \\
\hline BO2 & 7.7306 & 7.7227 & 7.7385 & 1.6102 & 1.6046 & 1.6158 \\
\hline PA & 7.9049 & 7.8976 & 7.9123 & 1.5457 & 1.5405 & 1.5509 \\
\hline PB & 7.6177 & 7.6088 & 7.6267 & 1.5988 & 1.5925 & 1.6051 \\
\hline NY & 7.9249 & 7.9160 & 7.9338 & 1.5426 & 1.5364 & 1.5489 \\
\hline
\end{tabular}

Table 3.5: Lognormal fitted parameters for One-Timer CDF

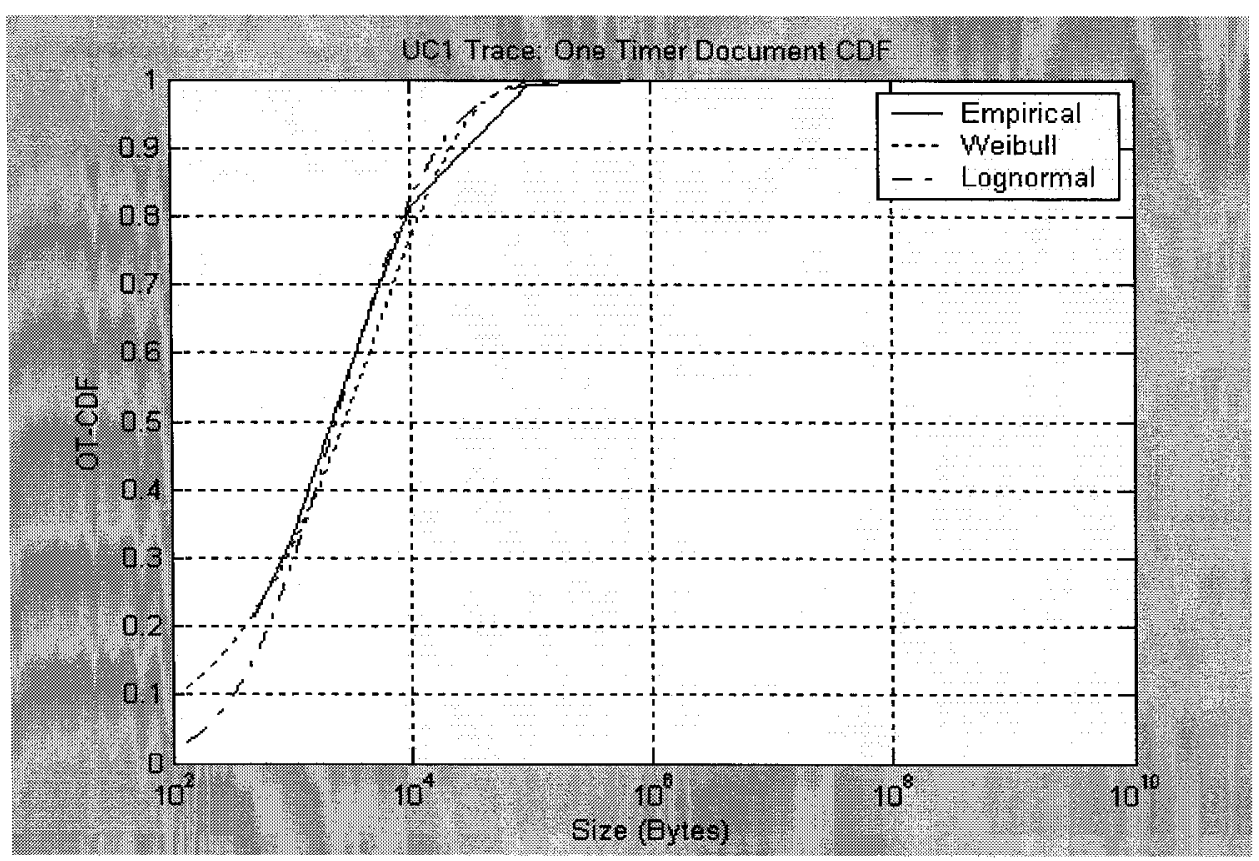

Figure 3.3: CDF of One-Timer documents for UC1 trace 


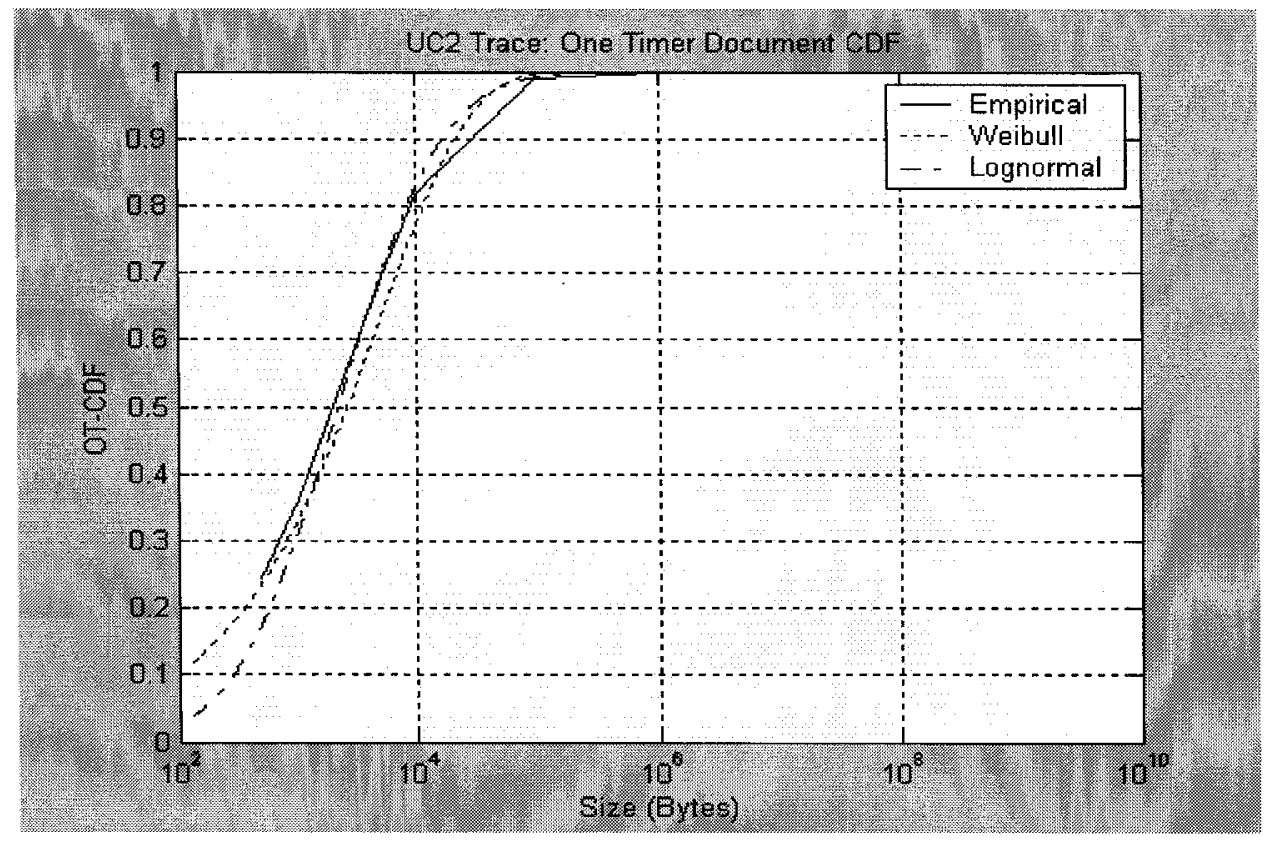

Figure 3.4: CDF of One-Timer documents for UC2 trace

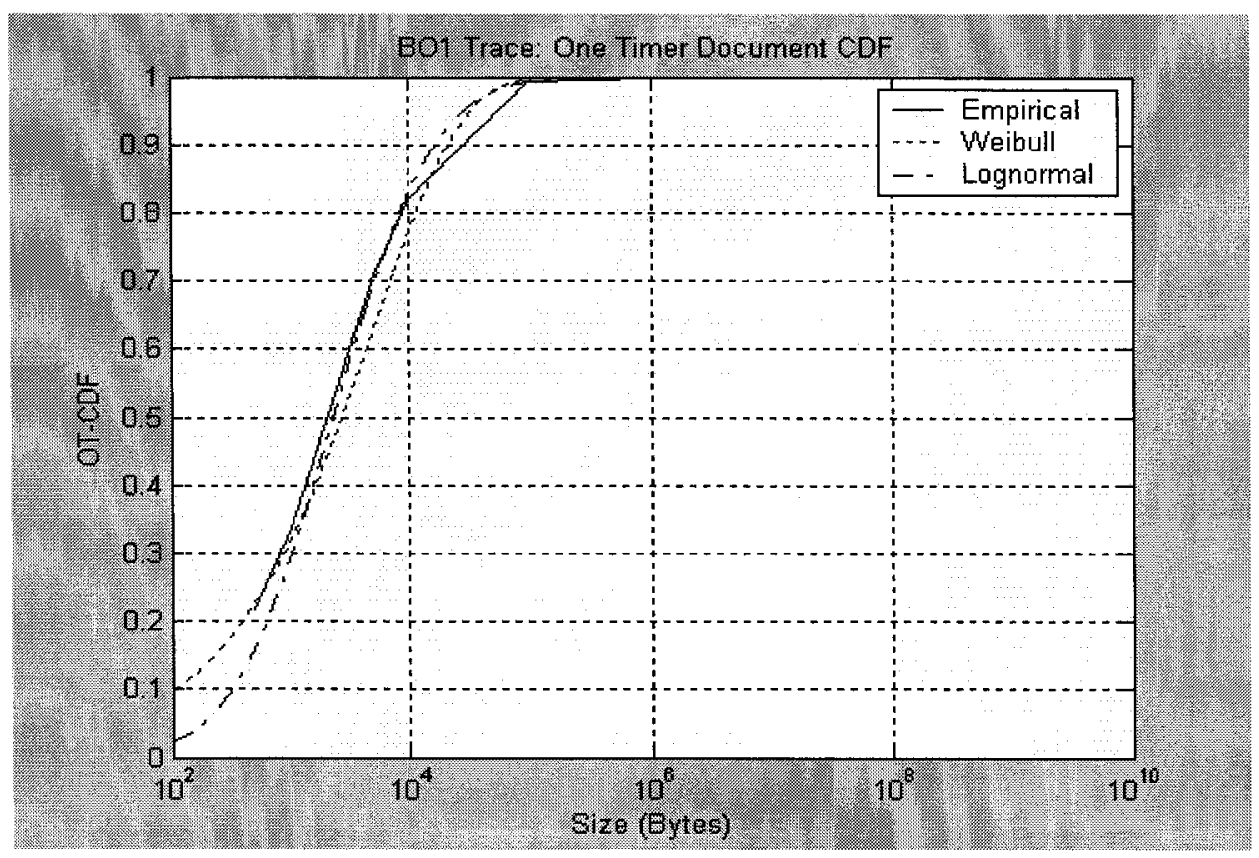

Figure 3.5: CDF of One-Timer documents for BO1 trace 


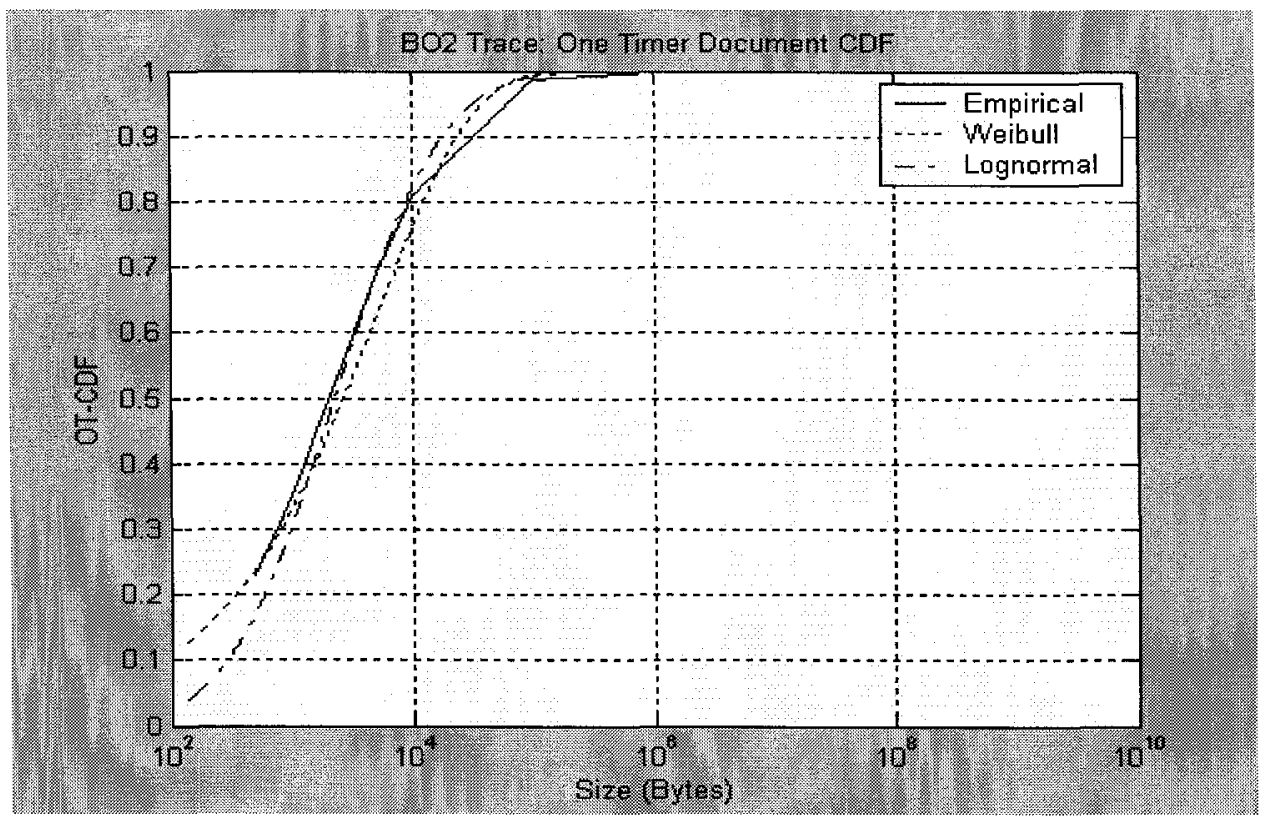

Figure 3.6: $\mathrm{CDF}$ of One-Timer documents for $\mathrm{BO} 2$ trace

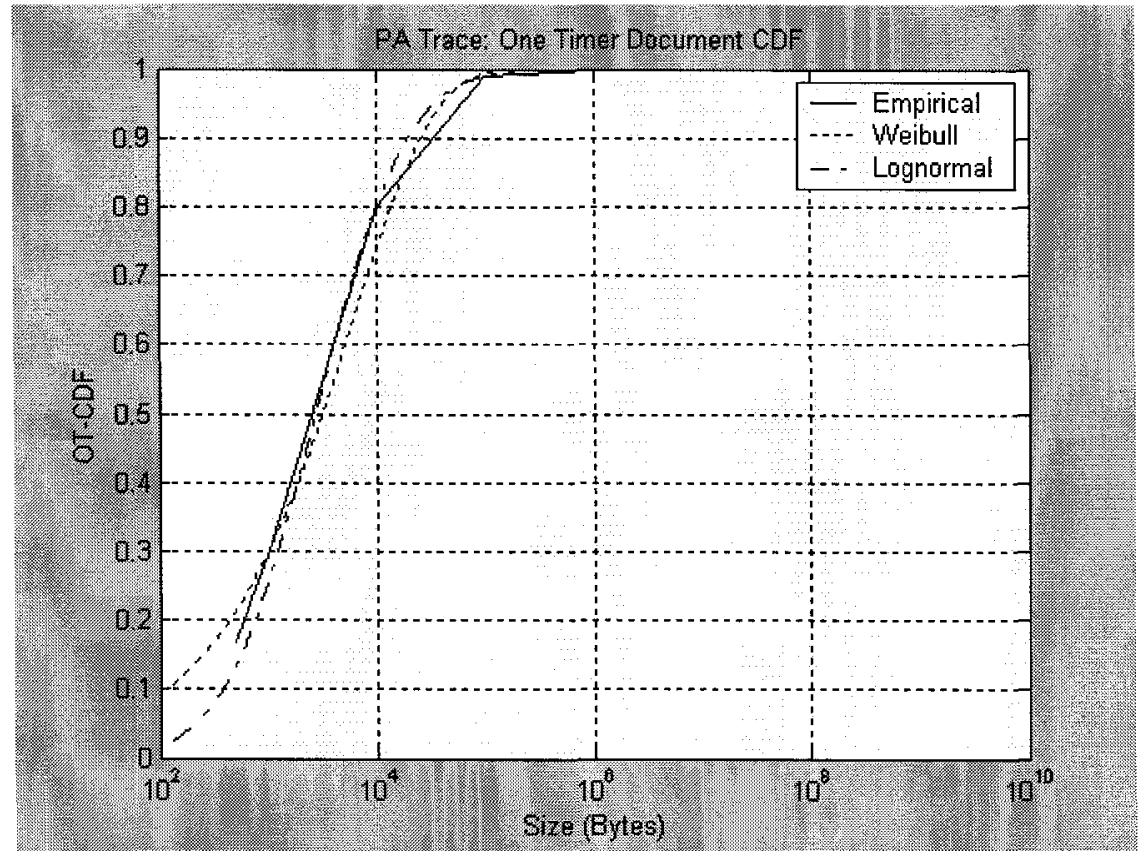

Figure 3.7: CDF of One-Timer documents for PA trace 


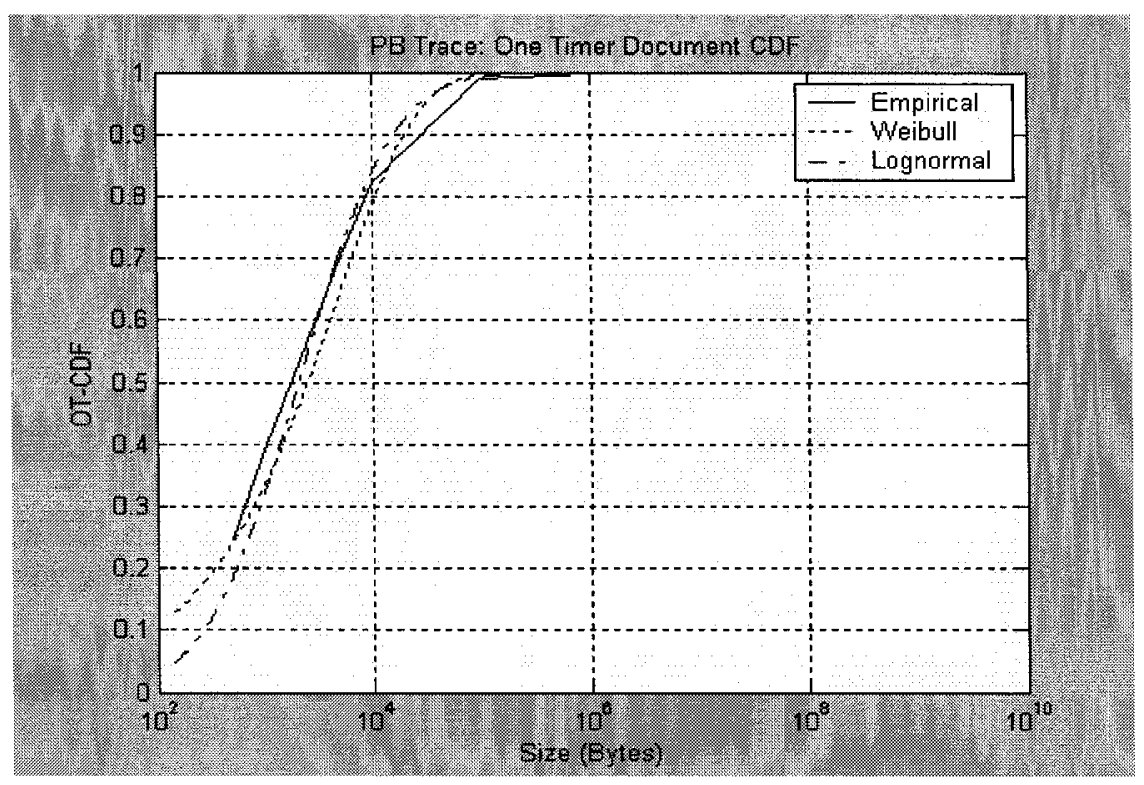

Figure 3.8: CDF of One-Timer documents for PB trace

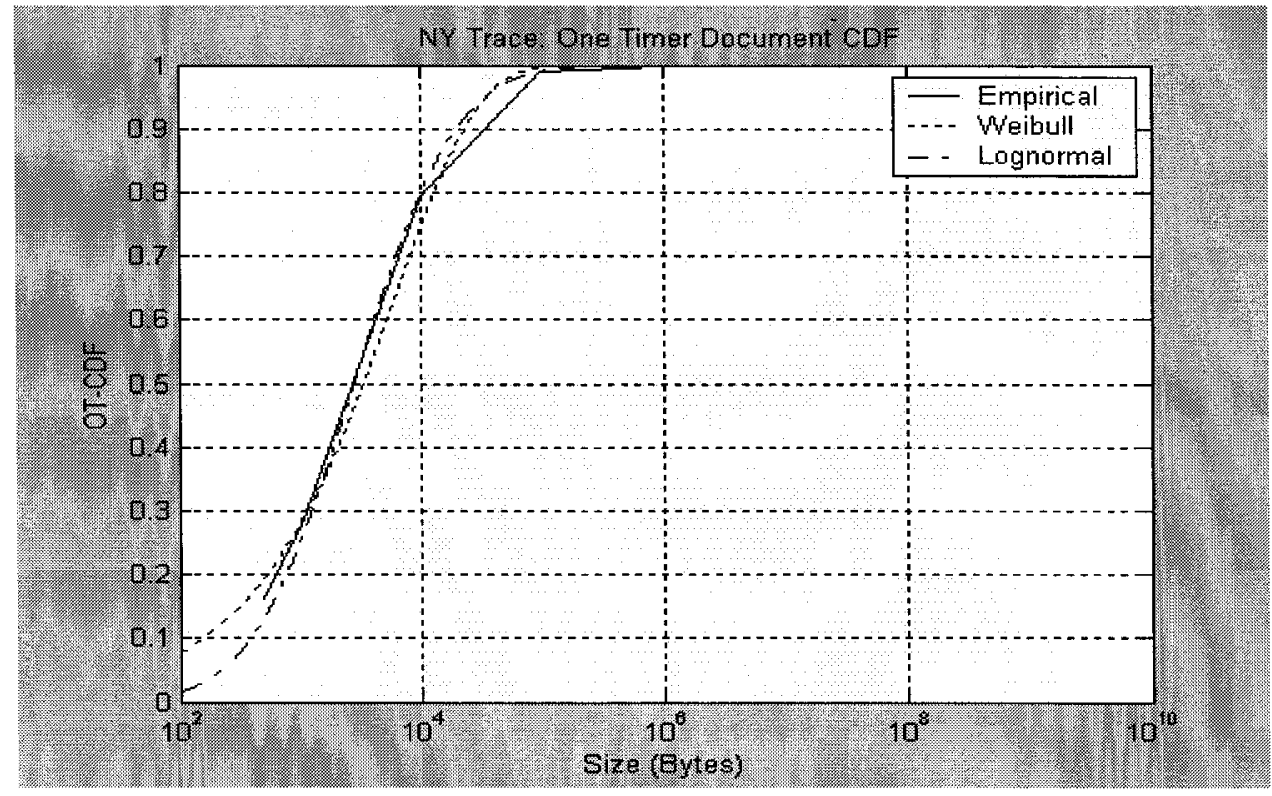

Figure 3.9: CDF of One-Timer documents for NY trace 


\subsubsection{Non-One Timer Documents}

The empirical CDF of non-one timer documents along with the fitted Weibull and lognormal distributions for the seven traces are shown in Figures 3.10 to 3.16 respectively. The empirical CDF of the seven traces indicates that $80 \%$ of the unique non-one timer documents size varied from 4 to 6 Kbytes. The Weibull and lognormal fitted model parameters for the non-one timer documents in the seven traces are given in Table 3.6 and Table 3.7. For all the traces considered, Figures 3.10 to 3.16 visually indicate that the fitted CDF's are closer to the empirical CDF and that the distribution parameters are quite similar to each other, indicating, as before, the self-similarity in the non-one timer documents distribution.

\begin{tabular}{|c|c|c|c|c|c|c|}
\hline \multirow{2}{*}{ TRACE } & \multicolumn{3}{|c|}{ Scale Parameter } & \multicolumn{3}{c|}{ Shape Parameter } \\
\cline { 2 - 7 } & Value & $\begin{array}{c}\text { Low er } \\
\text { bound }\end{array}$ & $\begin{array}{c}\text { Upper } \\
\text { bound }\end{array}$ & Value & $\begin{array}{c}\text { Low er } \\
\text { bound }\end{array}$ & $\begin{array}{c}\text { Upper } \\
\text { bound }\end{array}$ \\
\hline UC1 & 2642.73 & 2582.95 & 2703.88 & 0.4531 & 0.4503 & 0.4560 \\
\hline UC2 & 2454.07 & 2396.26 & 2513.27 & 0.4557 & 0.4527 & 0.4587 \\
\hline BO1 & 3313.18 & 3215.07 & 3414.30 & 0.4569 & 0.4531 & 0.4608 \\
\hline BO2 & 2650.93 & 2564.54 & 2740.23 & 0.4516 & 0.4475 & 0.4557 \\
\hline PA & 3065.78 & 2960.84 & 3174.43 & 0.4435 & 0.4393 & 0.4477 \\
\hline PB & 3203.09 & 3109.40 & 3299.59 & 0.4785 & 0.4743 & 0.4828 \\
\hline NY & 2269.98 & 2168.22 & 2376.54 & 0.4471 & 0.4414 & 0.4529 \\
\hline
\end{tabular}

Table 3.6: Weibull fitted parameters for Non-one Timer CDF

\begin{tabular}{|c|c|c|c|c|c|c|}
\hline \multirow{2}{*}{ TRACE } & \multicolumn{3}{|c|}{ Mean Parameter } & \multicolumn{3}{c|}{ Standard Deviation Parameter } \\
\cline { 2 - 7 } & Value & $\begin{array}{c}\text { Lower } \\
\text { bound }\end{array}$ & $\begin{array}{c}\text { Upper } \\
\text { bound }\end{array}$ & Value & $\begin{array}{c}\text { Lower } \\
\text { bound }\end{array}$ & $\begin{array}{c}\text { Upper } \\
\text { bound }\end{array}$ \\
\hline UC1 & 6.9271 & 6.9103 & 6.9439 & 1.7255 & 1.7137 & 1.7374 \\
\hline UC2 & 6.8687 & 6.8514 & 6.8860 & 1.6919 & 1.6797 & 1.7042 \\
\hline BO1 & 7.1368 & 7.1138 & 7.1598 & 1.7815 & 1.7654 & 1.7979 \\
\hline BO2 & 6.9315 & 6.9073 & 6.9557 & 1.7233 & 1.7063 & 1.7406 \\
\hline PA & 7.0335 & 7.0070 & 7.0601 & 1.8298 & 1.8112 & 1.8488 \\
\hline PB & 7.1264 & 7.1030 & 7.1498 & 1.7530 & 1.7366 & 1.7697 \\
\hline NY & 6.7202 & 6.6848 & 6.7556 & 1.8386 & 1.8139 & 1.8640 \\
\hline
\end{tabular}

Table 3.7: Lognormal fitted parameters for Non-one Timer CDF 


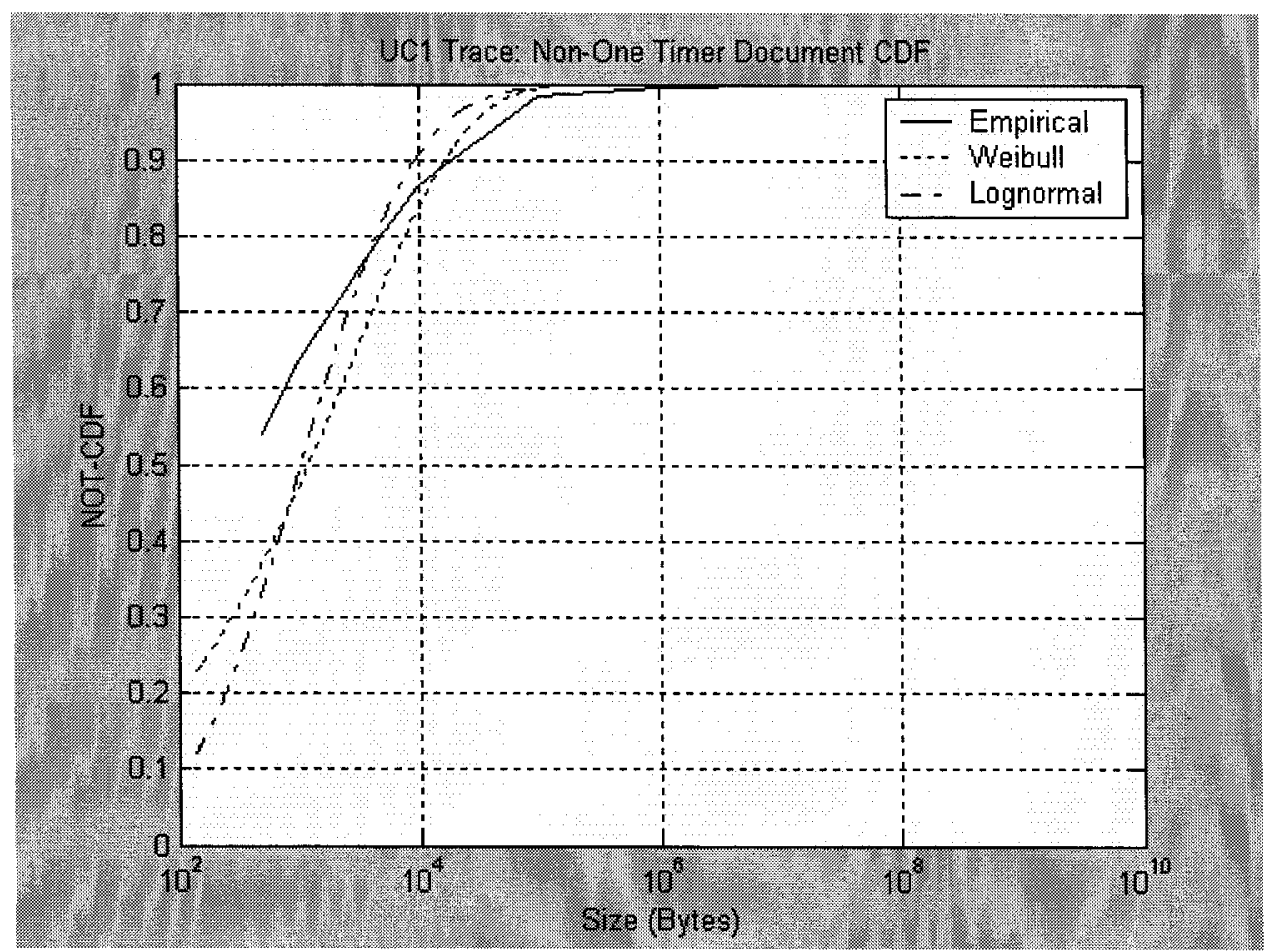

Figure 3.10: CDF of Non-one Timer documents for UC1 trace

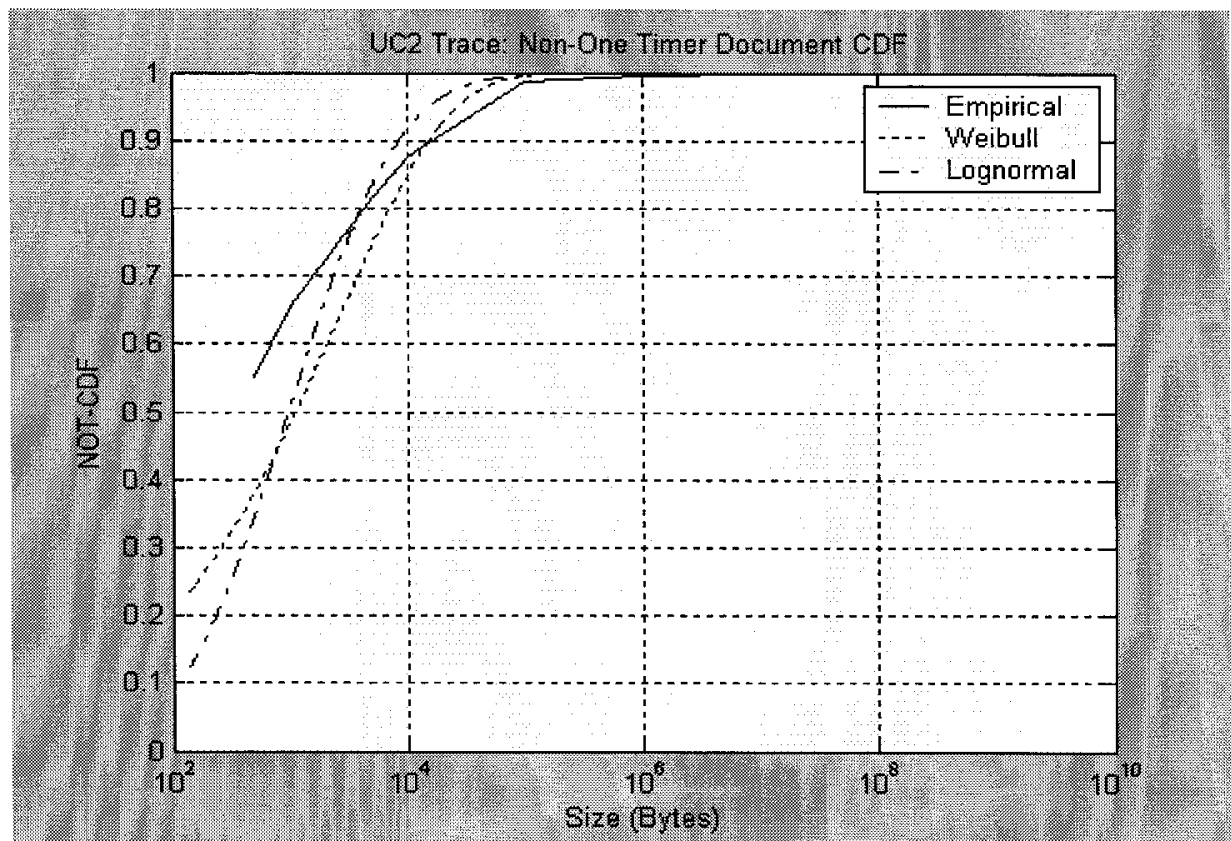

Figure 3.11: CDF of Non-one Timer documents for UC2 trace 


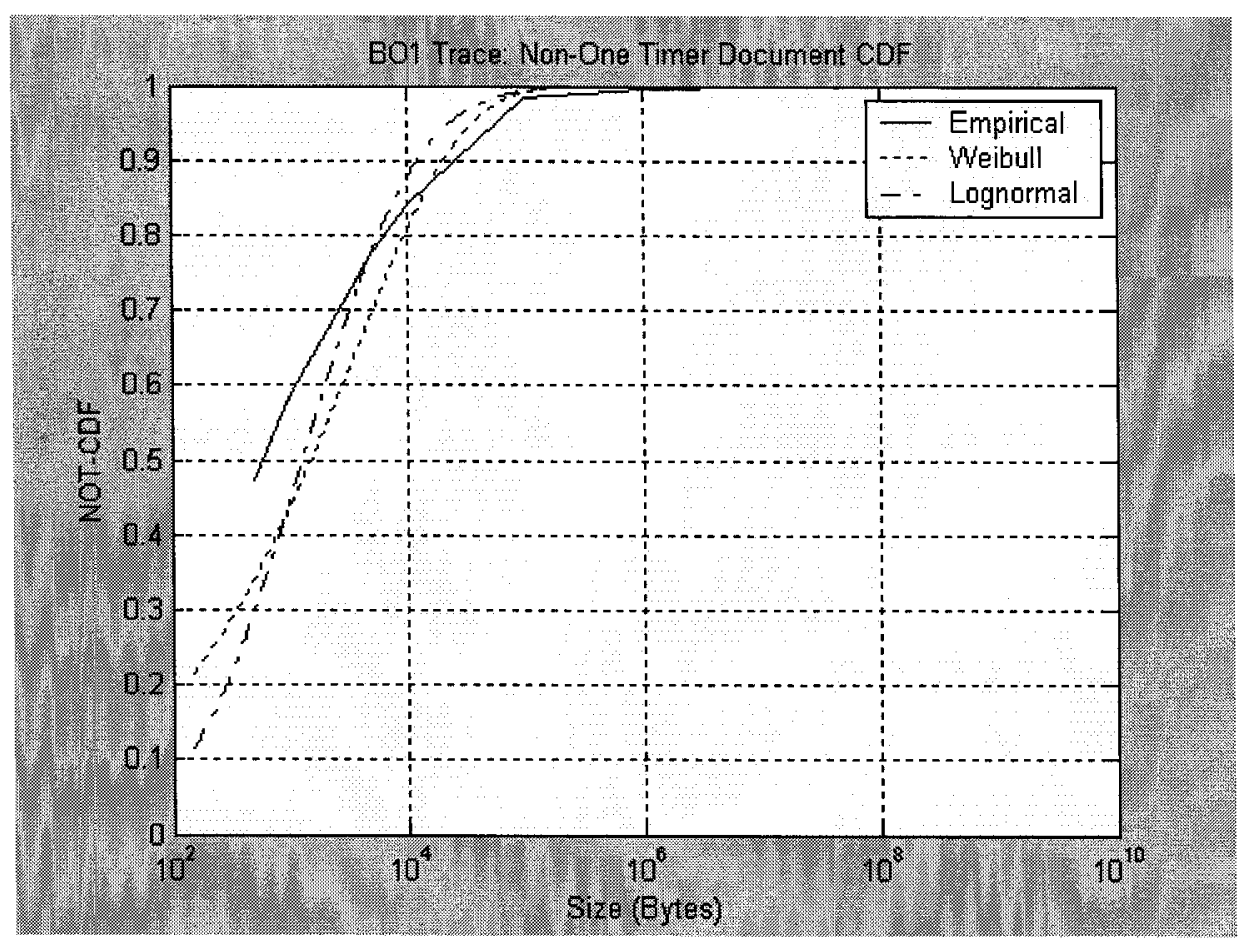

Figure 3.12: CDF of Non-one Timer documents for BO1 trace

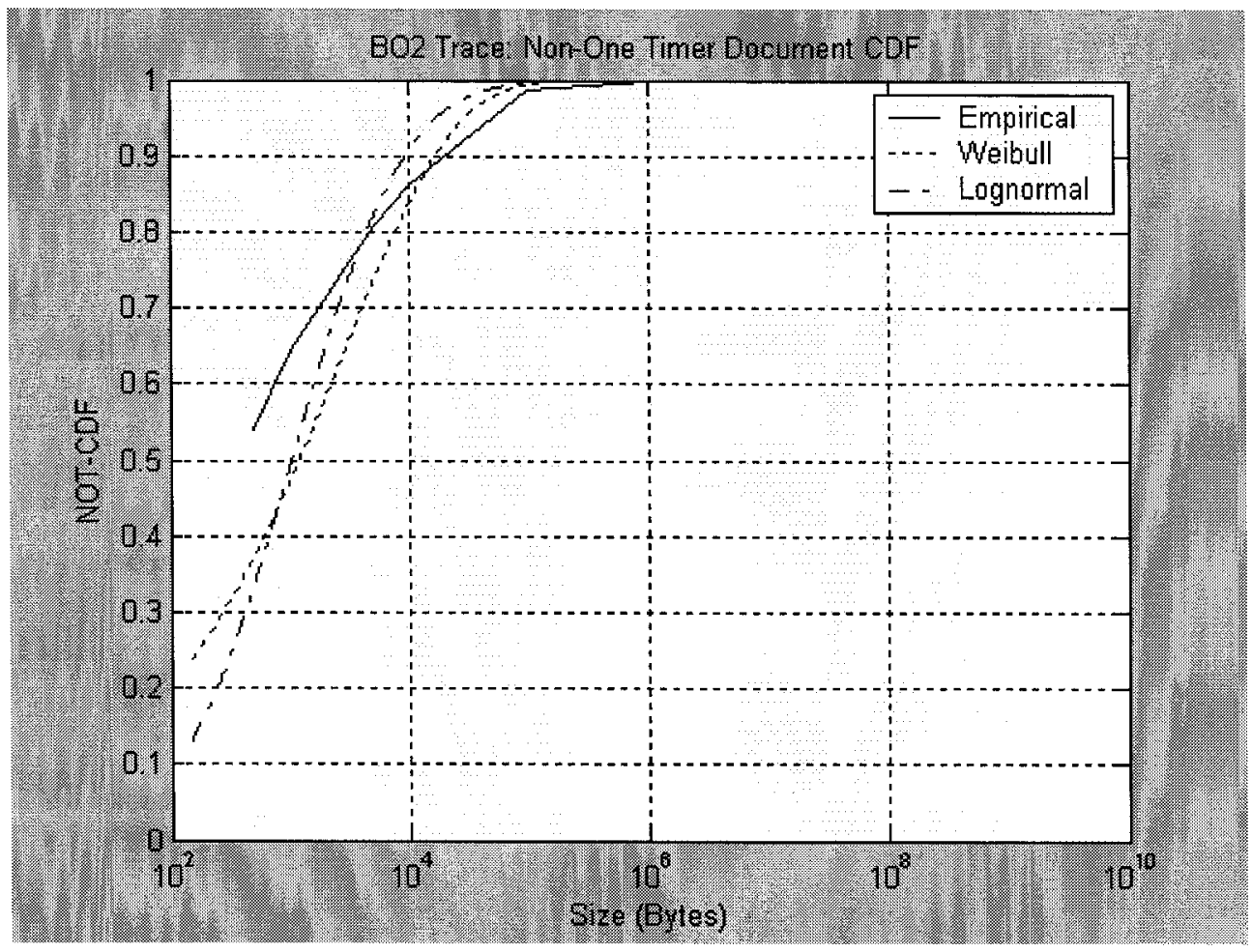

Figure 3.13: CDF of Non-one Timer documents for $\mathrm{BO} 2$ trace 


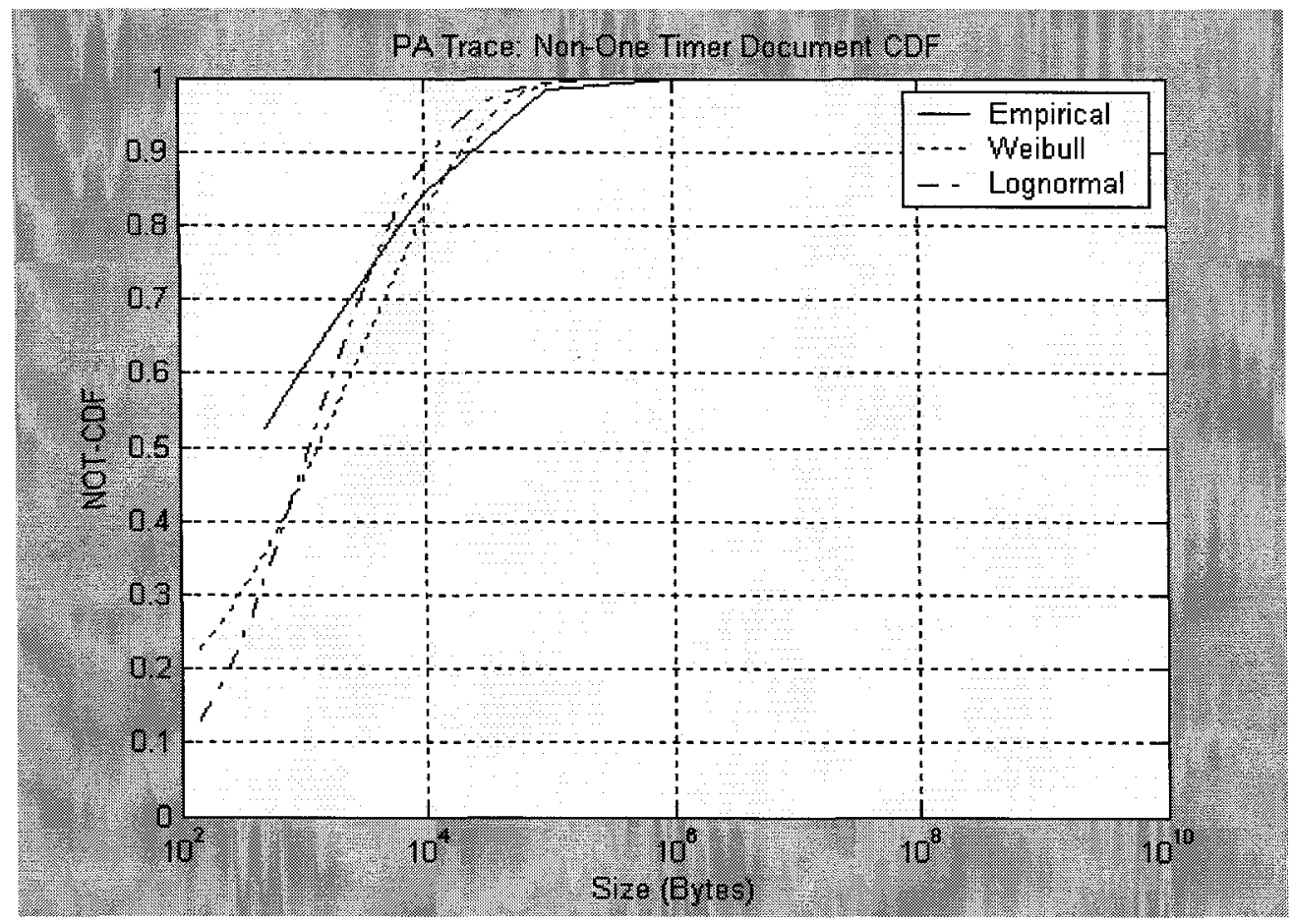

Figure 3.14: CDF of Non-one Timer documents for PA trace

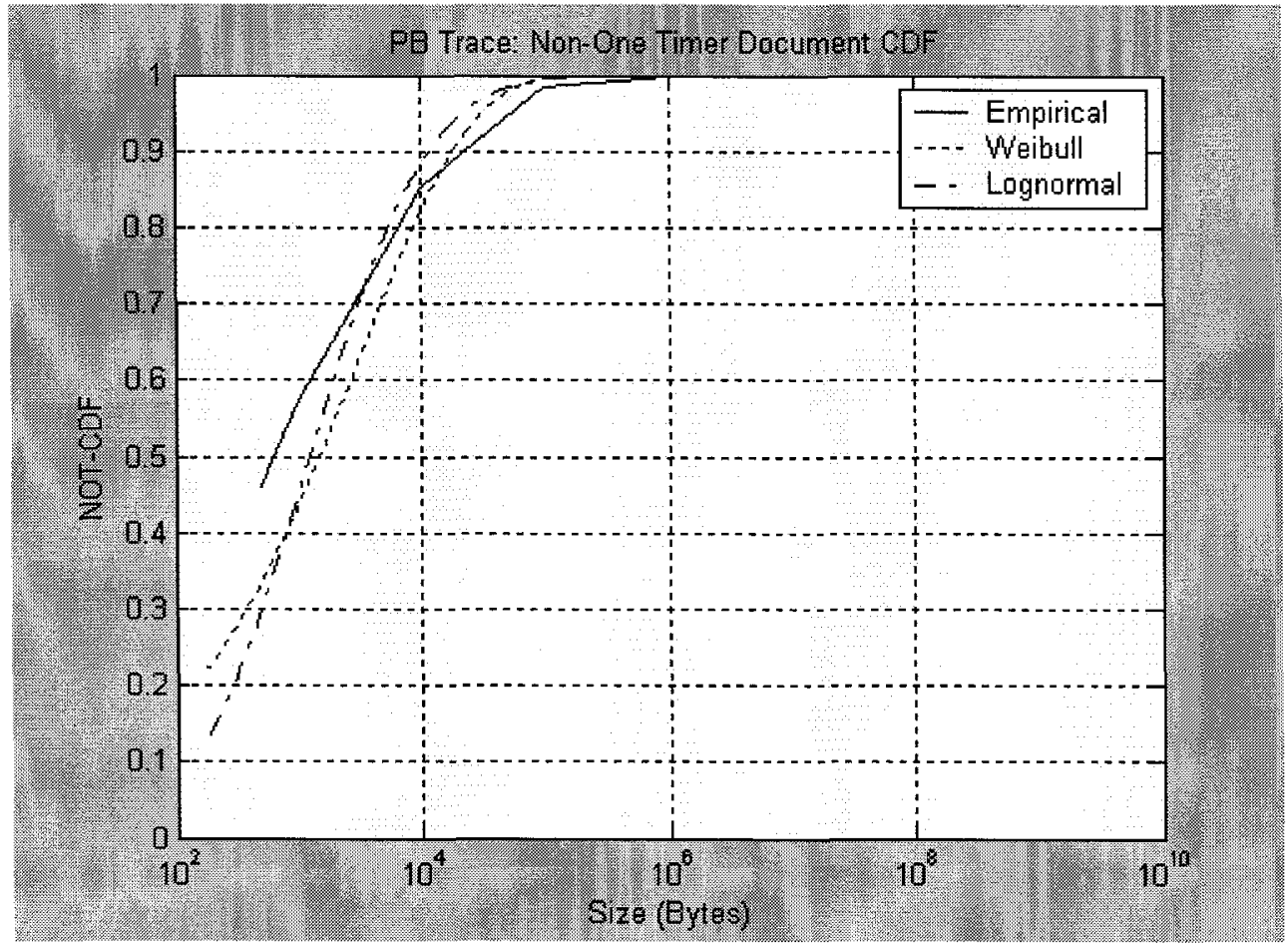

Figure 3.15: CDF of Non-one Timer documents for PB trace 


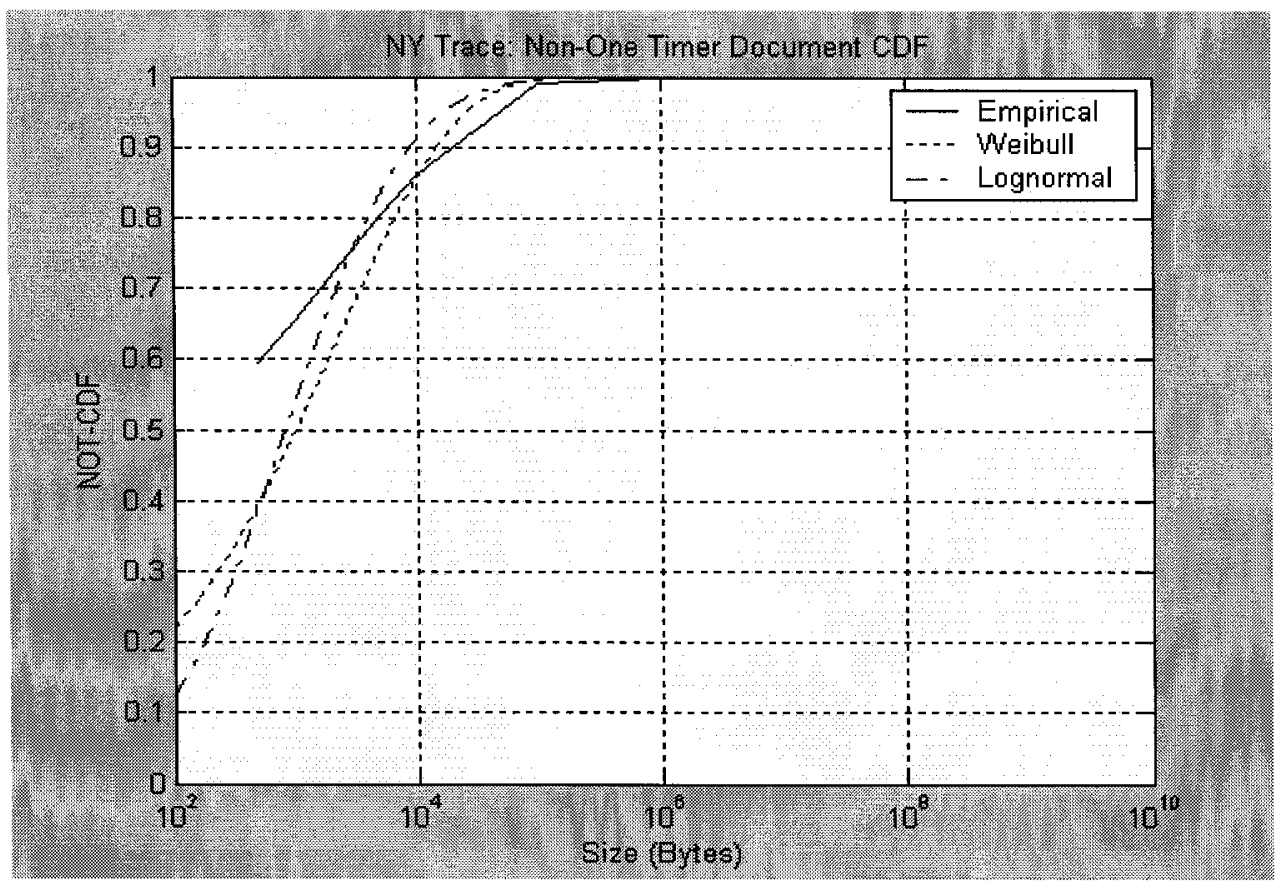

Figure 3.16: CDF of Non-one Timer documents for NY trace

\subsubsection{Total Documents}

The empirical CDF of combined one-timer and non-one timer documents along with the fitted Weibull and lognormal distributions are shown in Figures 3.17 to 3.23 respectively. The CDF of the seven traces indicates that $80 \%$ of the requested documents are less than 10 Kbytes. The Weibull and lognormal fitted model parameters for the combined onetimer and non-one timer documents in the seven traces are given in Table 3.8 and Table 3.9. For all the traces considered, Figures 3.17 to 3.23 visually indicate that the fitted CDF's match the empirical CDF, and that the distribution parameters are quite similar to each other. Again, this indicates indicating self-similarity in the combined one-timer and non-one timer documents distribution. 


\begin{tabular}{|c|c|c|c|c|c|c|}
\hline \multirow{2}{*}{ TRACE } & \multicolumn{3}{|c|}{ Scale Parameter } & \multicolumn{3}{c|}{ Shape Parameter } \\
\cline { 2 - 7 } & Value & $\begin{array}{l}\text { Low er } \\
\text { bound }\end{array}$ & $\begin{array}{c}\text { Upper } \\
\text { bound }\end{array}$ & Value & $\begin{array}{l}\text { Low er } \\
\text { bound }\end{array}$ & $\begin{array}{c}\text { Upper } \\
\text { bound }\end{array}$ \\
\hline UC1 & 4828.32 & 4795.46 & 4861.41 & 0.5591 & 0.5578 & 0.5604 \\
\hline UC2 & 4554.26 & 4519.41 & 4589.39 & 0.5435 & 0.5420 & 0.5449 \\
\hline BO1 & 4918.03 & 4880.28 & 4956.08 & 0.5634 & 0.5619 & 0.5649 \\
\hline BO2 & 4898.06 & 4853.29 & 4943.25 & 0.5356 & 0.5340 & 0.5372 \\
\hline PA & 5636.91 & 5588.37 & 5685.87 & 0.5545 & 0.5529 & 0.5561 \\
\hline PB & 4409.83 & 4366.45 & 4453.63 & 0.5535 & 0.5515 & 0.5554 \\
\hline NY & 5619.21 & 5564.16 & 5674.80 & 0.5938 & 0.5916 & 0.5961 \\
\hline
\end{tabular}

Table 3.8: Weibull fitted parameters for Total documents CDF

\begin{tabular}{|c|c|c|c|c|c|c|}
\hline \multirow{2}{*}{ TRACE } & \multicolumn{4}{|c|}{ Mean Parameter } & \multicolumn{3}{|c|}{ Standard Deviation Parameter } \\
\cline { 2 - 7 } & Value & $\begin{array}{l}\text { Low er } \\
\text { bound }\end{array}$ & $\begin{array}{c}\text { Upper } \\
\text { bound }\end{array}$ & Value & $\begin{array}{c}\text { Low er } \\
\text { bound }\end{array}$ & $\begin{array}{c}\text { Upper } \\
\text { bound }\end{array}$ \\
\hline UC1 & 7.6549 & 7.6492 & 7.6607 & 1.6085 & 1.6044 & 1.6126 \\
\hline UC2 & 7.5735 & 7.5670 & 7.5799 & 1.6426 & 1.6381 & 1.6472 \\
\hline BO1 & 7.6793 & 7.6728 & 7.6858 & 1.5872 & 1.5826 & 1.5918 \\
\hline BO2 & 7.6437 & 7.6361 & 7.6513 & 1.6418 & 1.6364 & 1.6472 \\
\hline PA & 7.8203 & 7.8131 & 7.8275 & 1.5965 & 1.5914 & 1.6016 \\
\hline PB & 7.5445 & 7.5361 & 7.5529 & 1.6321 & 1.6262 & 1.6381 \\
\hline NY & 7.8260 & 7.8172 & 7.8349 & 1.6035 & 1.5972 & 1.6097 \\
\hline
\end{tabular}

Table 3.9: Lognormal fitted parameters for Total documents CDF 


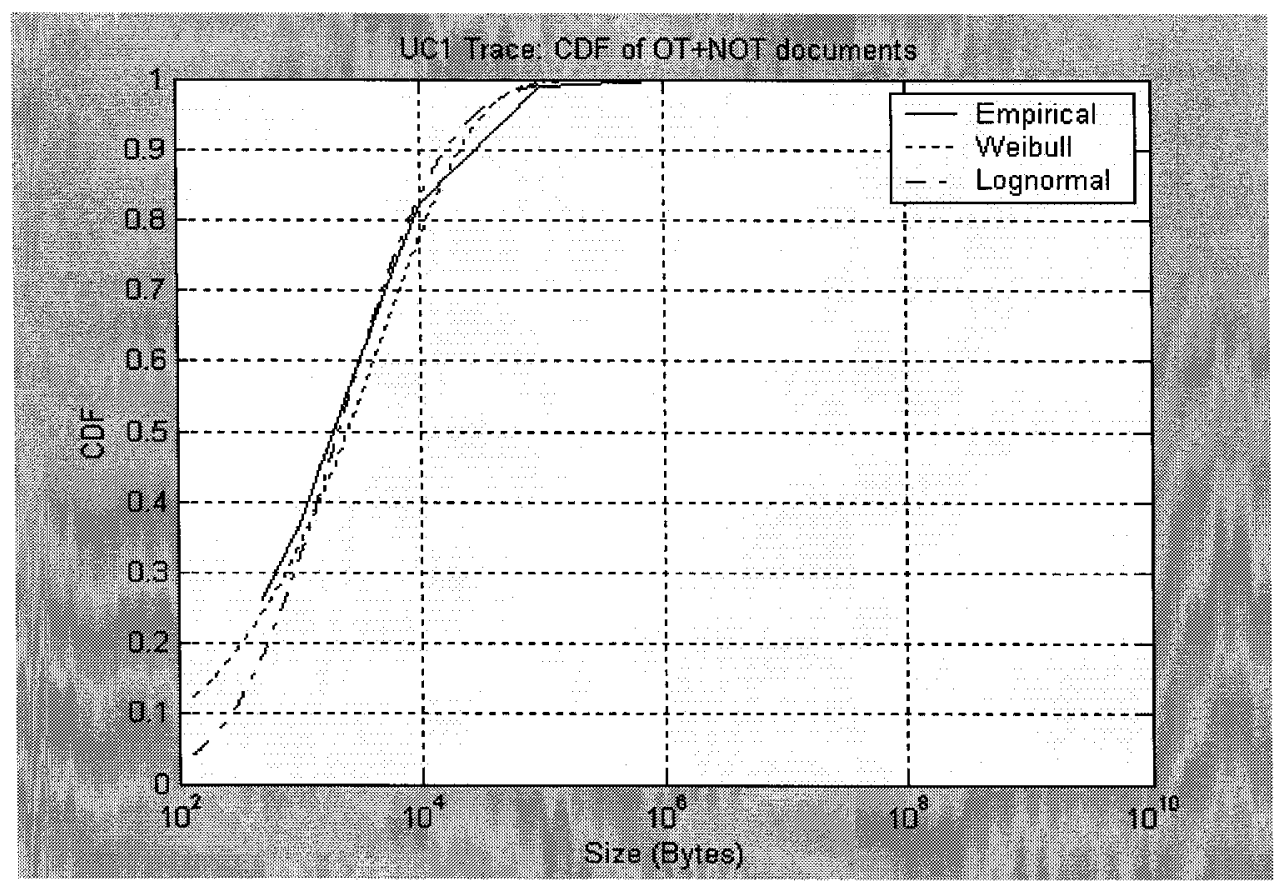

Figure 3.17: CDF of Total documents for UC1 trace

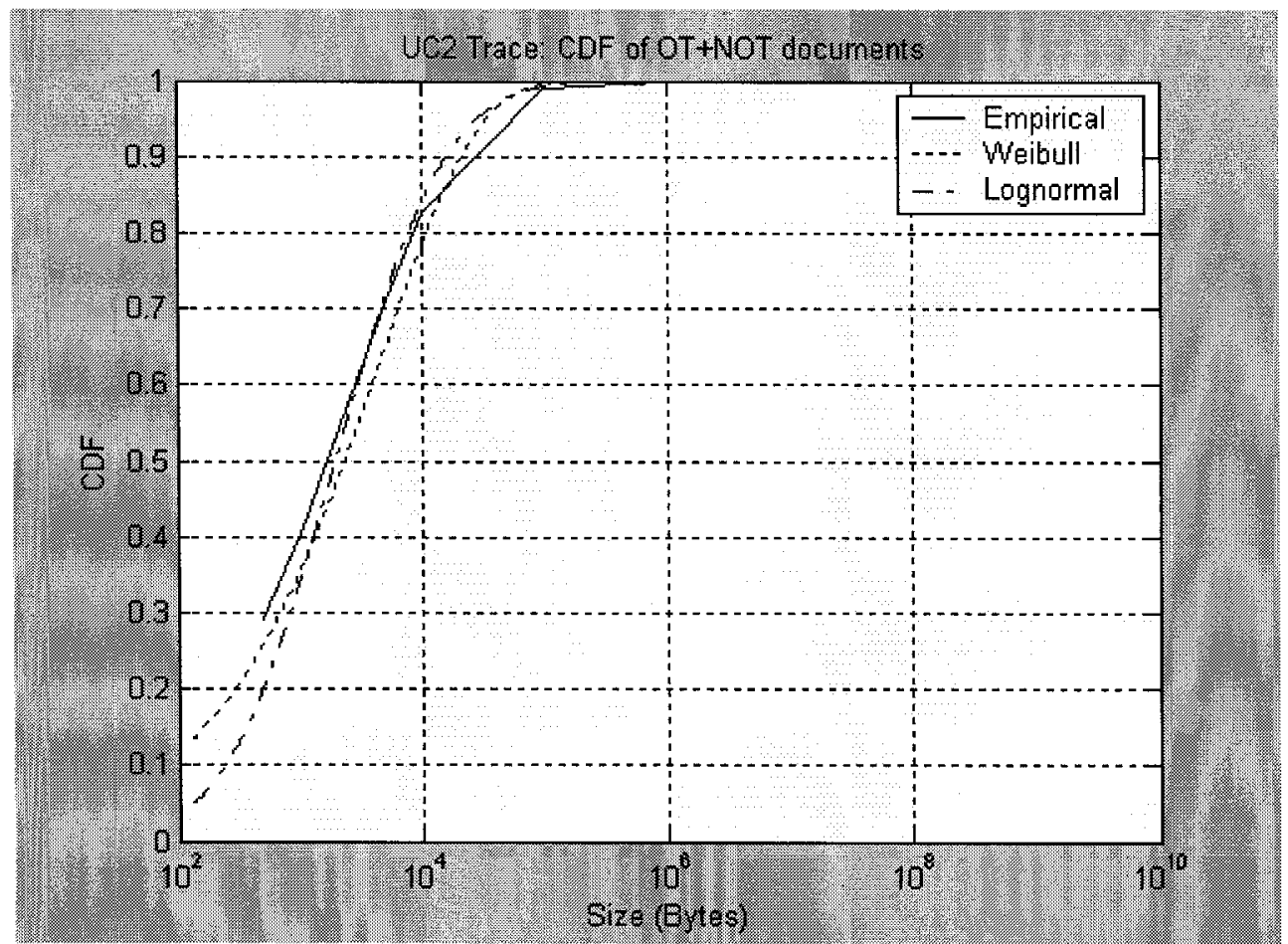

Figure 3.18: CDF of Total documents for UC2 trace 


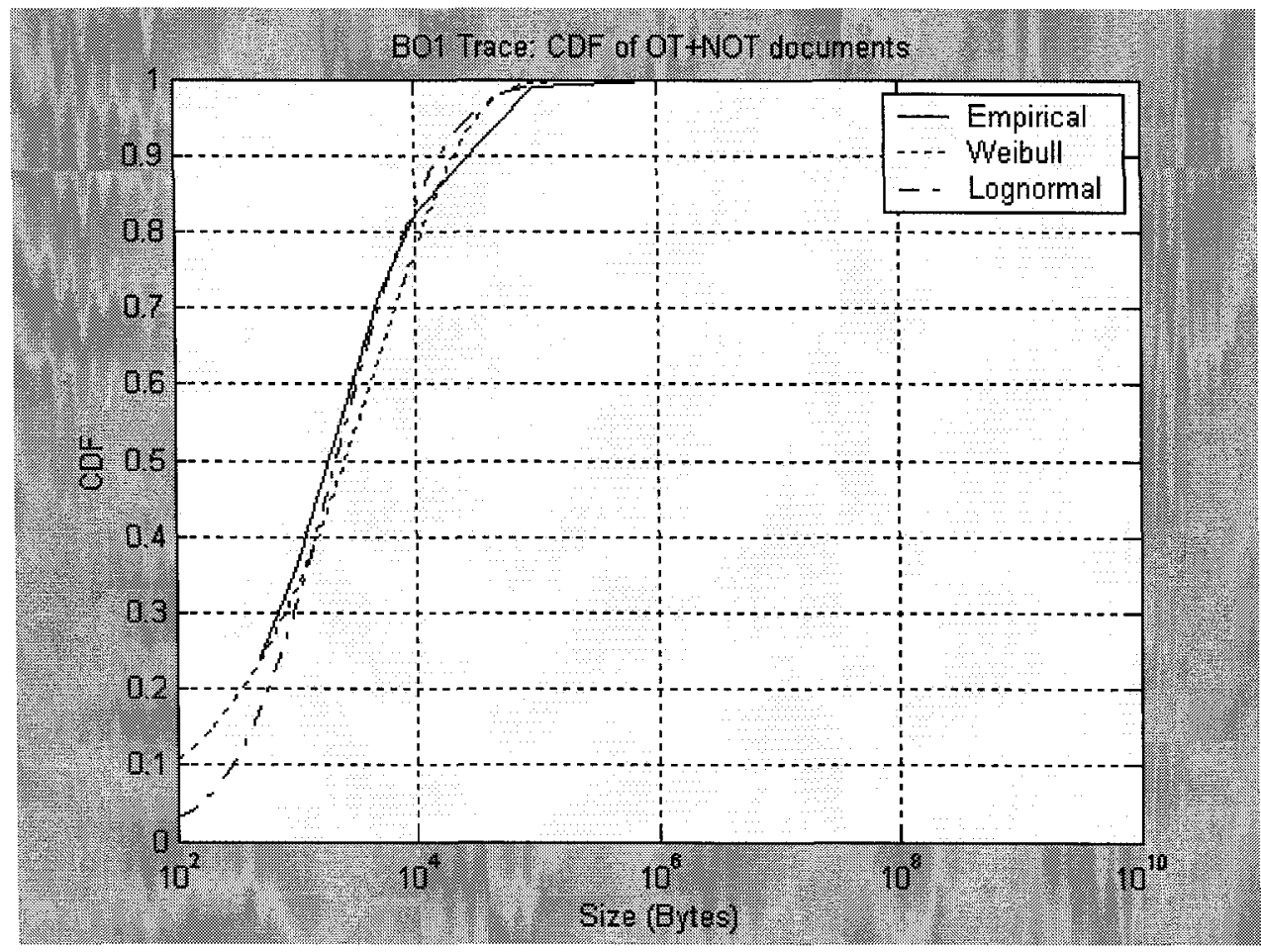

Figure 3.19: CDF of Total documents for BO1 trace

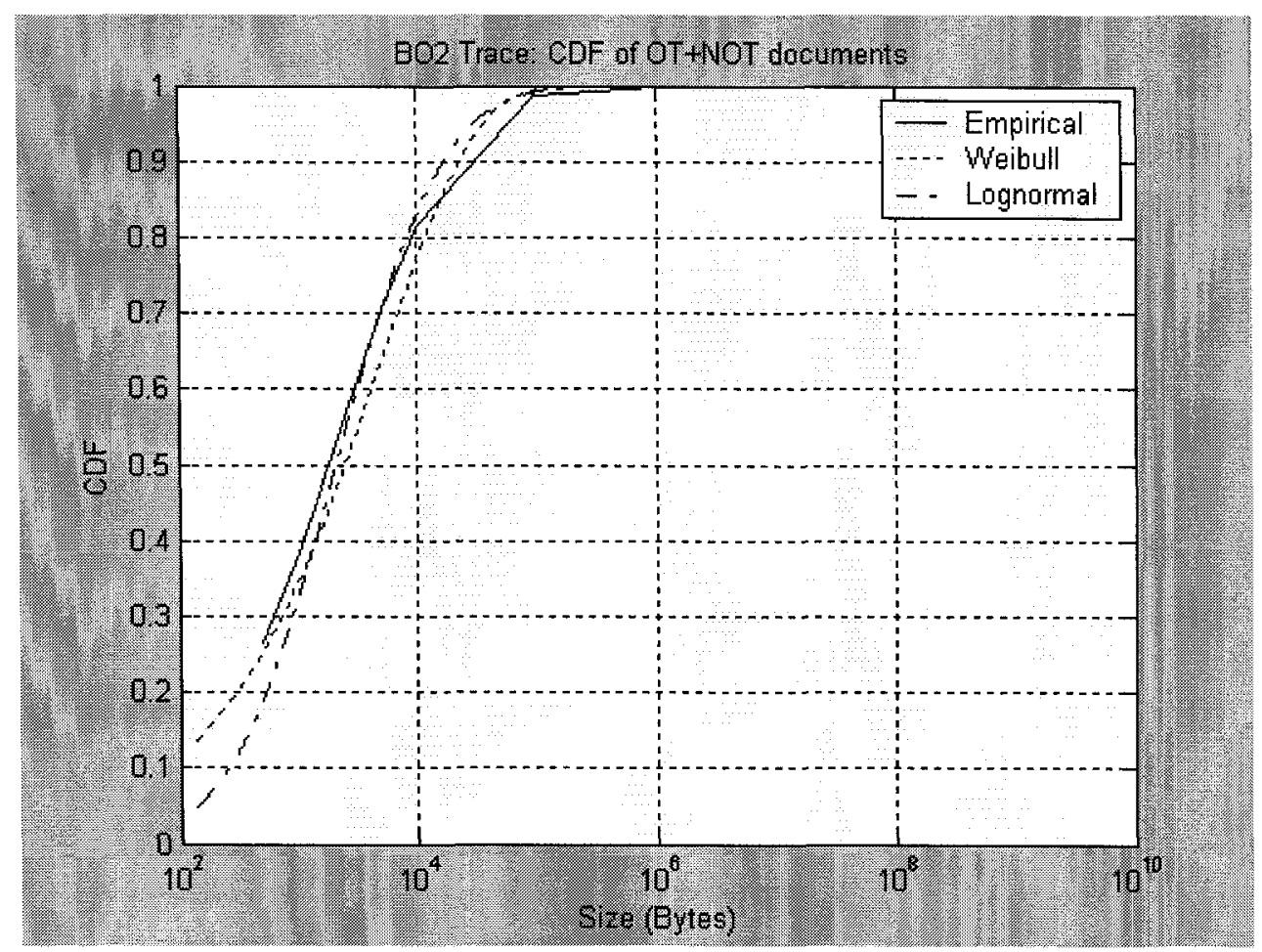

Figure 3.20: CDF of Total documents for $\mathrm{BO} 2$ trace 


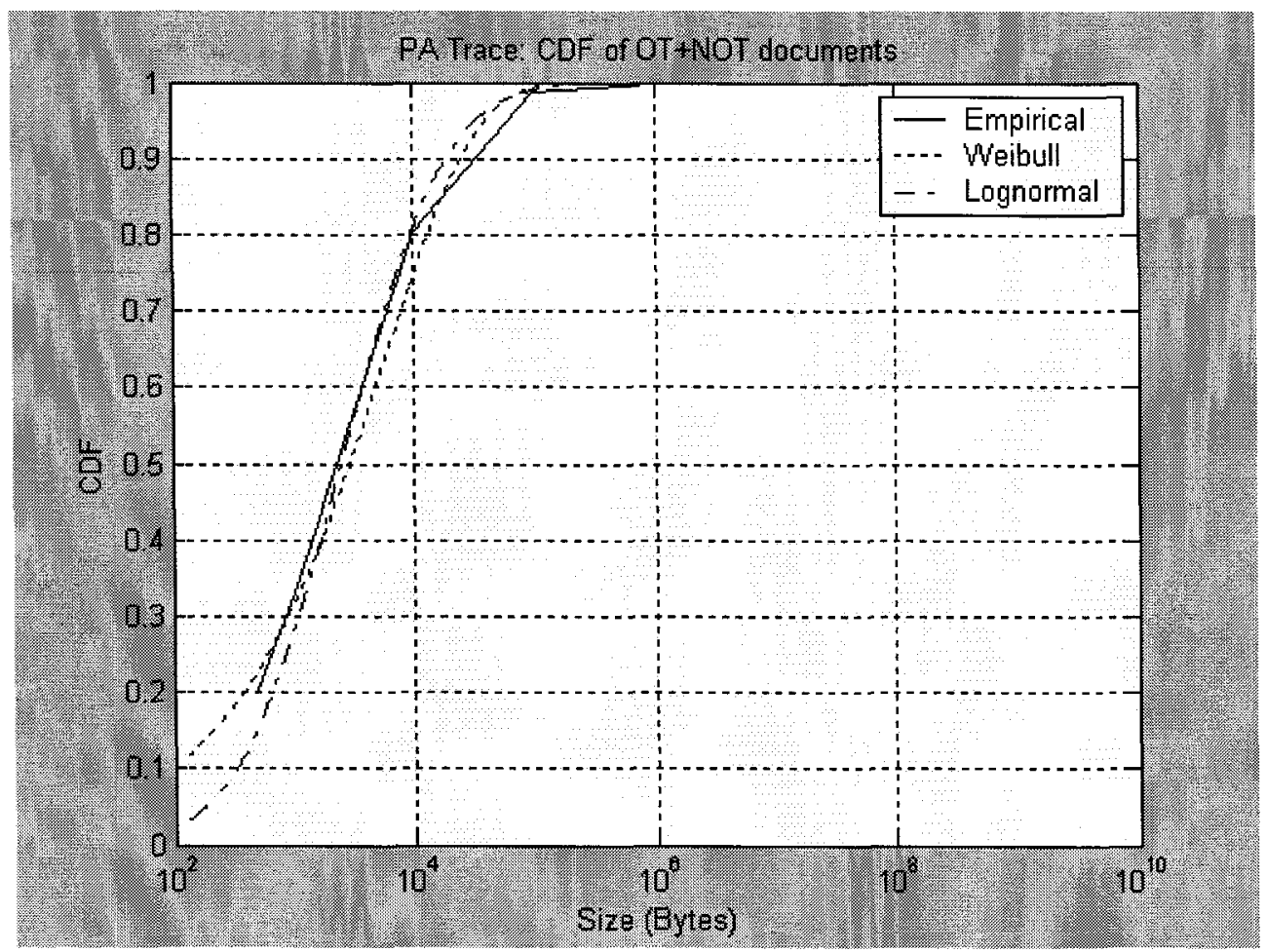

Figure 3.21: CDF of Total documents for PA trace

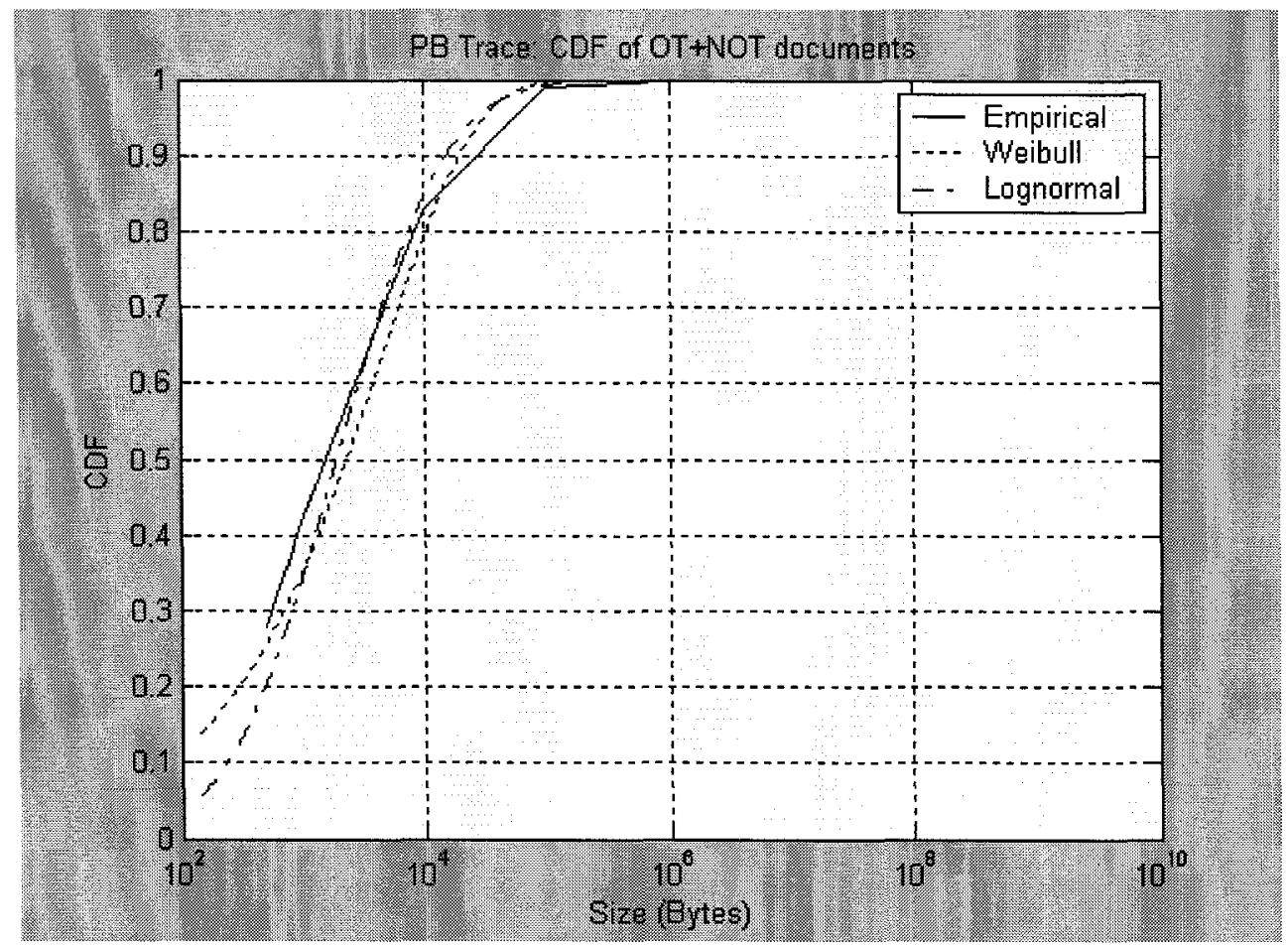

Figure 3.22: CDF of Total documents for PB trace 


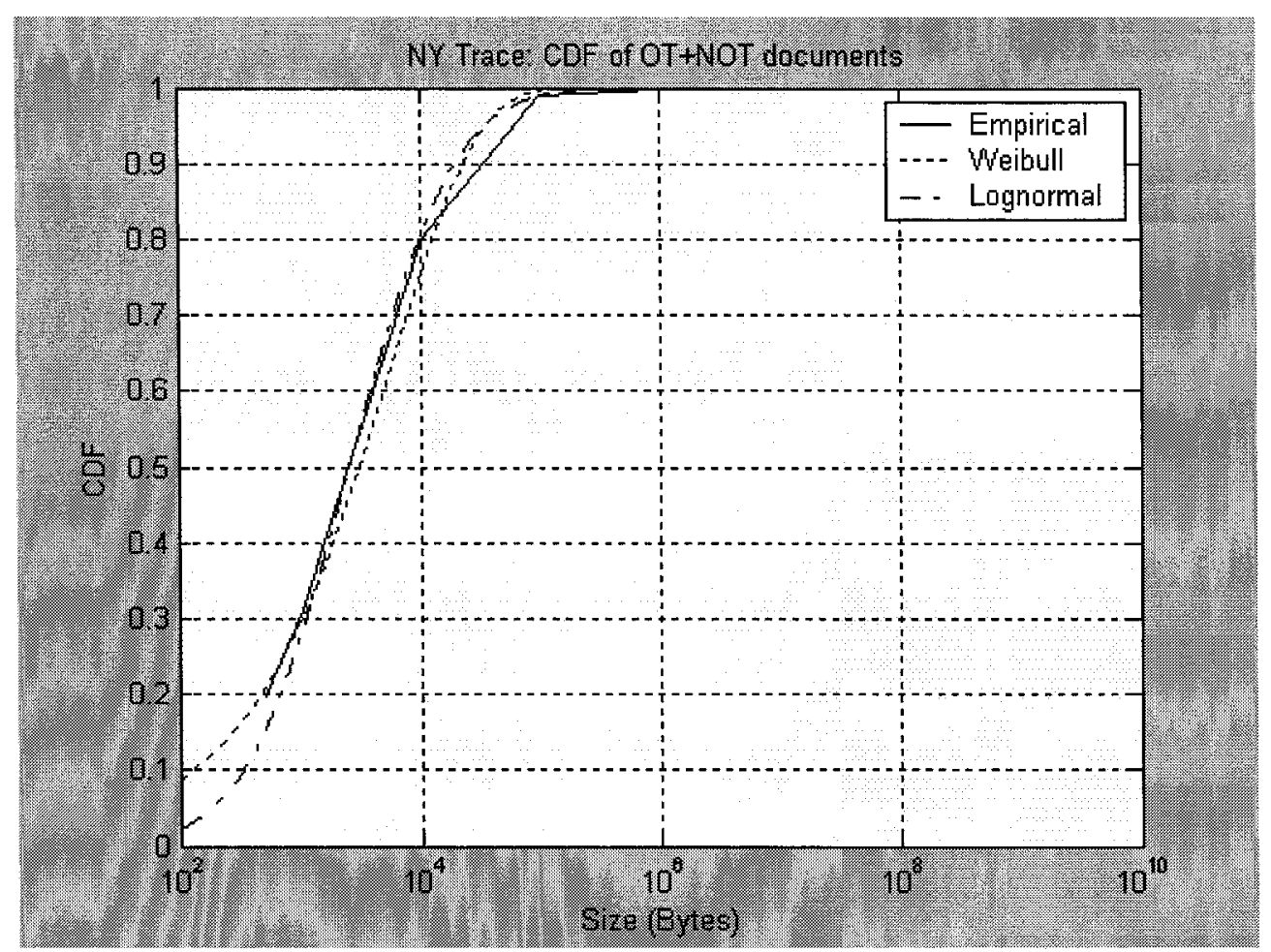

Figure 3.23: CDF of Total documents for NY trace

\subsubsection{Goodness of Fit}

The K-S goodness of fit test measures the largest vertical distance $d_{n}$ between the CDF of the fitted model and the empirical distribution of the data samples. The value of this distance is defined as:

$$
d_{n}=\text { Maximum of all } d_{n+} \text { and } d_{n-}(\geq 0) ; \text { for } i=1 \ldots n
$$

where $n$ is the number of samples in the data set and

$$
\begin{gathered}
d_{n+}=f_{n}\left(x_{i}\right)-f_{0}\left(x_{i}\right), \\
d_{n-}=f_{0}\left(x_{i}\right)-f_{n-1}\left(x_{i}\right), \\
f_{0}\left(x_{i}\right)=P_{0}\left(x \leq x_{i}\right)=\operatorname{CDF}\left(x_{i}\right), \text { and }
\end{gathered}
$$




$$
f_{n}\left(x_{i}\right)=\neq \text { of } x^{\prime} s \leq \frac{x_{i}}{n}=\frac{i}{n} ; i=1 \ldots n
$$

The value of $d_{n}$ shows the deviation between the model and data. Thus, a smaller value of $d_{n}$ indicates that the assumed CDF is more valid, while a larger value indicates that the underlying data distribution is not the best likely fit.

The calculated values of $d_{n}$ for the Weibull and lognormal models for each of the traces are shown for one-timer, non-one timer and total documents in Table 3.10. For both fitted models, $d_{n}$ values for total documents follow quite closely with one-timer behavior while they are much higher for non-one timers. For the total documents in the traces, $d_{n}$ values for the Weibull model range from 0.11 to 0.16 and for the lognormal model, they range from 0.04 to 0.09 . A comparison of these $d_{n}$ values indicates that the lognormal distribution is a better fit than the Weibull distribution for each of the seven traces considered in this analysis.

Thus, based on the graphs and the K-S statistics, we conclude that the lognormal provides the best visual fit for the one-timer, non-one timer and combined one-timer/non-one timer Web documents. 


\begin{tabular}{|c|c|c|c|c|c|c|}
\hline \multirow{2}{*}{ TRACE } & \multicolumn{2}{|c|}{ Total } & \multicolumn{2}{c|}{ OT } & \multicolumn{2}{c|}{ NOT } \\
\cline { 2 - 7 } & Weibull & lognormal & Weibull & lognormal & Weibull & lognormal \\
\hline UC1 & 0.154 & 0.076 & 0.136 & 0.059 & 0.263 & 0.201 \\
\hline UC2 & 0.165 & 0.088 & 0.147 & 0.069 & 0.270 & 0.205 \\
\hline BO1 & 0.150 & 0.072 & 0.140 & 0.062 & 0.239 & 0.175 \\
\hline BO2 & 0.163 & 0.079 & 0.152 & 0.068 & 0.263 & 0.203 \\
\hline PA & 0.147 & 0.058 & 0.136 & 0.048 & 0.256 & 0.206 \\
\hline PB & 0.159 & 0.094 & 0.147 & 0.085 & 0.228 & 0.166 \\
\hline NY & 0.113 & 0.042 & 0.102 & 0.034 & 0.255 & 0.245 \\
\hline
\end{tabular}

Table 3.10: Goodness of fit $d_{n}$ values for one-timer, non-one timer and total documents

\subsubsection{Heavy Tailed Property}

In order to examine if the CDF of documents for all the traces discussed in Section 3.3 and 3.3.1 has a heavy tailed distribution, a log-log complementary distribution (LLCD) plot was used. The LLCD distribution is the complementary of the CDF and amplifies the tail behavior. A cumulative distribution function $F(x)$ expresses the probability that a random variable $X$ is less than $x$. By definition, the complementary distribution is obtained by computing $1-F(x)$, which expresses the probability that a random variable $X$ exceeds $x$ [6]. An LLCD plot shows the value of $\bar{F}(x)$ versus $x$, using logarithmic scales on both axes. 


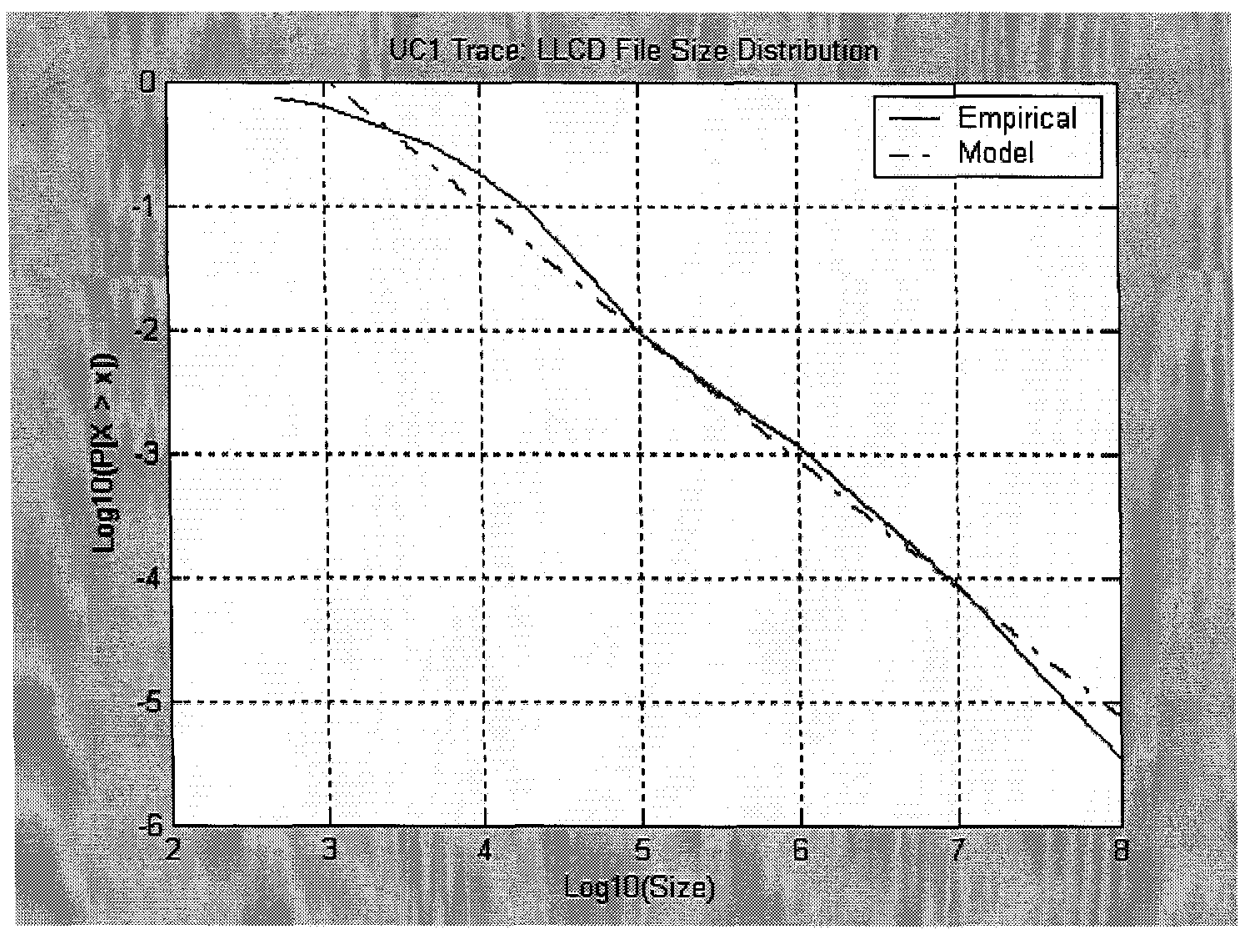

Figure 3.24: LLCD of documents size for UC1 trace

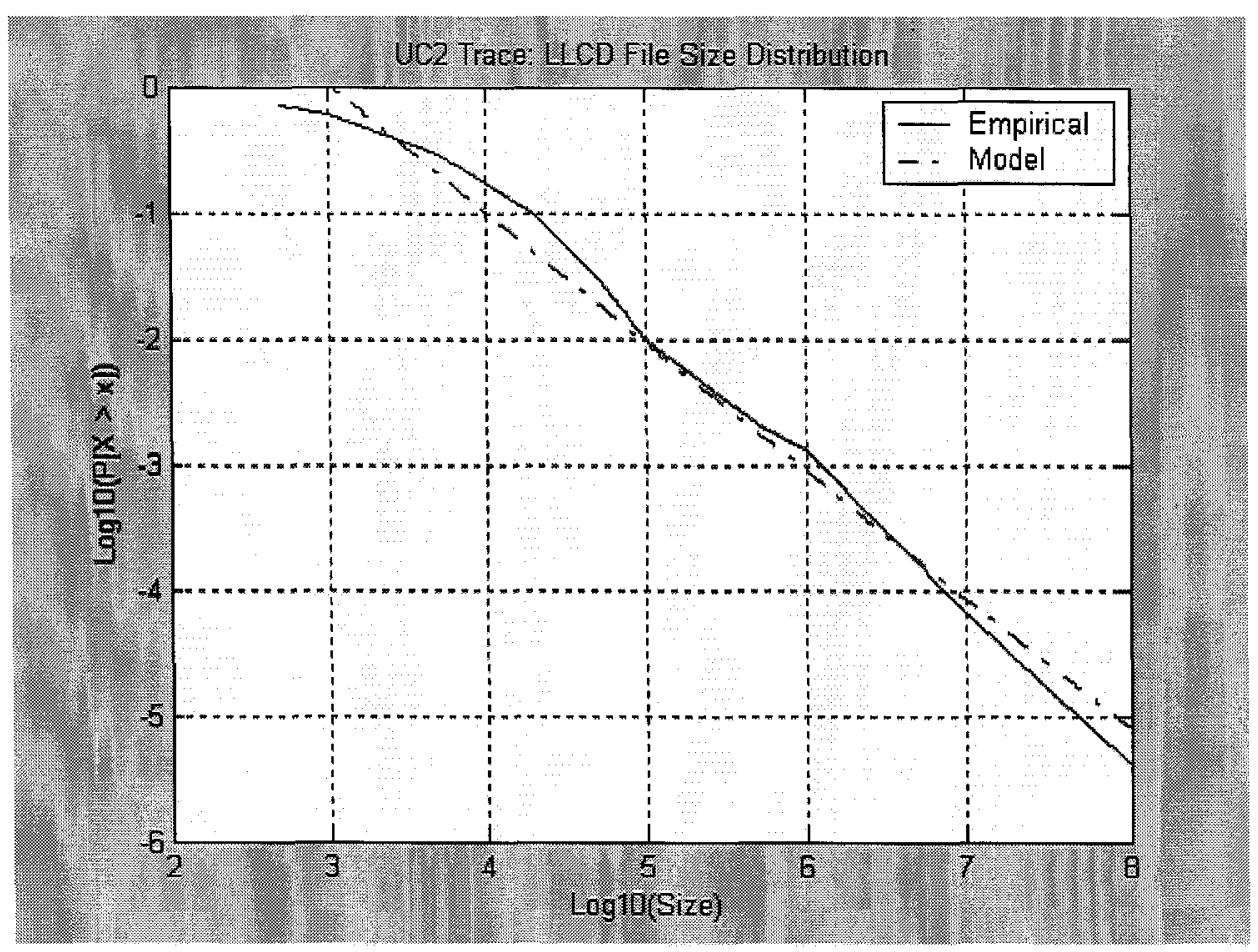

Figure 3.25: LLCD of documents size for UC2 trace 


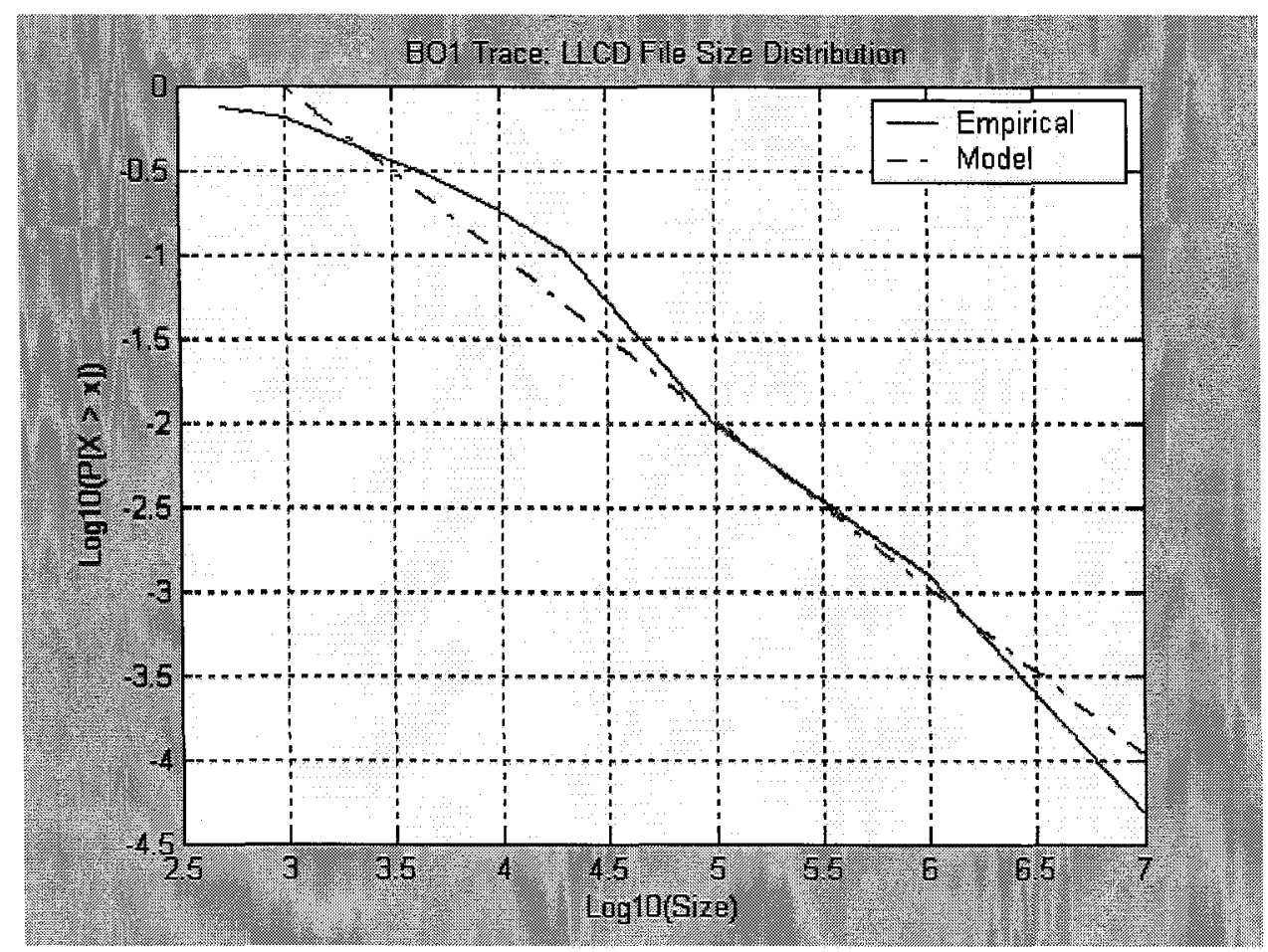

Figure 3.26: LLCD of documents size for BO1 trace

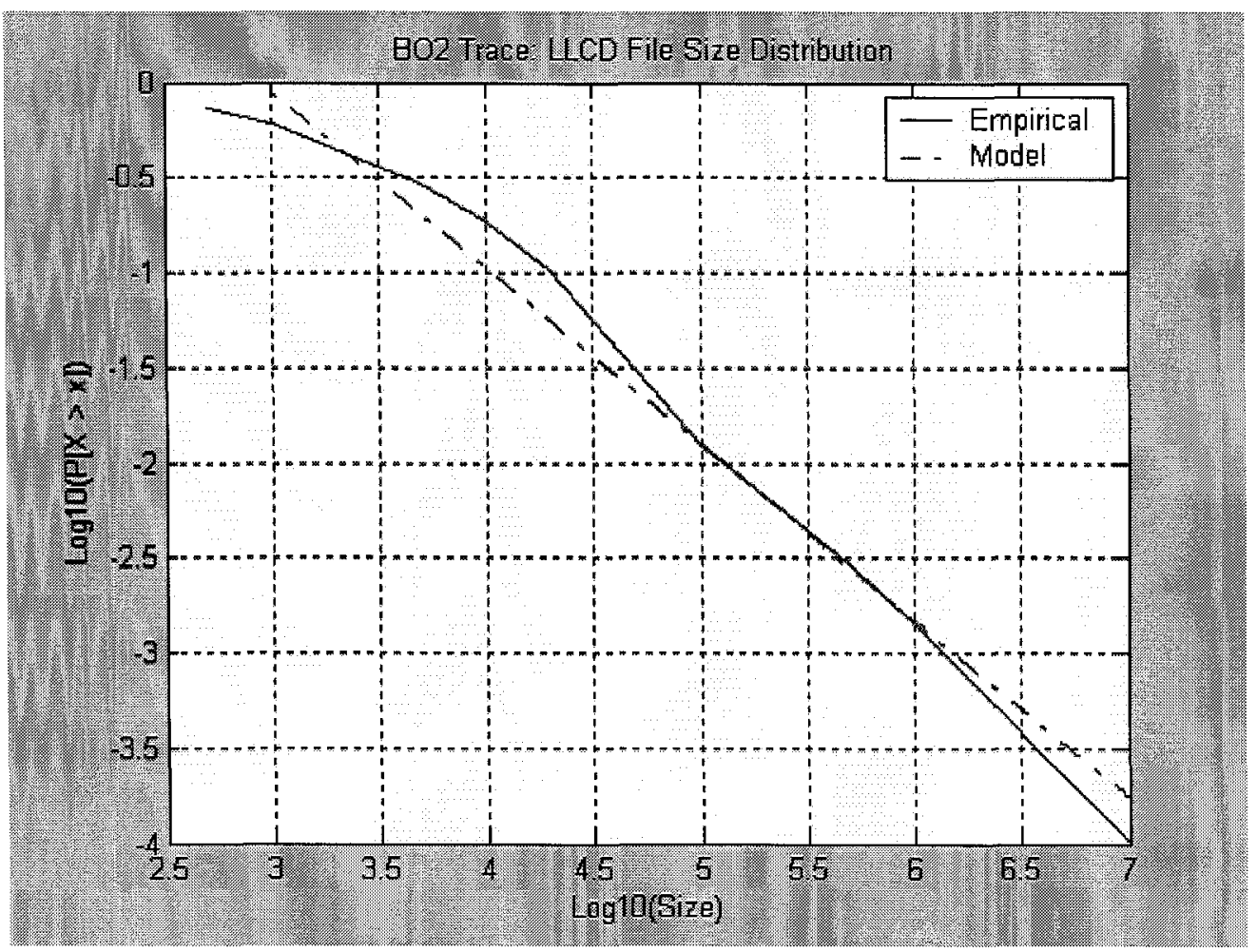

Figure 3.27: LLCD of documents size for $\mathrm{BO} 2$ trace 


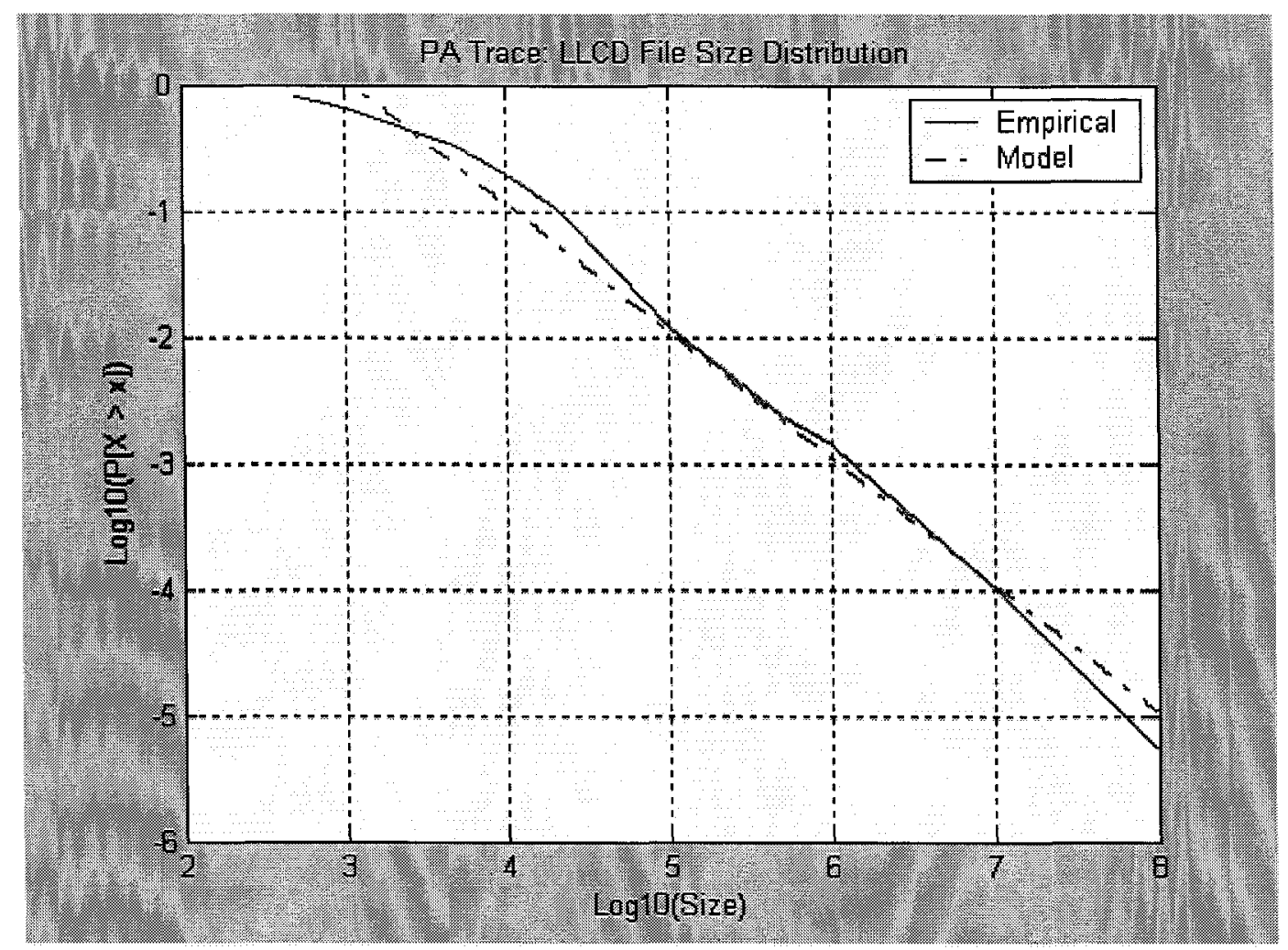

Figure 3.28: LLCD of documents size for PA trace

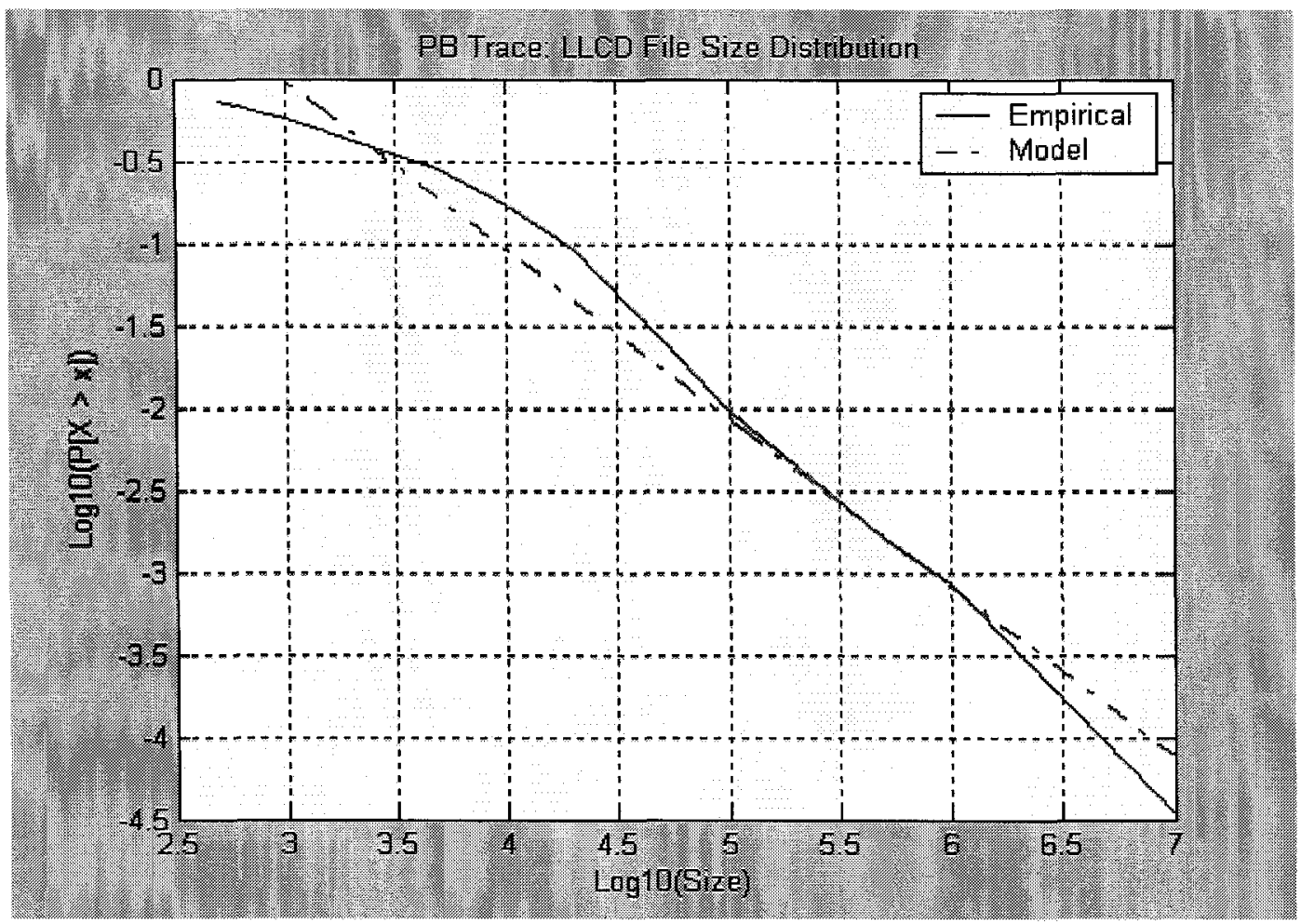

Figure 3.29: LLCD of documents size for PB trace 


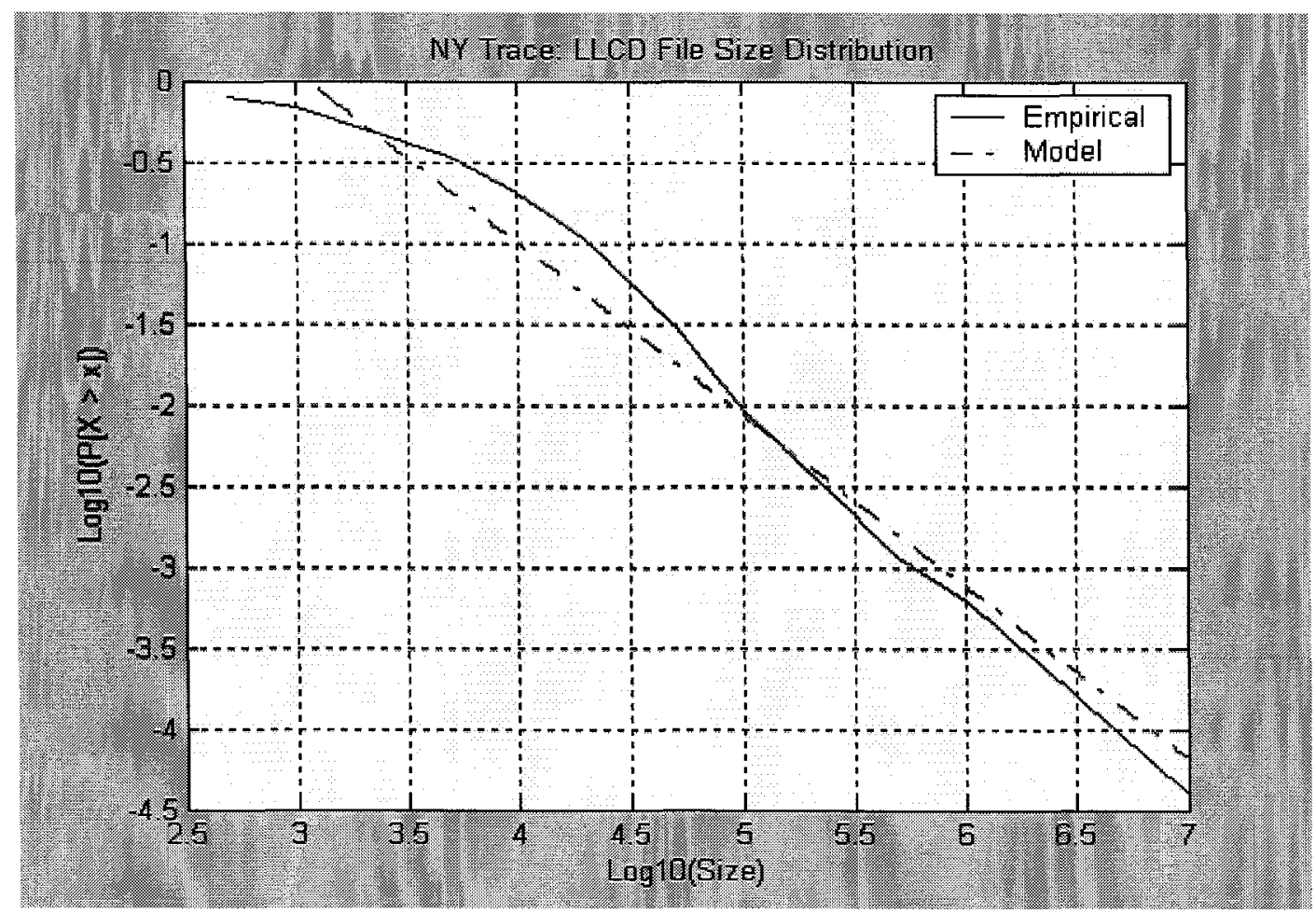

Figure 3.30: LLCD of documents size for NY trace

Figures 3.24 to 3.30 show the LLCD plot based only on the size of total documents for all the NLANR traces considered. On an LLCD plot, a heavy-tailed distribution typically manifests itself with straight-line behavior with slope $\alpha$. This is evident in Figures 3.24 to 3.30 for all the traces. A least squares linear regression fit is applied to the empirical models for each of the traces to estimate the $\alpha$ (i.e. slope) and $R^{2}$ values (i.e. coefficient of determination). A value of $\alpha$ between 0 and 2 indicates a heavy tailed distribution, and a value of $R^{2}$ that is closer to 1.0 indicates a stronger fit, which implies a linear behavior.

Table 3.11 shows $\alpha$ and $R^{2}$ values obtained for one-timer, non-one timer and total documents separately in all the traces considered. It can be seen from Table 3.11 that $\alpha$ 
value is in the range of 0.95 to 1.08 for one-timer documents, 0.77 to 0.92 for non-one timer documents and 0.93 to 1.05 for total documents. The $\alpha$ value for non-one timer documents is lower when compared to the one-timer documents, indicating a heavier tail for the non-one timers. This is due to the concentration of few non-one timer documents resulting in a large amount of data transferred as noted in the tail of their CDF. Thus it can be concluded that the heavy tailed distribution for total documents in all the traces is predominantly influenced by the one-timer documents.

\begin{tabular}{|c|c|c|c|c|c|c|c|}
\hline Item & UC1 & UC2 & $\mathrm{BO} 1$ & $\mathrm{BO} 2$ & $\overline{P A}$ & PB & $\overline{N Y}$ \\
\hline \multicolumn{8}{|c|}{ One Timer Documents } \\
\hline $\bar{\alpha}$ & 1.03 & 1.03 & 1.03 & 0.95 & 1.01 & 1.05 & 1.08 \\
\hline$R^{2}$ & 0.98 & 0.98 & 0.97 & 0.97 & 0.98 & 0.97 & 0.96 \\
\hline \multicolumn{8}{|c|}{ Non-one Timer Documents } \\
\hline$\alpha$ & 0.90 & 0.83 & 0.80 & 0.78 & 0.77 & 0.92 & 0.85 \\
\hline $\mathrm{R}^{2}$ & 0.91 & 0.95 & 0.96 & 0.97 & 0.97 & 0.95 & 0.96 \\
\hline \multicolumn{8}{|c|}{ Total Documents } \\
\hline$\alpha$ & 1.02 & 1.02 & 0.99 & 0.93 & 0.99 & 1.02 & 1.05 \\
\hline $\mathrm{R}^{2}$ & 0.98 & 0.98 & 0.97 & 0.98 & 0.98 & 0.97 & 0.97 \\
\hline
\end{tabular}

Table 3.11: Estimate of $\alpha$ for size of One-Timer, Non-one Timer and Total documents

\subsection{Popularity Characterization}

As discussed in Section 2.2, the popularity of documents in the traces can be characterized by a distribution similar to the Zipf (i.e. Zipf-like). A Zipf-like distribution expresses a power law relationship between the Popularity $(P)$ of a document and its rank $r$ as follows: $P=\frac{c}{r^{\beta}}$ where $c$ is a constant. The shape of the distribution is linear with the slope equal to $-\beta$ on a $\log -\log$ plot of popularity $v s$ rank [14]. 
If $\beta$ is exactly equal to unity, it is referred to as Zipf's law and if it is close to unity, it is referred to as a Zipf-like distribution [14]. The value of $\beta$ is important because it affects the popularity of the curve. The popularity curve shows the concentration of references to the popular documents that affects the cache performance. The value of $\beta$ depends on the location of a cache in a hierarchy. It has been observed that the higher-level proxies have values of $\beta$ which are smaller when compared to the lower level proxies in a cache hierarchy.

In order to examine whether the popularity of the documents requested in each trace follows a Zipf-like distribution, the number of references to the documents versus rank was plotted for each trace. Figures 3.24 to 3.30 show the log-transformed plot of the number of references to each document as a function of its rank in popularity. In these figures, the plots of the measured data in dotted line are referred to as empirical plots. The model represented by solid line in these Figures refers to the least-squares regression fit obtained for the empirical data. 


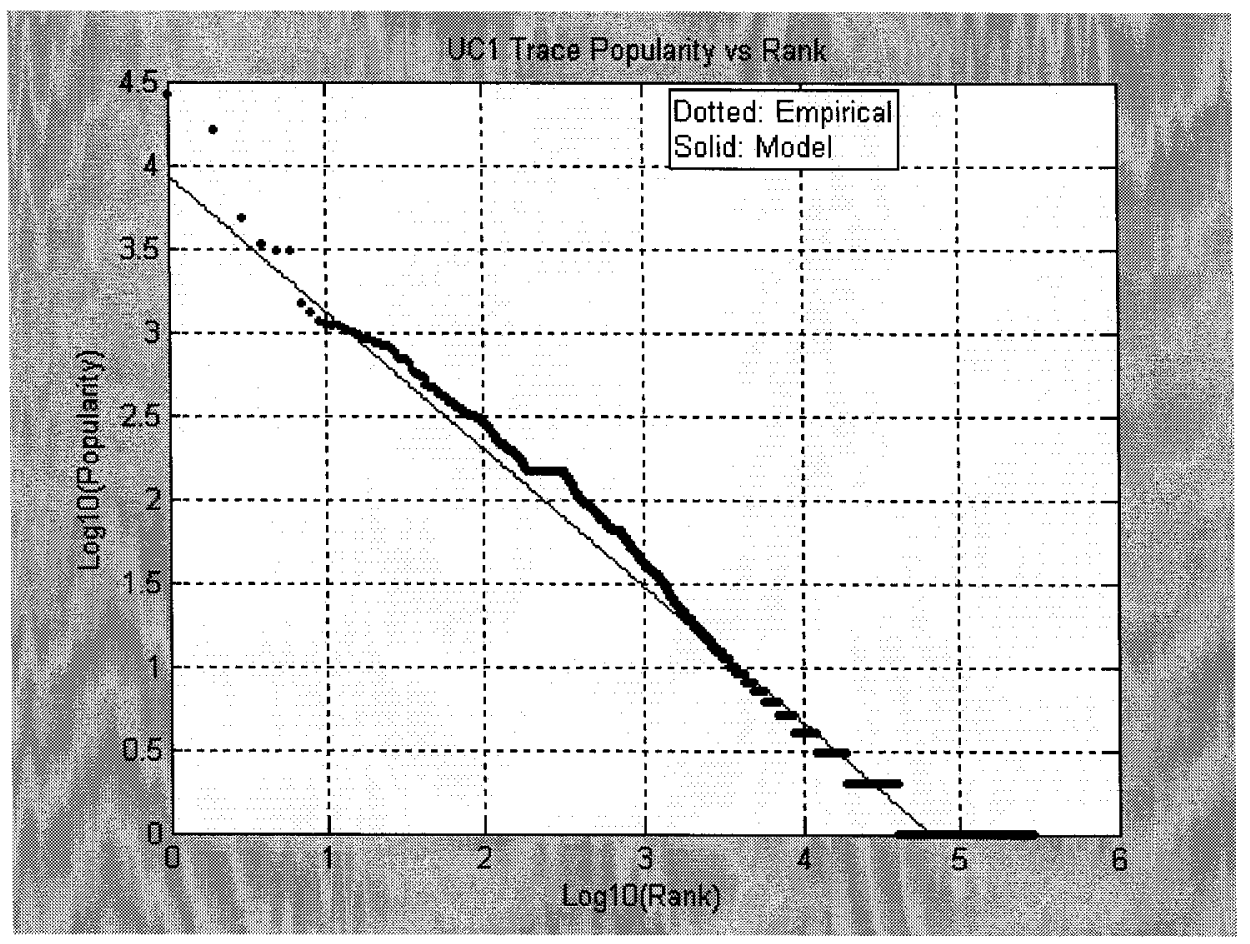

Figure 3.31: Popularity vs rank for UC1 trace

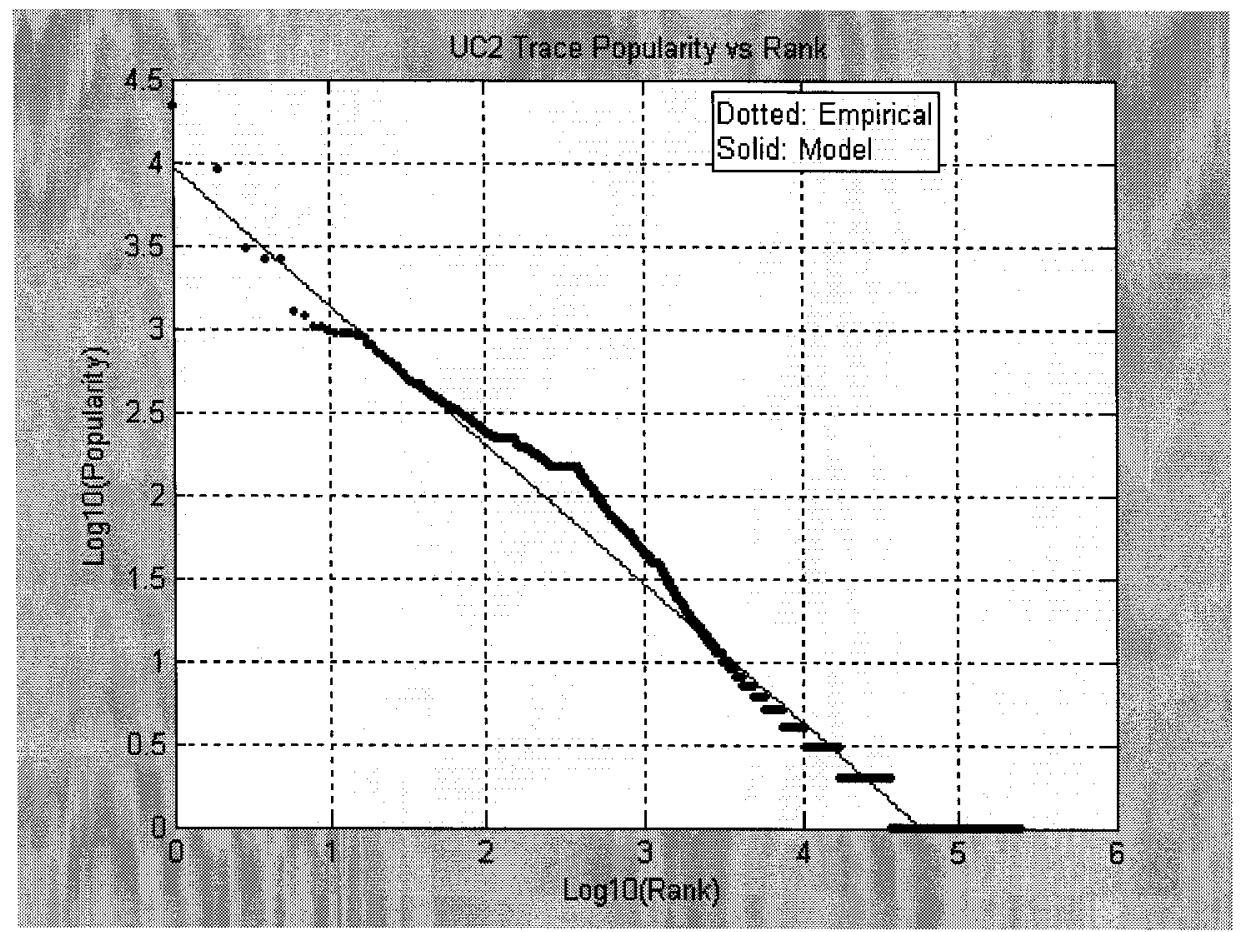

Figure 3.32: Popularity vs rank for UC2 trace 


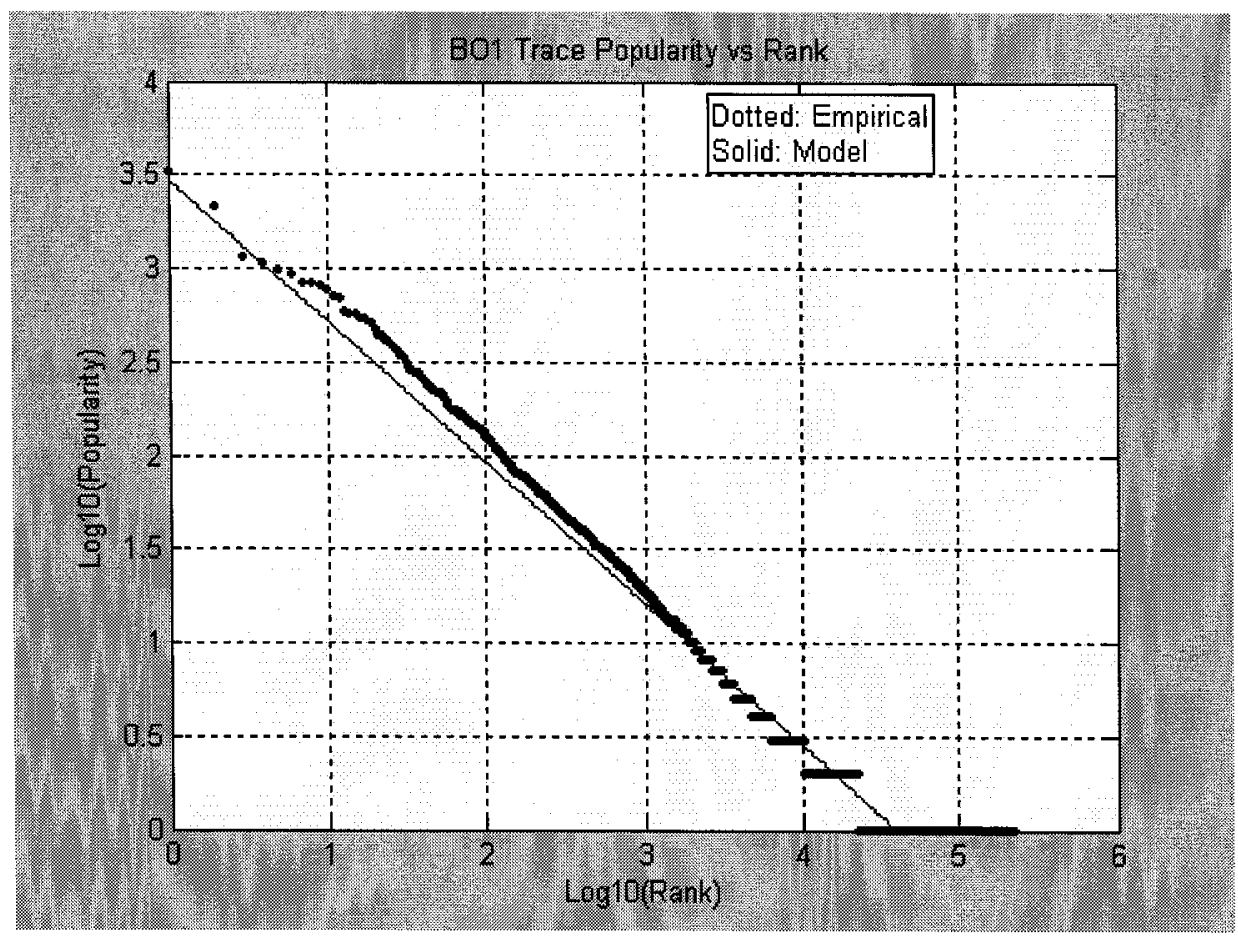

Figure 3.33: Popularity vs rank for BO1 trace

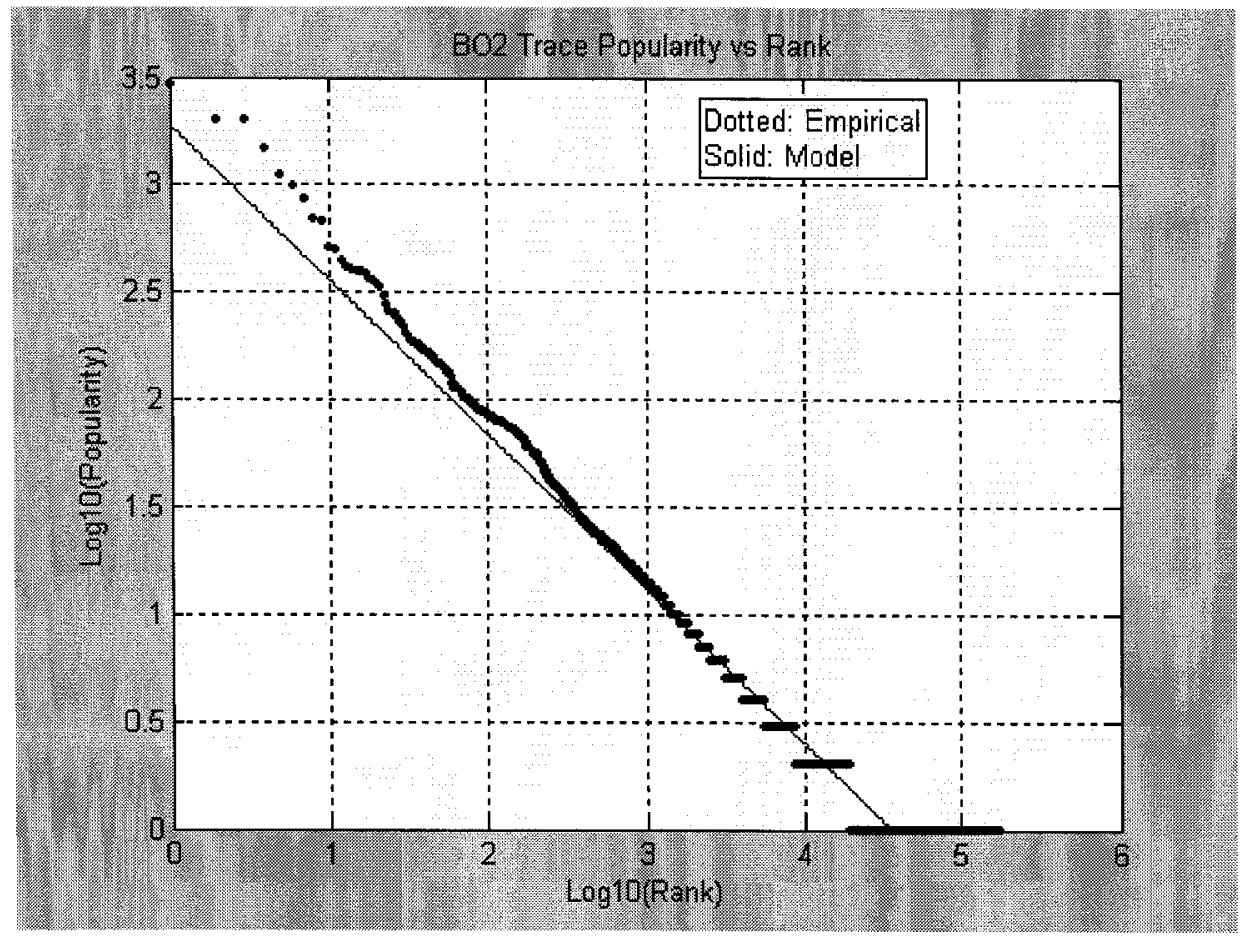

Figure 3.34: Popularity vs rank for $\mathrm{BO} 2$ trace 


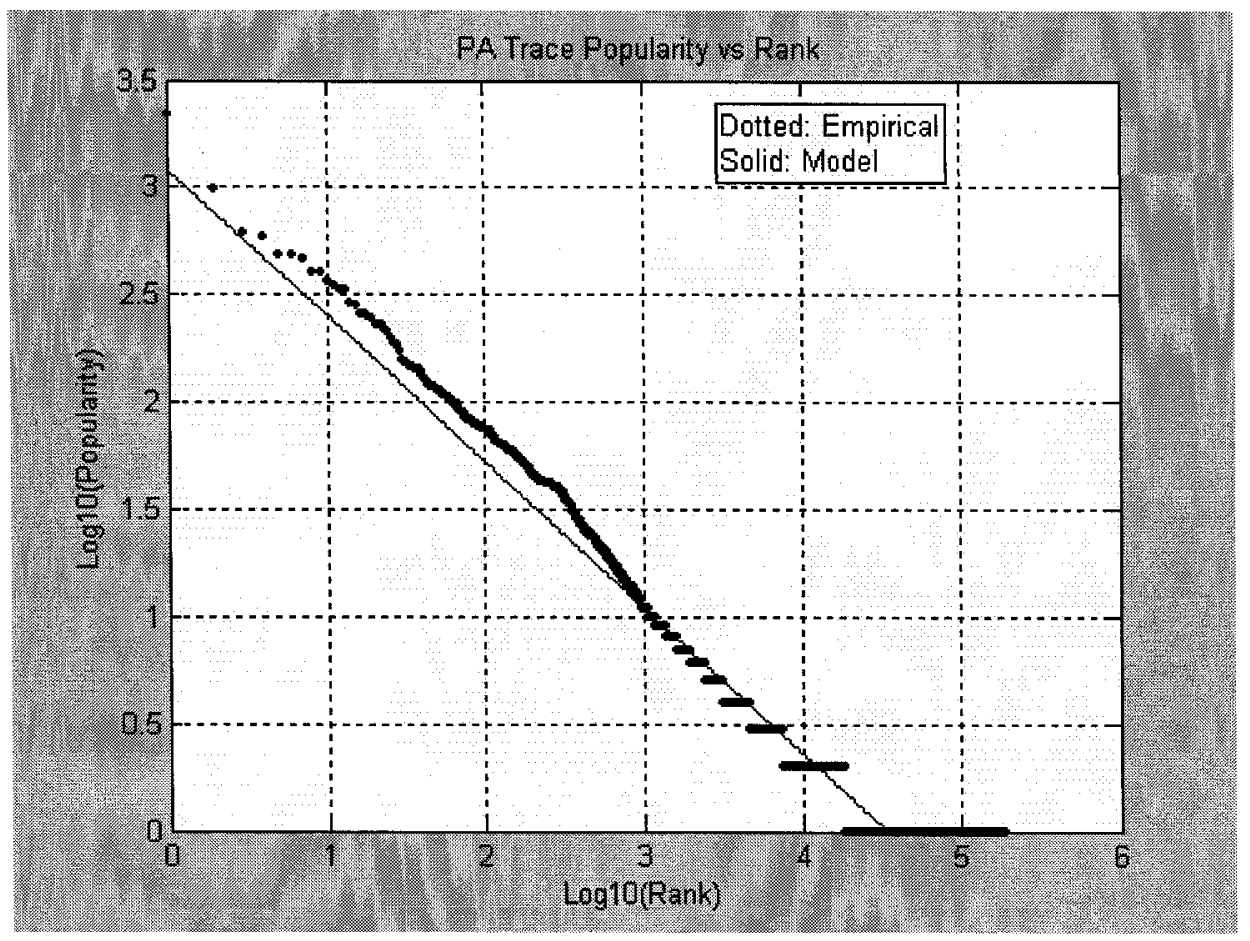

Figure 3.35: Popularity vs rank for PA trace

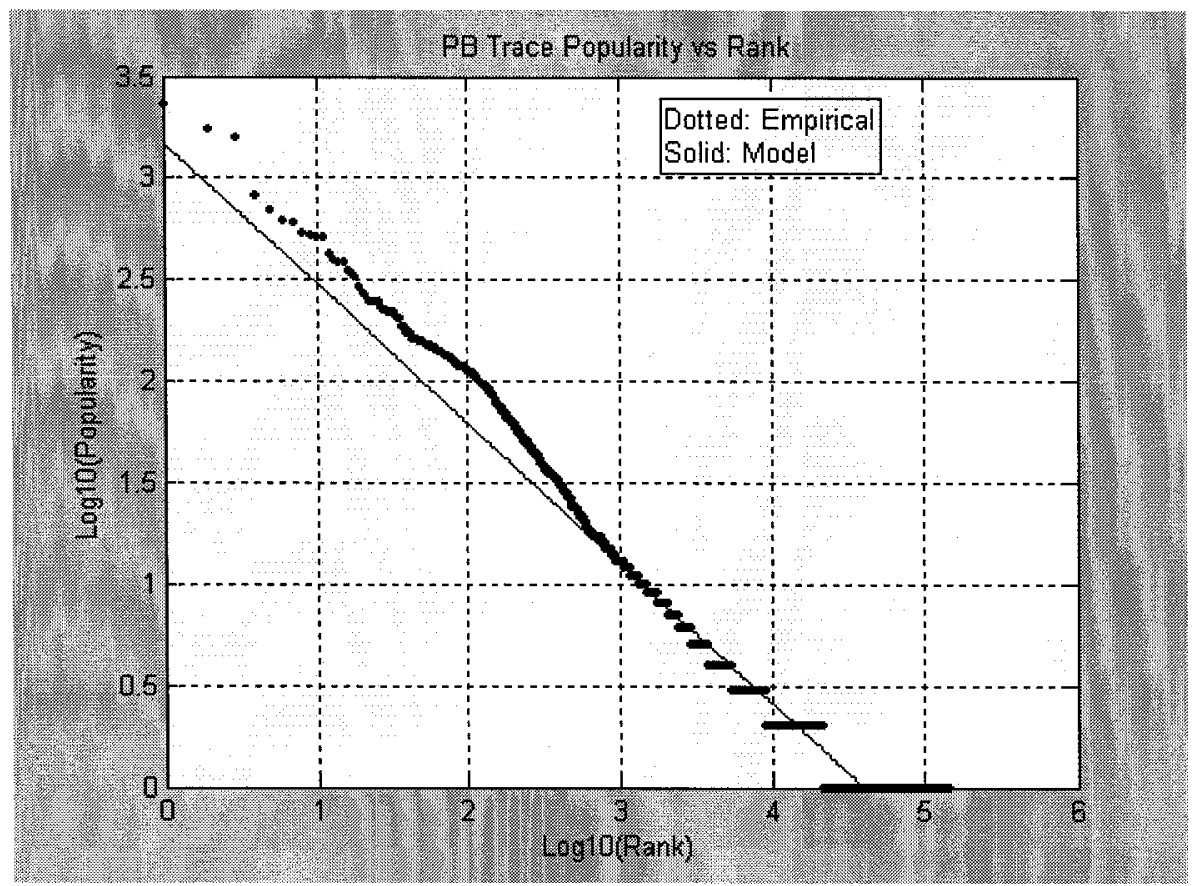

Figure 3.36: Popularity vs rank for PB trace 


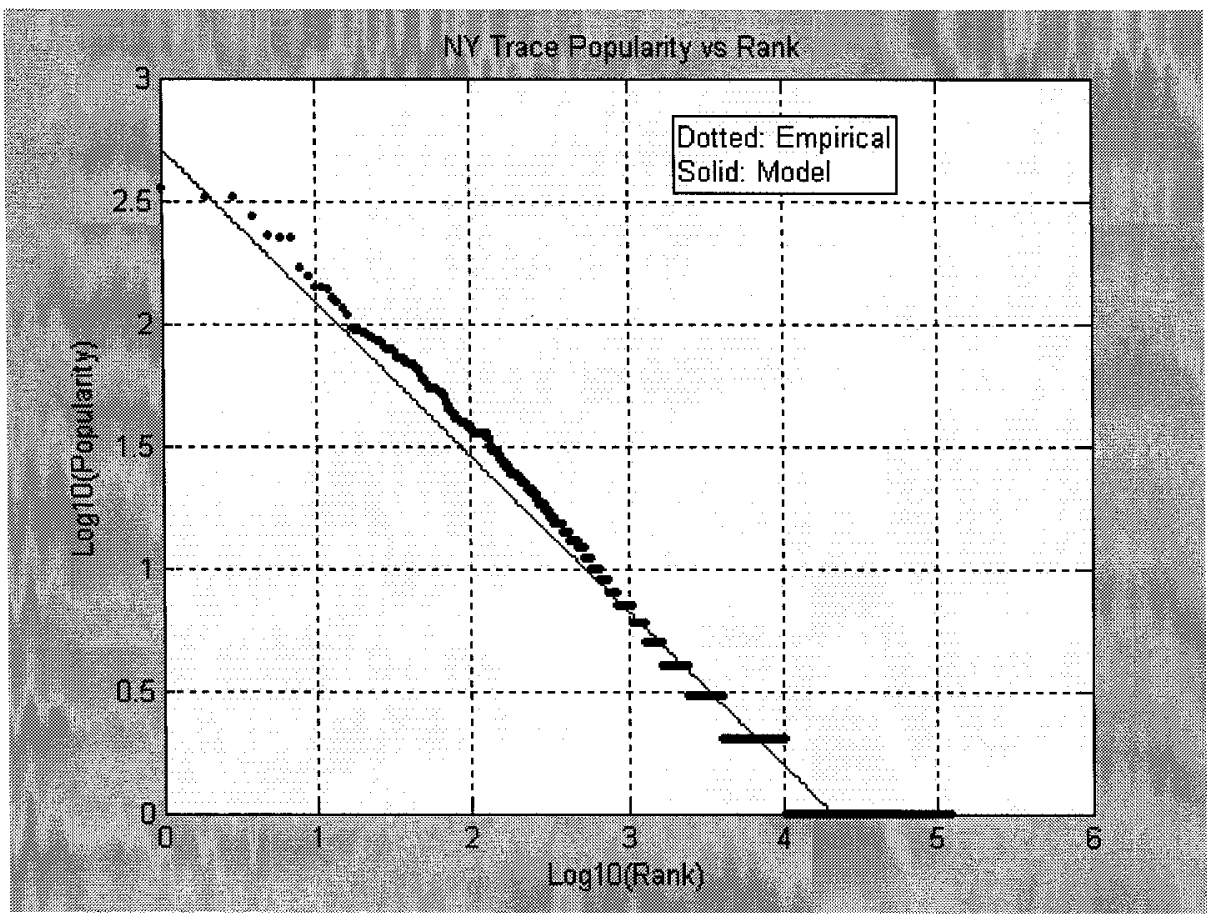

Figure 3.37: Popularity vs rank for NY trace

The calculated values for $\beta$ and the goodness of fit $R^{2}$ values for the seven traces are shown in Table 3.12. The value of $\beta$ is in the range of 0.63 to 0.84 for all the traces. It is evident from Figures 3.30-3.36 that the document referencing behavior does not exactly follow Zipf's law, as shown by the lower $\beta$ values. It can also be observed that the leastsquares fit and empirical distribution do not agree very strongly due to the presence of many documents of low priority and many one-timers. This confirms the hypothesis that the popularity patterns for these traces are characterized by Zipf-like distributions, although they do not precisely match a true Zipf distribution. 


\begin{tabular}{|c|c|c|c|c|c|c|c|}
\hline Item & UC1 & UC2 & BO1 & BO2 & PA & PB & NY \\
\hline$\beta$ & 0.82 & 0.84 & 0.76 & 0.72 & 0.68 & 0.69 & 0.63 \\
\hline $\mathrm{R}^{2}$ & 0.96 & 0.94 & 0.96 & 0.96 & 0.95 & 0.95 & 0.94 \\
\hline
\end{tabular}

Table 3.12: Popularity characteristics for all NLANR traces

It should be noted from Table 3.12 that higher values of $\beta$ for $\mathrm{UC} 1$ and $\mathrm{UC} 2$ should potentially result in better cache performance compared to the other traces. Similarly, we expect that $\mathrm{BO} 1$ and $\mathrm{BO} 2$ traces with higher values of $\beta$ compared to $\mathrm{PA}, \mathrm{PB}$ and $\mathrm{NY}$ traces will result in better cache performance.

\subsection{Summary}

This Chapter has discussed the Web workload characteristics by separating the one-timer and non-one timer documents in terms of their transfer size distribution and popularity. Trace driven analyses were used to study the statistical properties of one-timer and nonone timer documents independently, in detail, with the help of mathematical models and K-S goodness of fit distance tests. The Weibull and lognormal distributions were used to fit the empirical CDF data for one-timer, non-one timers and combined documents separately. Estimation of model parameters were obtained using 95\% confidence level bounds and it was shown that the lognormal distribution provided a superior fit to the empirical CDF obtained for each of the seven traces. 
Popularity characteristics for each of the traces were studied separately using empirical and mathematical models. It was shown that the documents popularity followed a Zipflike distribution due to the presence of many one-timer and low priority documents.

Thus by studying the properties of one-timer, non-one timer documents and comparing them to the combined documents in changing Web traffic patterns, we conclude that Web document characteristics are predominantly influenced by their one-timer distributions and exhibit self-similarity in their workloads. These workload characteristics for the recent traces considered are useful in designing cache replacement policies that take into account document size as an admission constraint parameter.

A new cache replacement policy using document size as an adaptive filter is discussed in Chapter 4. Using the seven NLANR traces, the cache performance is characterized in terms of their document hit ratios and their byte hit ratios. 


\section{Adaptive Admission Control Policy}

\subsection{Introduction}

Proxy cache performance can be effectively characterized by replacement policies that yield a high document hit ratio. Due to the storage space restrictions in the servers, the cache replacement policies have to effectively remove a number of cached documents to allow for a recently referenced one. As the use of the Internet grows exponentially and user traffic patterns change dynamically with time, efficient design of cache replacement policies are essential to improve the user response time by reducing the latency. In this Chapter we propose a new cache replacement policy, which uses the document size as an adaptive filter and the document hit ratio as a feedback signal.

The remainder of this Chapter is organized as follows. Section 4.2 provides the definition and a brief overview of the key replacement policies and the motivation behind the design of a new replacement policy. Section 4.3 describes the algorithm based on the new adaptive feedback admission control policy proposed in this study. In Section 4.4, cache

performance metrics are analyzed using the proposed policy. This Section also provides a comparison of the cache performance metrics using standard LRU and LRU-Threshold policies. Simulation results based on the analysis of NLANR traces are also discussed in this section. A summary of the Chapter is presented in Section 4.5. 


\subsection{Replacement Policies}

As we have already seen, the increasing demand for the WWW and converged multimedia services has made document caching a necessity to improve user response times and reduce Internet traffic. Caching provides an efficient means of reducing latency by bringing documents closer to clients and could be deployed at various points in the Internet: within the client's browser, at or near the server to reduce the server load, or at a proxy server.

The objectives of proxy caching are two fold: first, to reduce the access latency for a document; second, to reduce the amount of traffic that is sent across the network (from servers to clients), which also reduces the user's perceived latency. A proxy cache may have limited storage in which it stores the most popular documents. Whenever the cache is full and the proxy needs to cache a new document, it has to decide which document to evict from the cache to accommodate the new document. The policy used for this eviction decision is referred to as the replacement policy.

Several WWW replacement policies have been proposed in recent studies including the traditional replacement policies such as the previously explained LRU and LFU [19], Key-based replacement policies such as LRU-Threshold, LRU-MIN [32] and Cost-based replacement policies such as GD Size and SLRU [58]. A brief overview of these policies has already been discussed in Section 2.3 of Chapter 2. The SLRU and LRU-Threshold replacement policies discussed in Section 2.4 of Chapter 2 used admission constraints to decide whether or not it is worthwhile caching a document in the first place. 
The SLRU policy uses the past history of few document accesses as an admission constraint to stop non popular documents. A drawback of this policy is that it considers only a limited set of documents based on a predetermined auxiliary cache size which is a small fraction of the total cache size.

The LRU-Threshold policy caches all documents that are less than a specified threshold size. In this policy, a fixed threshold is used as an admission constraint, and the standard LRU is used as the replacement policy. The LRU-Threshold policy is static in nature in terms of using its admission constraint.

\subsubsection{Effect of Cache Size}

The cache size indicates the amount of space available for storing Web documents. The number of distinct documents represents an upper bound on the number of documents that could be cached. The total size (bytes transferred) of these unique documents indicates the maximum useful (Infinite) cache size. When an infinite cache size is considered for any given access server log, the maximum document hit ratio can be achieved for that specific server $\log$ as there is no replacement. However, as traffic patterns change dynamically with the increasing usage of the Web and bandwidth demand grows, it is unrealistic to assume an infinite cache size due to the several physical, economical and performance constraints.

In a previous work reported in the literature [55], it was shown (using trace driven simulations) that $10 \%$ of the infinite cache size can achieve $80 \%$ to $90 \%$ of the maximum 
achievable hit ratio. With increasing cache sizes, the hit ratios tend to reach a steady state without being affected by any replacement policies.

None of the cache replacement policies discussed in the literature so far has dealt with an adaptive input admission constraints in order to optimize the cache performance. In this Thesis, we present a new approach to enhancing proxy caching, which takes into account the document size as an input tuning parameter, and the document hit ratio as a feedback mechanism so as to obtain a balance in the document hit ratio and the byte hit ratio.

\subsubsection{Performance Metrics}

Replacement policies rely on key metrics to achieve their goals. These policies attempt to optimize various performance metrics, including the document hit ratio, the byte hit ratio, the average download time, and the delay saving ratio [73]. In this study the performance of proxy cache is evaluated using two key metrics: the document hit ratio and the byte hit ratio.

\section{Hit ratio:}

The document hit ratio is calculated as the ratio of the number of cache hits to the total number of cache requests and is expressed as a percentage. Formally, this hit ratio can be expressed as follows:

$$
h_{r}=\frac{\sum_{i \in R} h_{i}}{\sum_{i \in R} f_{i}}
$$




\section{Byte hit ratio:}

The document byte hit ratio is the proportion of the total number of bytes that the proxy cache was able to serve directly by providing the information from its cache. It is the ratio of the sum of sizes of the requested documents found in the cache and sum of the sizes of all the requested documents. Mathematically, the byte hit ratio can be expressed as follows:

$$
b h_{r}=\frac{\sum_{i \in R} s_{i} \cdot h_{i}}{\sum_{i \in R} s_{i} \cdot f_{i}}
$$

In (4.1) and (4.2), $s_{i}$ refers to the size of document $i, f_{i}$ refers to the total number of requests for document $i, h_{i}$ is the total number of hits for document $i$, and $R$ is the set of all accessed documents. The document hit ratio is the most important performance metric, since a higher value for this index implies that more client requests will see a low latency. Thus the hit ratio is used as a figure of merit in evaluating the cache performance in our study.

\subsection{Adaptive Feedback Policy}

In this Section we introduce an adaptive feedback admission control policy to improve the performance of proxy caches using NLANR traces. The cache performance is evaluated using the performance metrics discussed above in Section 4.2.2. This policy considers the document size threshold as an adaptive admission constraint in conjunction 
with the LRU replacement policy to obtain a balance between the document hit ratio and the byte hit ratio.

The adaptive feedback policy algorithm that we implemented in our simulation model is given in Figure 4.1. This algorithm uses a linked list object for managing the cache. The most recently used document is in the front and the least recently used document is in the end of the list. In this algorithm, we start with an initial threshold which is considered to be the same as the threshold value for LRU-Threshold policy.

In Figure 4.1, we use $\mathrm{q}$ for referring to a requested document. For each request to $\mathrm{q}$, if it exists in the cache, we compare the size of the document in the cache with the requested document q. If the two sizes are same, we move the document to the front of the list. If the two sizes are different, it means that the requested document has already been updated. We thus update the document in cache and move the document to the front of the list.

If the requested document $\mathrm{q}$ is not present in the cache, we check if there is enough room in the cache to store it. If there is room in the cache, we add the document $\mathrm{q}$ to the front of the list. However, if there isn't enough room in the cache, we compare the size of the requested document $\mathrm{q}$ with the threshold size. If the size of the requested document is less than the threshold size, we evict the least recently used documents from the cache, and then add the requested document $\mathrm{q}$ to the front of the list. Otherwise, we ignore the document and proceed to the next request. 
To analyze the cache performance with an adaptive input admission constraint, we first divide the NLANR trace into intervals of certain fixed number of references. For our experiments, we have partitioned each trace into $\mathrm{N}$ intervals of 2500 document references. By doing so, we intuitively hope that it is possible to build a feedback learning system that helps us in understanding and analyzing access patterns with respect to time.

For the first interval of 2500 documents requested, an initial size threshold is fixed based on the chosen cache size for simulation. With this size threshold as an input constraint, the LRU replacement policy discussed in Section 4.2 is used to calculate the document hit ratio for that first interval. For the second interval, the above process of admission and replacement is repeated to obtain the document hit ratio. The hit ratio obtained in the second interval is then compared to the hit ratio during the first interval.

The performance delta, in hit ratios between the two intervals is taken as a feedback constraint in varying the size threshold for the subsequent $\mathrm{N}-2$ intervals. If the performance gets better (larger by 1\%), the size threshold is increased. Otherwise, the threshold is decreased. This process is repeated for each of the intervals in the entire trace duration, and finally, a cumulative document hit ratio and byte hit ratio based on $\mathrm{N}$ intervals is obtained for the trace. 


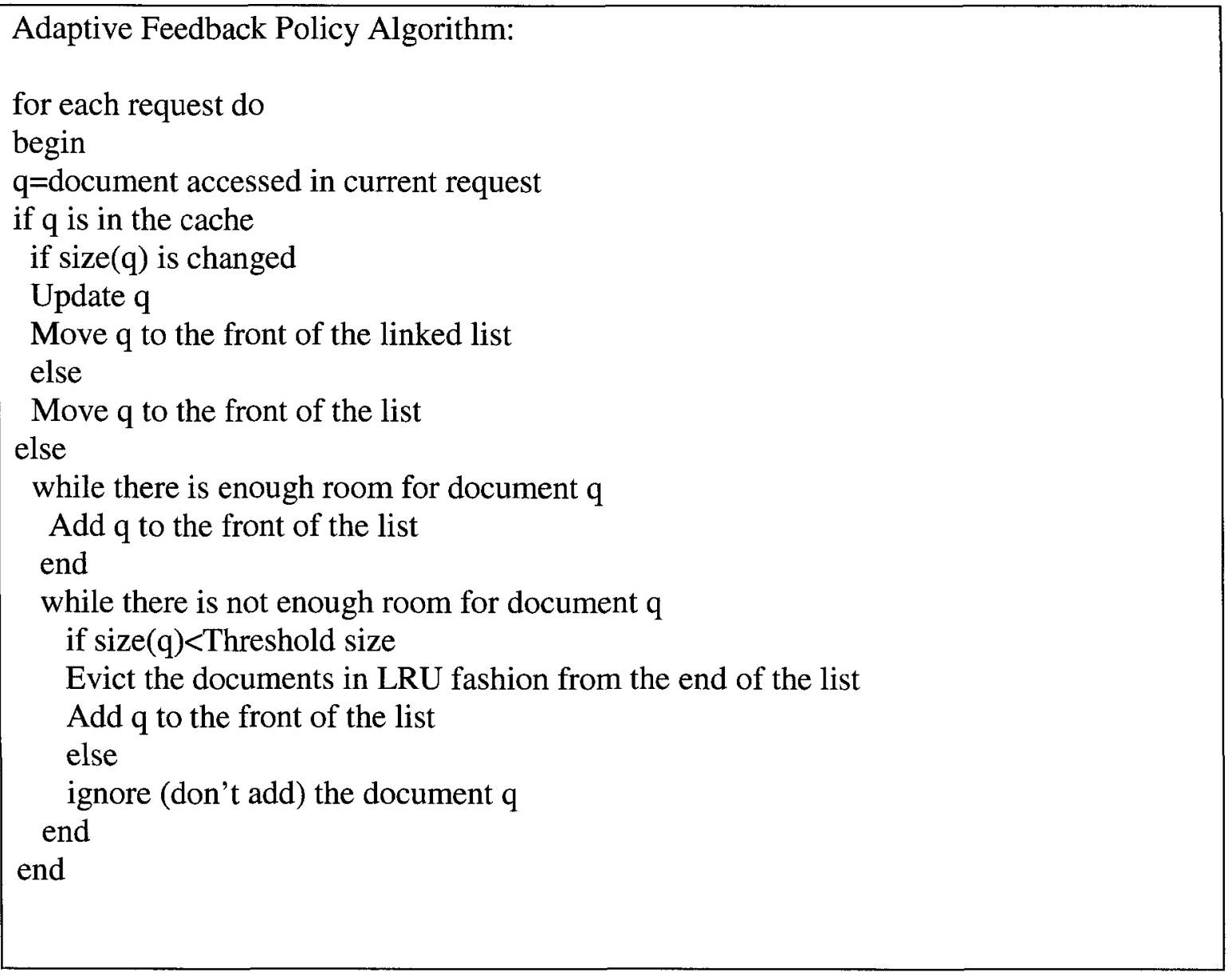

Figure 4.1: Adaptive Feedback Admission Control Policy Algorithm used by the Simulation Model

\subsection{Simulation and Results}

The adaptive feedback admission policy discussed in Section 4.3 has been implemented to analyze proxy cache performance using trace driven simulations. In order to compare the performance of the adaptive feedback policy against the standard LRU and the LRUThreshold policies, a trace driven cache simulator was developed in JAVA. For our study, we have used seven sanitized access logs obtained upon request from NLANR. Due to the extremely large data set that we used in our simulation study, it was necessary to 
implement the simulator efficiently. Thus our initial focus was to reduce the complexity of the actions performed by the simulator. The clean logs for each of the traces were first obtained by the data reduction techniques discussed in Section 3.2.2. These clean logs were then fed into the trace driven simulator that simulated a proxy cache of certain size.

An important aspect of any simulation study involves validating the simulator. We took several precautions to ensure that the simulator was behaving correctly. For example, the simulator was initially tested using very short traces (e.g. 50 requests) which could be verified manually. The results obtained from our simulator were repeatable. Two key parameters used in our simulation are the Cache Size and the Cache Management Policies. These are discussed in Sections 4.4.1 and 4.4.2 below.

\subsubsection{Cache Sizes}

The cache size indicates the amount of space available for storing Web documents. The number of distinct documents represents an upper bound on the number of documents that could be cached. The total size (bytes transferred) of these unique documents indicates the maximum useful (Infinite) cache size. For the seven traces considered in the analysis, the statistics in terms of distinct requests, size, maximum achievable hit ratio and byte hit ratio are given in Table 4.1 .

The maximum hit ratio and byte hit ratio values for each trace are obtained as follows:

$$
\begin{aligned}
& \text { Maximum Hit Ratio }=(\text { Total Requests }- \text { Distinct Requests }) / \text { Total requests } \\
& \text { Maximum Byte Hit Ratio }=(\text { Total Bytes }- \text { Infinite Cache Size }) / \text { Total Bytes }
\end{aligned}
$$




\begin{tabular}{|c|c|c|c|c|c|c|c|}
\hline Item & UC1 & UC2 & BO1 & BO2 & PA & PB & NY \\
\hline Total Requests & 609,196 & 534,585 & 352,309 & 273,982 & 263,032 & 240,769 & 160,431 \\
\hline $\begin{array}{c}\text { Total Bytes } \\
\text { (Mbytes) }\end{array}$ & 6865 & 6119 & 4613 & 4194 & 5269 & 3930 & 1800 \\
\hline $\begin{array}{c}\text { Distinct } \\
\text { Requests }\end{array}$ & 296,532 & 248,096 & 229,633 & 178,946 & 187,722 & 144,658 & 126,214 \\
\hline $\begin{array}{c}\text { Infinite Cache } \\
\text { Size (Mbytes) }\end{array}$ & 3748 & 3337 & 2722 & 2694 & 3199 & 1594 & 1464 \\
\hline $\begin{array}{c}\text { Maximum Hit } \\
\text { Ratio }\end{array}$ & 0.51 & 0.53 & 0.35 & 0.35 & 0.29 & 0.4 & 0.21 \\
\hline $\begin{array}{c}\text { Maximum Byte } \\
\text { Hit Ratio }\end{array}$ & 0.45 & 0.45 & 0.41 & 0.36 & 0.39 & 0.59 & 0.19 \\
\hline
\end{tabular}

Table 4.1: Statistics for seven NLANR traces

As shown in Table 4.1, the maximum hit ratio and byte hit ratio values for each trace are obtained for infinite cache size without incurring any document replacement. The maximum hit ratio achieved for the NLANR traces lies between 0.21 for the NY trace and 0.53 for the UC2 trace. The maximum byte hit ratio achieved for these traces lies between 0.19 for the NY trace and 0.59 for the PB trace. The wide variation in the maximum achievable hit ratio and byte hit ratio for the NLANR traces is due to trace dependent document referencing properties discussed in Chapter 3.

As noted in Table 3.3, the NY trace has the largest concentration (91\%) of one timer documents compared to the other traces and this influences the hit ratio observed in Table 4.1. The byte hit ratio for the PB trace is higher when compared to the UC2 trace because the popular documents requested for the PB trace are much larger in size. Due to the filtering effects of Web caching hierarchies, the maximum achievable hit ratio and byte hit ratio is lower for NLANR based top-level proxies compared to other lower level 
proxies such as institutional caches where hit ratios have been found to vary between $80 \%-90 \%$ [69].

No matter how large the cache size is, in the context of the Web, it is impossible and unnecessary to store all the documents so as to avoid cache pollution with inactive or valueless data. In practice the cache size is finite so that only a subset of requested documents can be stored. When the cache size is full, a suitably designed replacement policy is used to evict the documents based on a predetermined set of parameters such as their frequency, recency, size etc. [73]. As replacement policies are effective at lower cache sizes, it is important to evaluate the performance of any new policy against existing policies at lower cache sizes.

For our simulation study, a total of nine different levels were considered for the cache size parameter from 4 Mbytes to 1024 Mbytes, where each level was a factor of two larger than the previous size in the cache. A maximum cache size of 1024 Mbytes was considered as it constitutes $\sim 27 \%$ to $70 \%$ of the infinite cache size for all the traces. Any increase in the cache size beyond 1024 Mbytes would not yield noticeable improvement in the document hit ratio and the byte hit ratio. For lower cache size below 4 Mbytes, we have observed that the performance improvements using the LRU-AT were significantly higher compared to LRU and LRU-T policies. Thus by varying the cache size, the document hit ratio and byte hit ratio performance metrics are analyzed using the adaptive feedback policy and their performance compared against the standard replacement policies. 


\subsubsection{Cache Management Policies}

The second factor that we investigate in this simulation study is the comparison of cache replacement policies. In this simulation, the newly proposed adaptive feedback admission control policy discussed in Section 4.3 is compared with the standard LRU and LRUThreshold policies. To evaluate these three policies, a trace-driven simulation was performed on all seven NLANR traces discussed in Chapter 3.

The Least Recently Used (LRU) policy caches all documents which are below the cache size. When the cache is full, it removes the document which has not been accessed for the longest period of time. The LRU is a very simple policy requiring no parameterization. This policy works well for workloads which exhibit strong temporal locality.

The LRU-Threshold policy on the other hand caches only documents which are less than or equal to a fixed threshold size. Although it may sound simple, it is really a complicated trade-off to decide the value of the fixed threshold size. If the threshold is chosen too large, then the documents may end up thrashing for the same cache space and no document will remain in the cache for a duration long enough to achieve a good hit ratio. If the threshold is chosen too small, then the cache may be underutilized. Thus a fixed threshold may not always result in a decent hit ratio for a given trace.

The LRU-Threshold policy uses a threshold value which is a fixed percentage of the cache size. We have kept the threshold size constant for two reasons: First of all, it is difficult to estimate the optimal threshold value for different traces and for different 
cache sizes. Secondly if we have different threshold values for different traces it is hard to compare the results.

In order to overcome the cache performance limitations posed by the LRU and the LRUT policies, LRU-AT policy adaptively tunes the initial threshold value by monitoring the hit ratio as discussed in Section 4.3.

\subsubsection{Results}

This Section provides the simulation results based on our study of three proxy cache replacement policies [1]. The cache performance for the three policies is examined in terms of the document hit ratio and the byte hit ratio. In Figures 4.2 to 4.8 , the document hit ratio is shown as a function of varying the cache size for the three replacement policies. In Figures 4.2 to 4.15, the legend LRU refers to the standard LRU policy, LRUAT refers to the adaptive threshold policy and LRU-T refers to the LRU-Threshold policy.

Figures 4.2 and 4.3 show the hit ratio as a function of cache size for the UC1 and the UC2 trace respectively. These two traces are from the same proxy (UC-Urbana Champaign) taken on two consecutive days. The maximum cache size of 1024 Mbytes corresponds to $27 \%$ and $30 \%$ of infinite cache size for the UC1 and UC2 traces. The maximum hit ratios achievable for infinite cache size for these two traces are $51 \%$ and $53 \%$ respectively. 
As seen in Figures 4.2 and 4.3, LRU-AT performs well above the standard LRU for cache sizes from 4 Mbytes to 1024 Mbytes. It should also be noted that the performance of the LRU-AT against the LRU-T is improved for cache sizes from 4 Mbytes to $\sim 256$ Mbytes. Beyond 256 Mbytes, LRU-T outperforms LRU-AT and LRU policies for both traces as the threshold approaches the optimal value. For a cache size of 4 Mbytes, the document hit ratio in both the $\mathrm{UC} 1$ and the $\mathrm{UC2}$ traces with the LRU-AT policy is improved by $4.15 \%$ to $5.40 \%$ over the standard LRU policy, and by $6.79 \%$ to $9.84 \%$ over the LRU-T policy.

Figures 4.4 and 4.5 show the hit ratio as a function of cache size for the $\mathrm{BO} 1$ and $\mathrm{BO} 2$ traces respectively. These two traces are from the same proxy (BO-Boulder) taken on two consecutive days. The maximum cache size of 1024 Mbytes corresponds to $37 \%$ and $38 \%$ of the infinite cache size for the $\mathrm{BO} 1$ and $\mathrm{BO} 2$ traces. The maximum hit ratio achievable for infinite cache size for these two traces is $35 \%$.

As seen in Figures 4.4 and 4.5, LRU-AT again performs well above the LRU and LRU-T for cache sizes from 4 Mbytes to 16 Mbytes. For a cache size of $4 \mathrm{Mbytes}$, the document hit ratio in both $\mathrm{BO} 1$ and $\mathrm{BO} 2$ traces with LRU-AT policy is improved by $4.32 \%$ to $4.89 \%$ over the standard LRU policy and by $4.77 \%$ to $6.44 \%$ over the LRU-T policy. Beyond a cache size of 16 Mbytes, the LRU-AT and LRU-T policies are almost identical, and outperform LRU policy for both traces.

Figures 4.6, 4.7 and 4.8 show the hit ratio as a function of cache size for PA, PB and NY traces respectively. These three traces are taken from three different proxies (PA, PB and NY) in NLANR. The maximum cache size of 1024 Mbytes corresponds to $32 \%, 64 \%$ and 
$70 \%$ of infinite cache size for $\mathrm{PA}, \mathrm{PB}$ and $\mathrm{NY}$ traces respectively. The maximum hit ratio achievable for the infinite cache size for these three traces is $29 \%$ (PA), $40 \%$ (PB) and $21 \%(\mathrm{NY})$ respectively.

Again, from Figures 4.6, 4.7 and 4.8, we see that the LRU-AT outperforms the LRU and the LRU-T for cache sizes from 4 Mbytes to 1024 Mbytes. For a cache size of 4 Mbytes, the document hit ratio in the PA, PB and NY traces with LRU-AT policy is improved by $1.98 \%$ to $2.68 \%$ over the standard LRU policy and $1.71 \%$ to $6.07 \%$ over the LRU-T policy. A cache size of 4 Mbytes for the PB and NY trace corresponds to $25 \%$ and $27 \%$ of the infinite cache size, whereas for the PA trace it amounts to only $12.5 \%$. Thus for cache sizes lower than 4 Mbytes, the performance improvement obtained with LRU-AT policy for the PB and NY traces will be much higher when compared to the LRU and LRU-T policies.

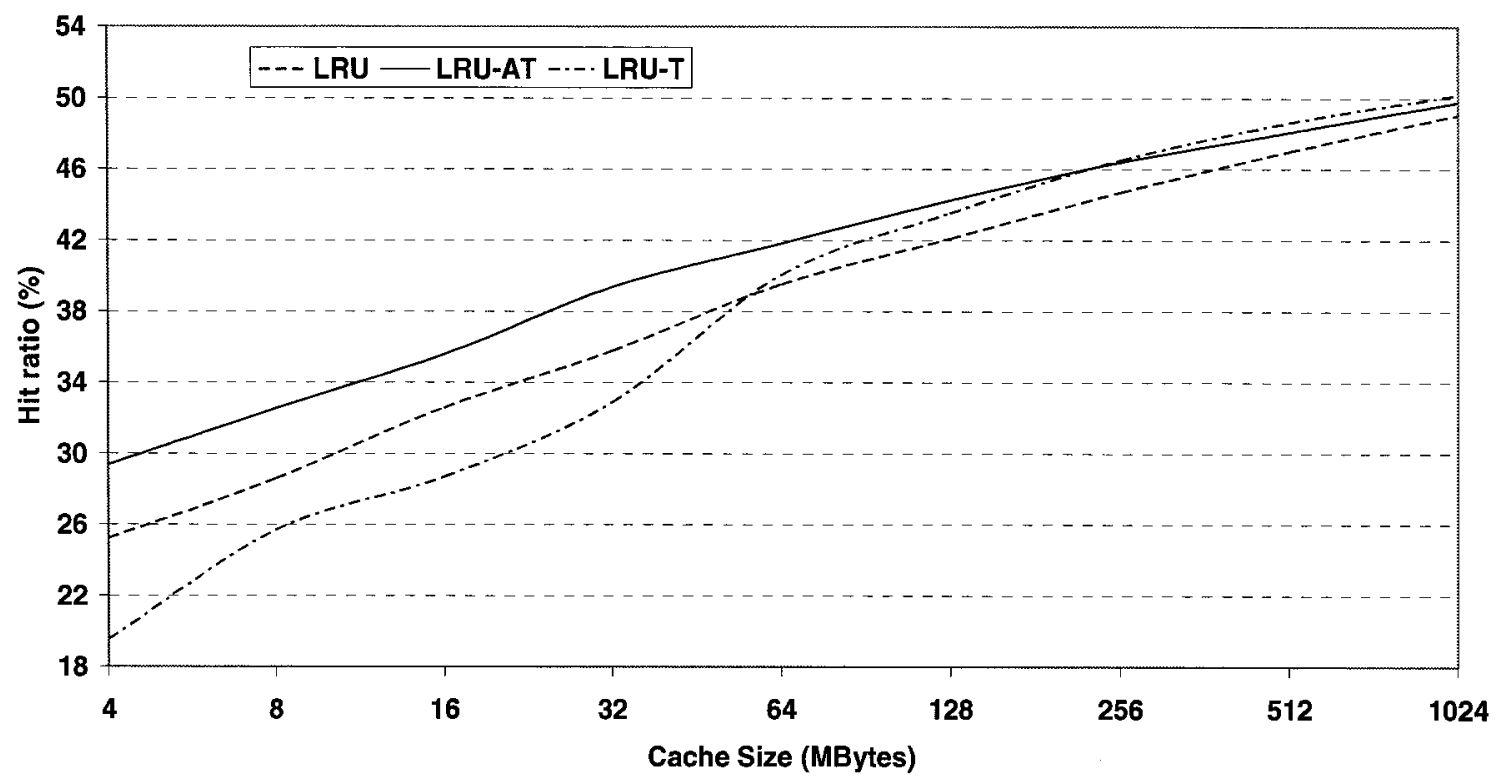

Figure 4.2: Hit ratio vs Cache size for UC1 trace 


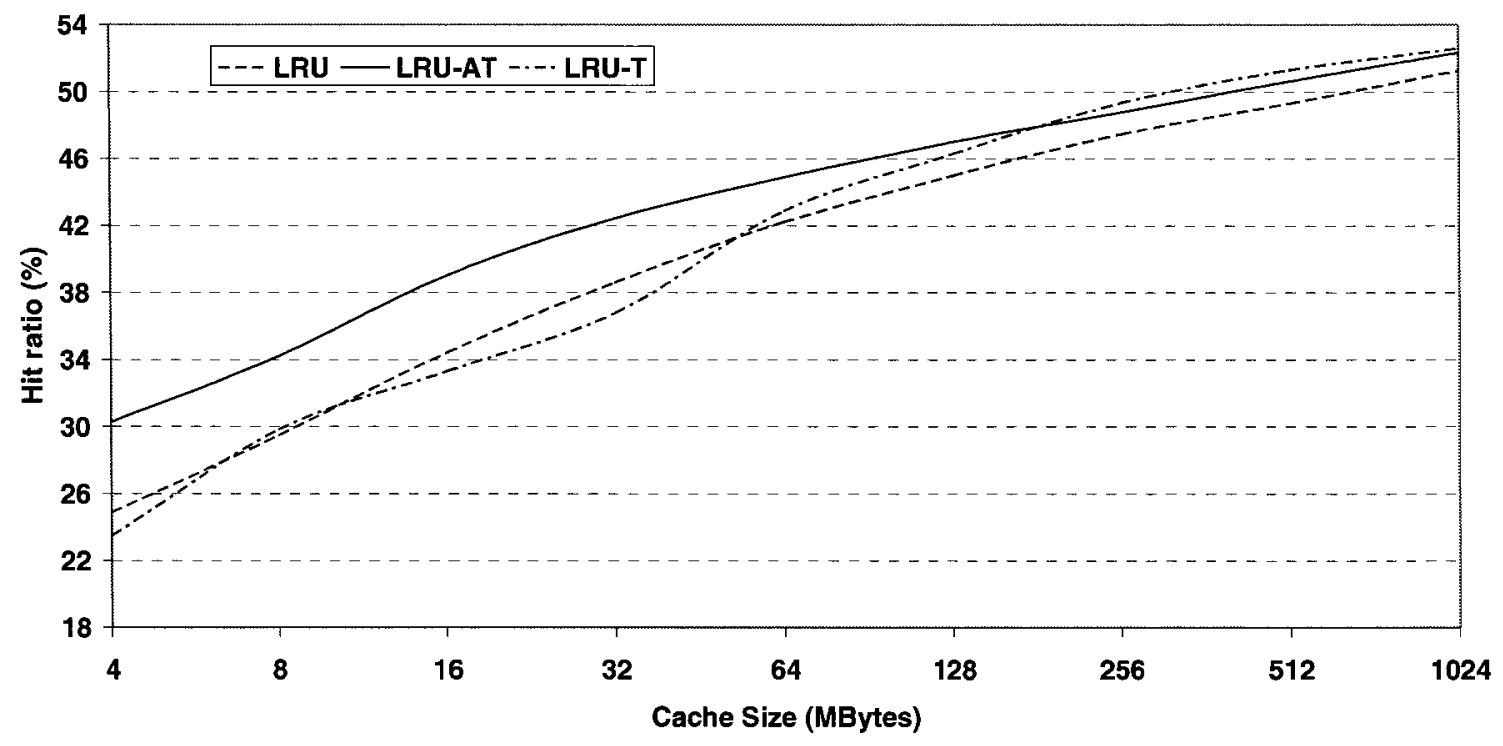

Figure 4.3: Hit ratio vs Cache size for UC2 trace

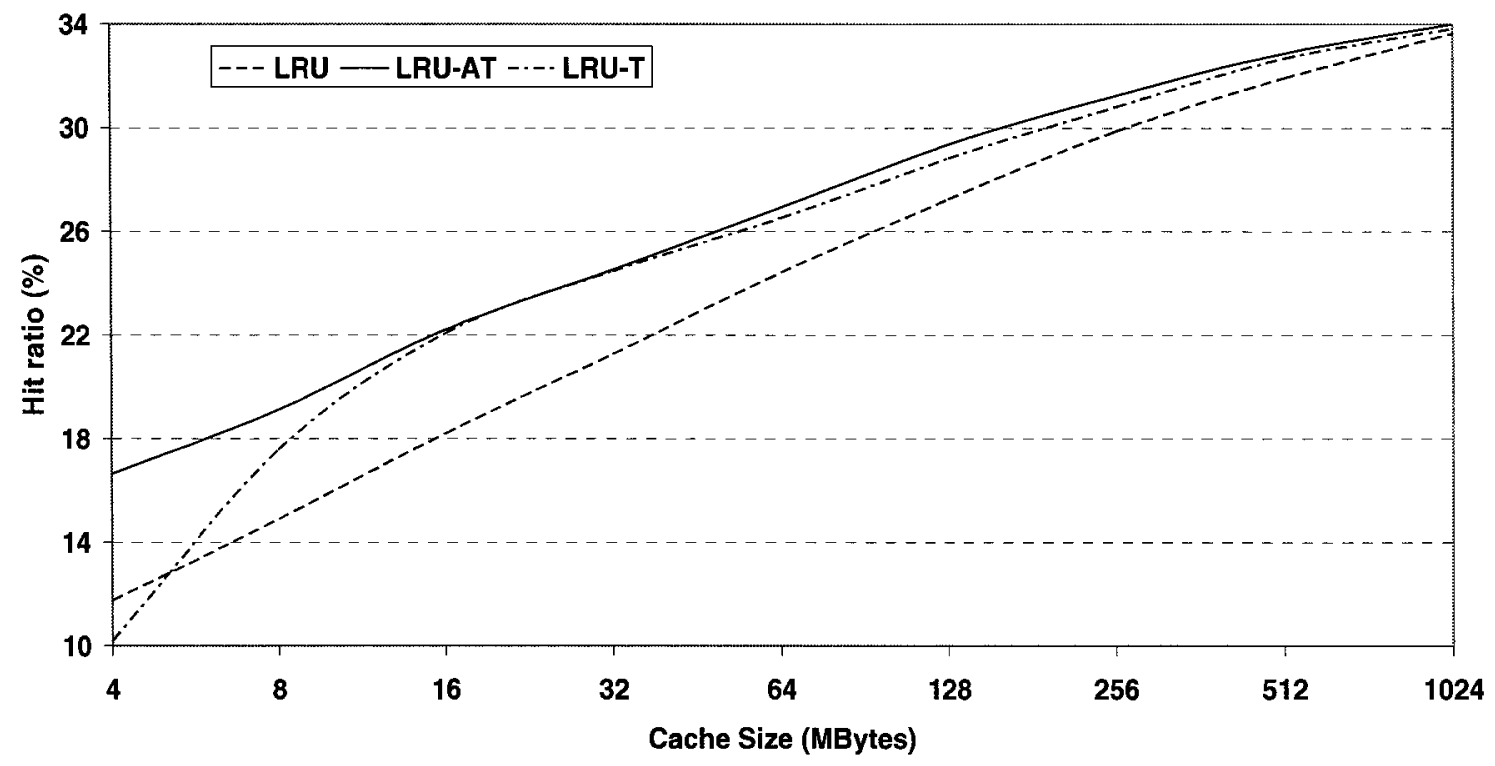

Figure 4.4: Hit ratio vs Cache size for $\mathrm{BO} 1$ trace 


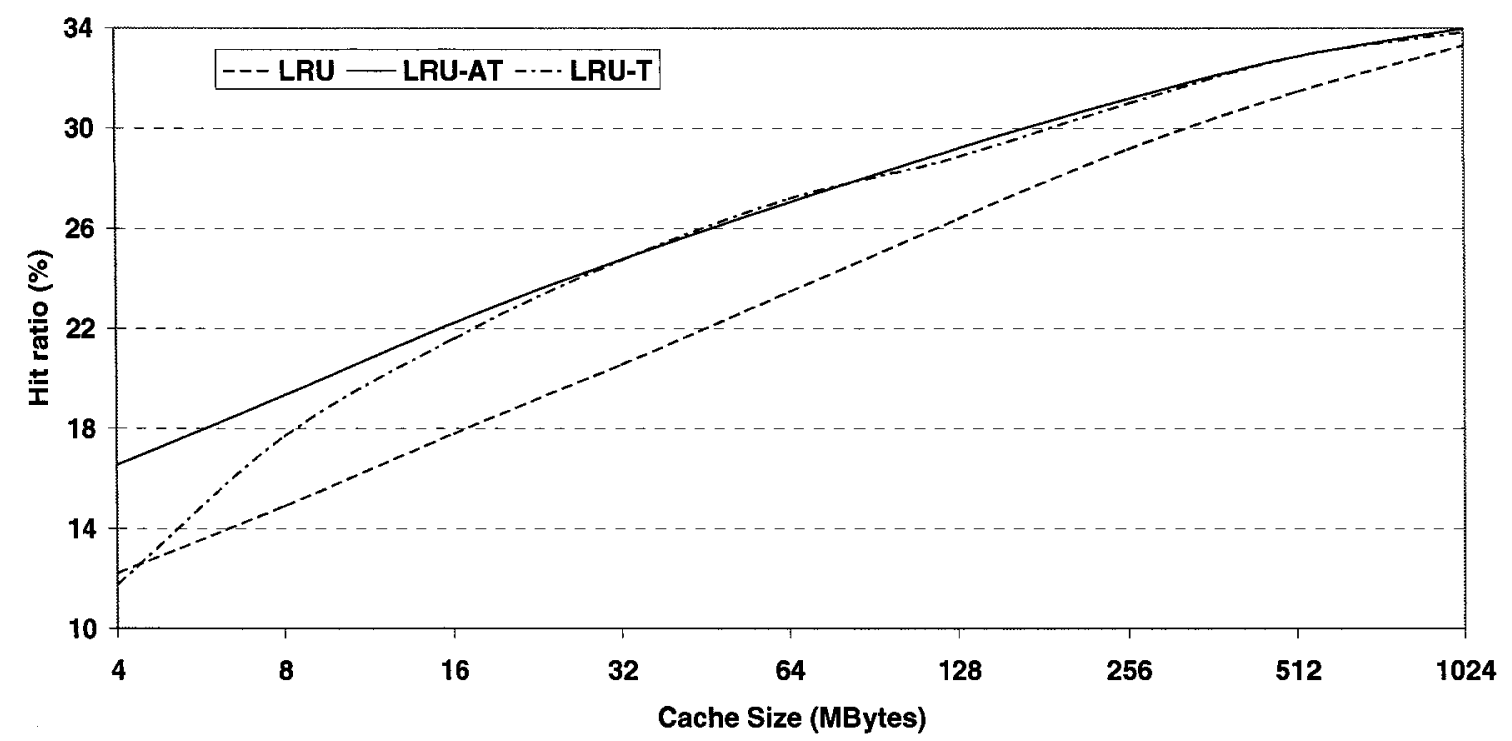

Figure 4.5: Hit ratio vs Cache size for $\mathrm{BO} 2$ trace

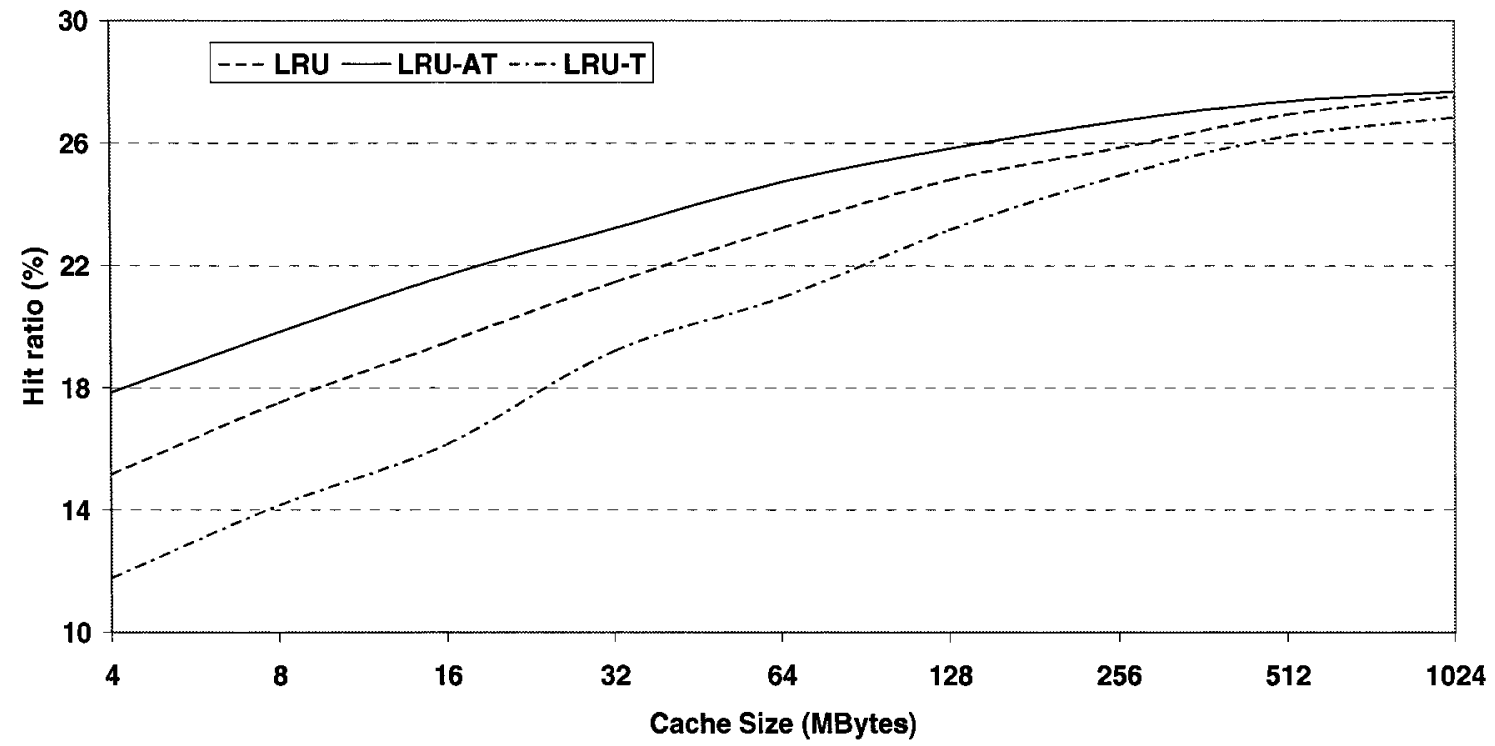

Figure 4.6: Hit ratio vs Cache size for PA trace 


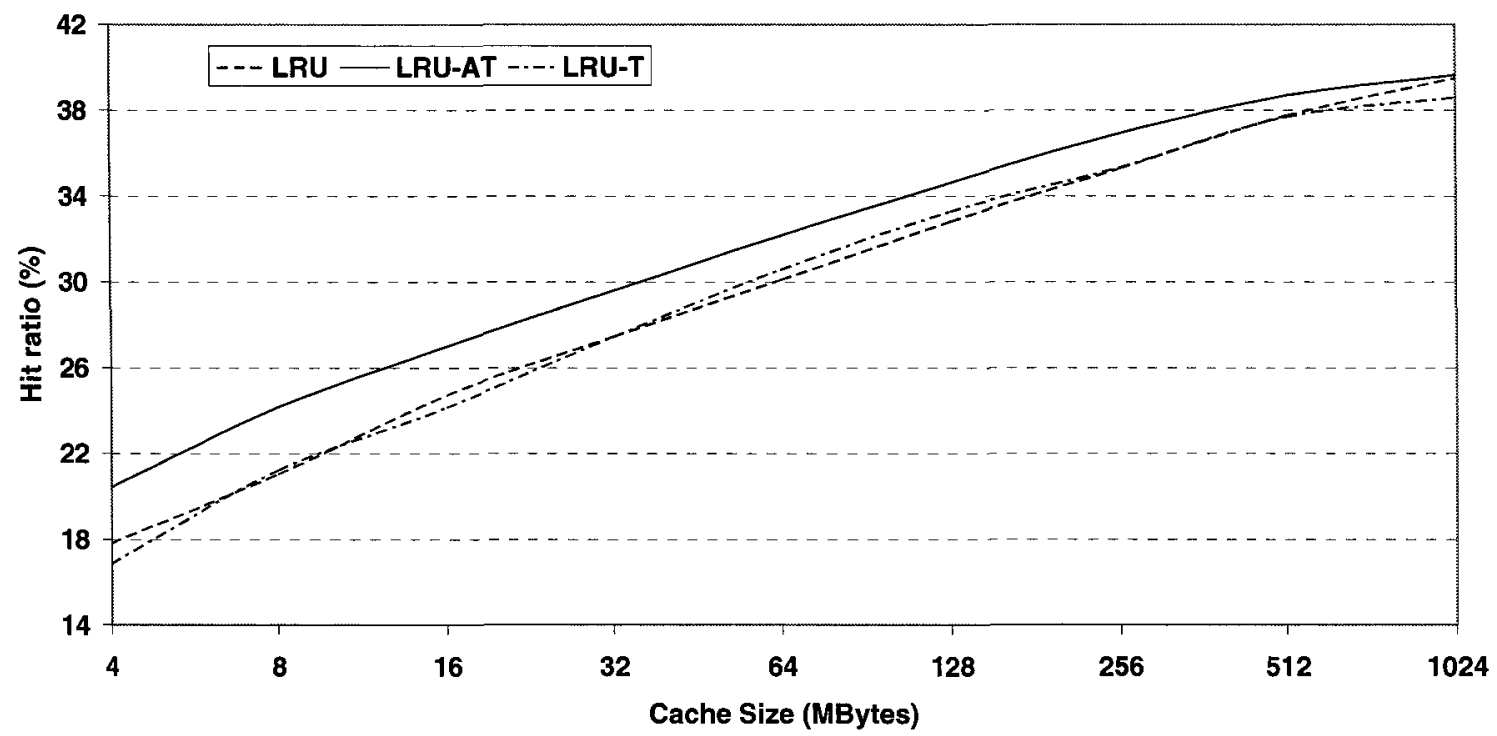

Figure 4.7: Hit ratio vs Cache size for PB trace

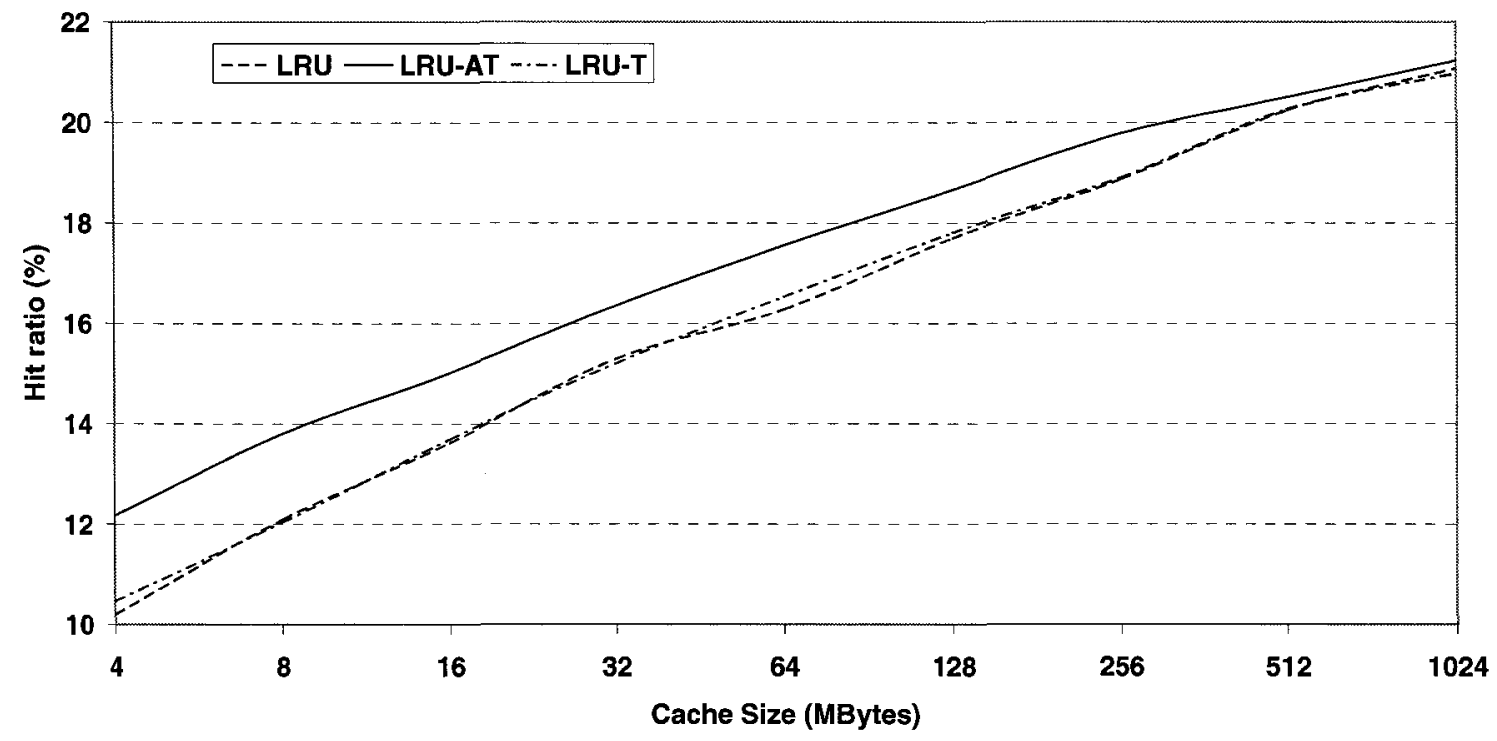

Figure 4.8: Hit ratio vs Cache size for NY trace 
It can be seen from Figures 4.2 to 4.8 that the LRU-AT policy provides an improvement in the document hit ratios at lower cache sizes, when compared to standard LRU and LRU-T policies. For a cache size of 4 Mbytes, the UC trace achieved the highest improvement of $\sim 10 \%$ in hit ratio with the LRU-AT policy when compared to the LRU-T policy, whereas an improvement greater than $5 \%$ is achieved over the standard LRU policy. With the LRU-AT policy, the varying performance improvements obtained in the document hit ratio as a function of the cache size is heavily dependent on the trace properties within a given proxy.

For the seven traces considered in this study, we have shown that the document hit ratio could be improved by $\sim 5 \%$ to $10 \%$ at a cache size of 4 Mbytes. For cache size below 4 Mbytes, we notice that the adaptive threshold tuning policy achieves significantly better performance compared to the LRU and LRU-T policies. The nature of this improvement is due to the NLANR trace properties, which are obtained from a higher-level proxy cache. The objective of any cache replacement policy is to maximize the hit ratios for a given cache size so as to improve the user response time and reduce the latency. Thus, using the LRU-AT policy for optimizing cache performance measured in terms of the hit ratios at lower cache sizes could improve the user response time and reduce the latency.

Based on our experiments, we believe that we can draw the following generic conclusions from Figures 4.2 to 4.8:

1. LRU-AT policy performs well at lower cache sizes compared to the LRU-T and LRU policies. In order to validate the effect of a replacement policy, it is necessary to test the policy at lower cache sizes where the frequency of 
replacements is high. By imposing admission constraints in conjunction with a replacement strategy, the net effect is reflected as an increase in the document hit ratio at lower cache sizes.

2. At higher cache sizes, we notice that all the three policies considered in our experiments seem to converge in terms of the hit ratio. As the cache size becomes arbitrarily large for a given trace, there are very few replacements, and obviously, imposing any further static or dynamic admission constraints will not improve the hit ratio.

3. We also notice that the LRU-T policy outperforms the LRU-AT by a small margin for certain cache sizes in some of the traces. Due to the transfer size characteristics associated with such traces, the fixed size threshold considered for the LRU-T policy at a given cache size may be optimal, which could be the cause of this improved hit ratio.

\subsubsection{Byte hit ratio}

The byte hit ratio refers to the ratio of data transferred from the cache to the total data requested, and is defined in Section 4.2.2. A higher byte hit ratio results in reduced traffic transported in the core network leading to lesser congestion and transport cost savings. Earlier studies have shown that policies that use size as an admission constraint, such as LRU-T, discriminate against large documents resulting in low byte hit ratio [32]. In order to achieve a higher byte hit ratios, large documents should be cached which in turn requires really large caches. Caching large documents in small caches will evict smaller 
size documents that are more frequently used, and this will have a reverse impact on the hit ratio.

In our study, the cache performance is evaluated by examining the document byte hit ratio as a function of cache size. All the three policies used in evaluating the document hit ratio are used here to analyze the document byte hit ratio. Figures 4.9 to 4.15 show the byte hit ratio as a function of varying cache size for the seven NLANR traces.

Figures 4.9 and 4.10 show the byte hit ratio as a function of cache size for UC1 and UC2 trace respectively. As mentioned, these two traces are from the same proxy (UC-Urbana Champaign) taken on two consecutive days. The maximum cache size of 1024 Mbytes corresponds to $27 \%$ and $30 \%$ of the infinite cache sizes for the UC1 and UC2 traces. The maximum byte hit ratio achievable for infinite cache size for these two traces is $45 \%$ and this is achieved at $1024 \mathrm{MB}$.

As seen in Figures 4.9 and 4.10, both LRU-AT and LRU policies perform well above LRU-T for cache sizes from 4 Mbytes to 1024 Mbytes. For the lowest cache size of 4 Mbytes, the document byte hit ratio in both the UC1 and UC2 traces with the LRU-AT policy is improved by $14.6 \%$ to $20 \%$ over the LRU-T policy. At the same cache size, the standard LRU policy improves the byte hit ratio by $19 \%$ to $21.5 \%$ over the LRU-T policy. For the largest cache size of 1024 Mbytes, the document byte hit ratio in both the UC1 and UC2 traces with the LRU-AT policy is improved by $26 \%$ to $28 \%$ over the LRU-T policy. At the same cache size, the standard LRU policy improves the byte hit ratio by $25 \%$ to $30 \%$ over the LRU-T policy. 
Figures 4.11 and 4.12 show the byte hit ratio as a function of cache size for the BO1 and $\mathrm{BO} 2$ trace respectively. These two traces are from the same proxy (BO-Boulder) taken on two consecutive days. The maximum cache size of 1024 Mbytes corresponds to $37 \%$ and $38 \%$ of infinite cache size for the $\mathrm{BO} 1$ and $\mathrm{BO} 2$ traces. The maximum byte hit ratio achievable for infinite cache size for these two traces are $41 \%$ and $36 \%$ respectively.

As seen in Figures 4.11 and 4.12, the LRU-AT and LRU policies perform well above the LRU-T policy for cache sizes from 4 Mbytes to 1024 Mbytes. For the lowest cache size of 4 Mbytes, the document byte hit ratio in both the BO1 and BO2 traces with the LRUAT policy is improved by $6 \%$ to $7 \%$ over the LRU-T policy. At the same cache size, the standard LRU policy improves the byte hit ratio by $15 \%$ to $17 \%$ over the LRU-T policy. For the largest cache size of 1024 Mbytes, the document byte hit ratio in both the UC1 and the UC2 traces with LRU-AT policy is improved by $27.5 \%$ to $29.8 \%$ over the LRU-T policy. At the same cache size, the standard LRU policy improves byte hit ratio by $28.7 \%$ to $30.7 \%$ over the LRU-T policy.

Figures $4.13,4.14$ and 4.15 show the byte hit ratio as a function of cache size for PA, PB and NY traces respectively. These three traces are taken from three different proxies (PA, PB and NY) in NLANR. The maximum cache size of 1024 Mbytes corresponds to 32\%, $64 \%$ and $70 \%$ of the infinite cache sizes for the PA, PB and NY traces. The maximum byte hit ratio achievable for infinite cache sizes for these three traces is $39 \%$ (PA), 59\% (PB) and $19 \%(\mathrm{NY})$.

As seen in Figures 4.13, 4.14 and 4.15, the LRU-AT and LRU outperform the LRU-T policy for cache sizes from 4 Mbytes to 1024 Mbytes. For the lowest cache size of 4 
Mbytes, the document byte hit ratio in the PA, PB and NY traces with the LRU-AT policy is improved by $3 \%$ to $42 \%$ over the LRU-T policy. At the same cache size, standard LRU policy improves byte hit ratio by $6.6 \%$ to $44 \%$ over the LRU-T policy. For the largest cache size of 1024 Mbytes, the document byte hit ratio in the PA, PB and NY traces with the LRU-AT policy is improved by $11.5 \%$ to $43 \%$ over the LRU-T policy. At the same cache size, the standard LRU policy improves the byte hit ratio by $11.6 \%$ to $50 \%$ over the LRU-T policy.

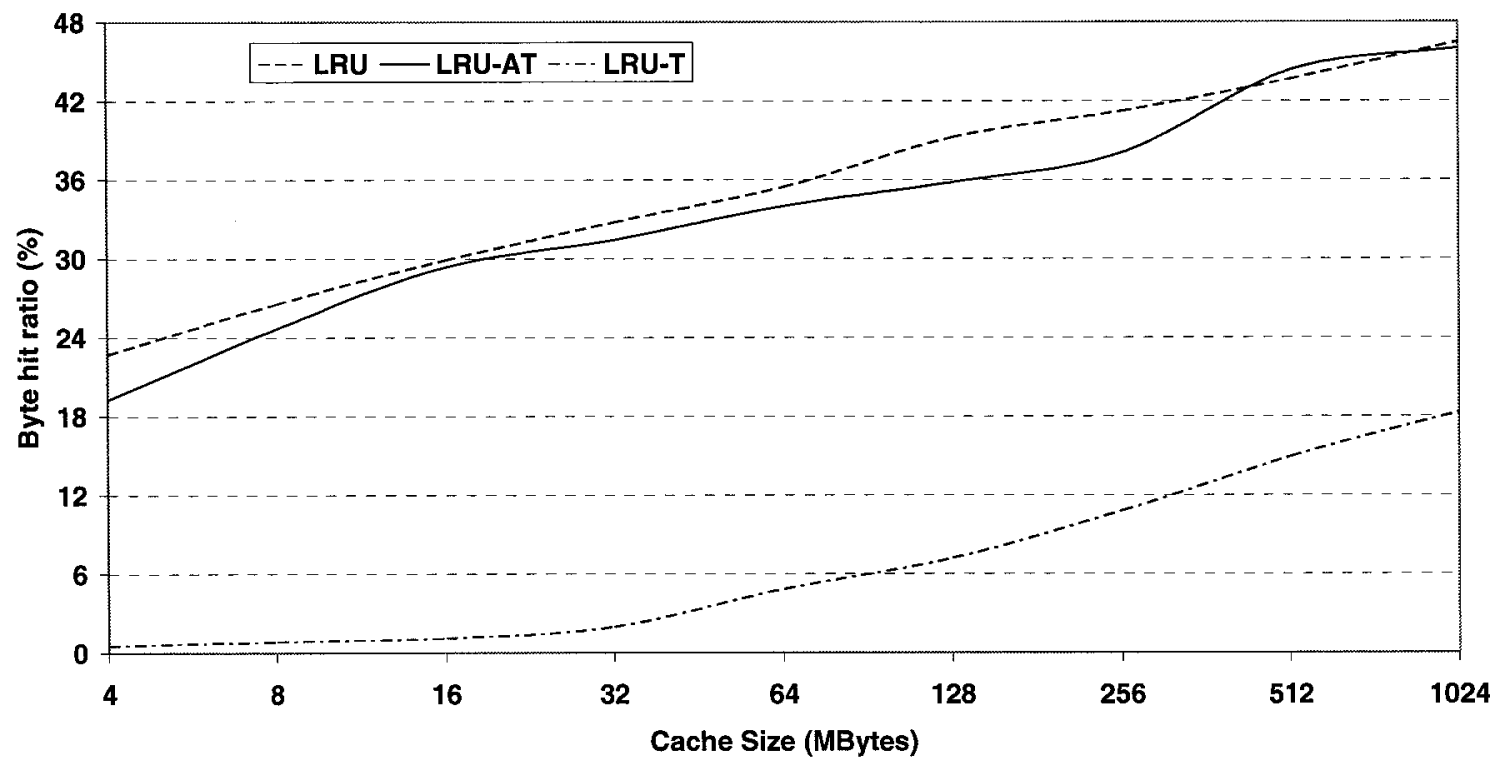

Figure 4.9: Byte hit ratio vs Cache size for UC1 trace 


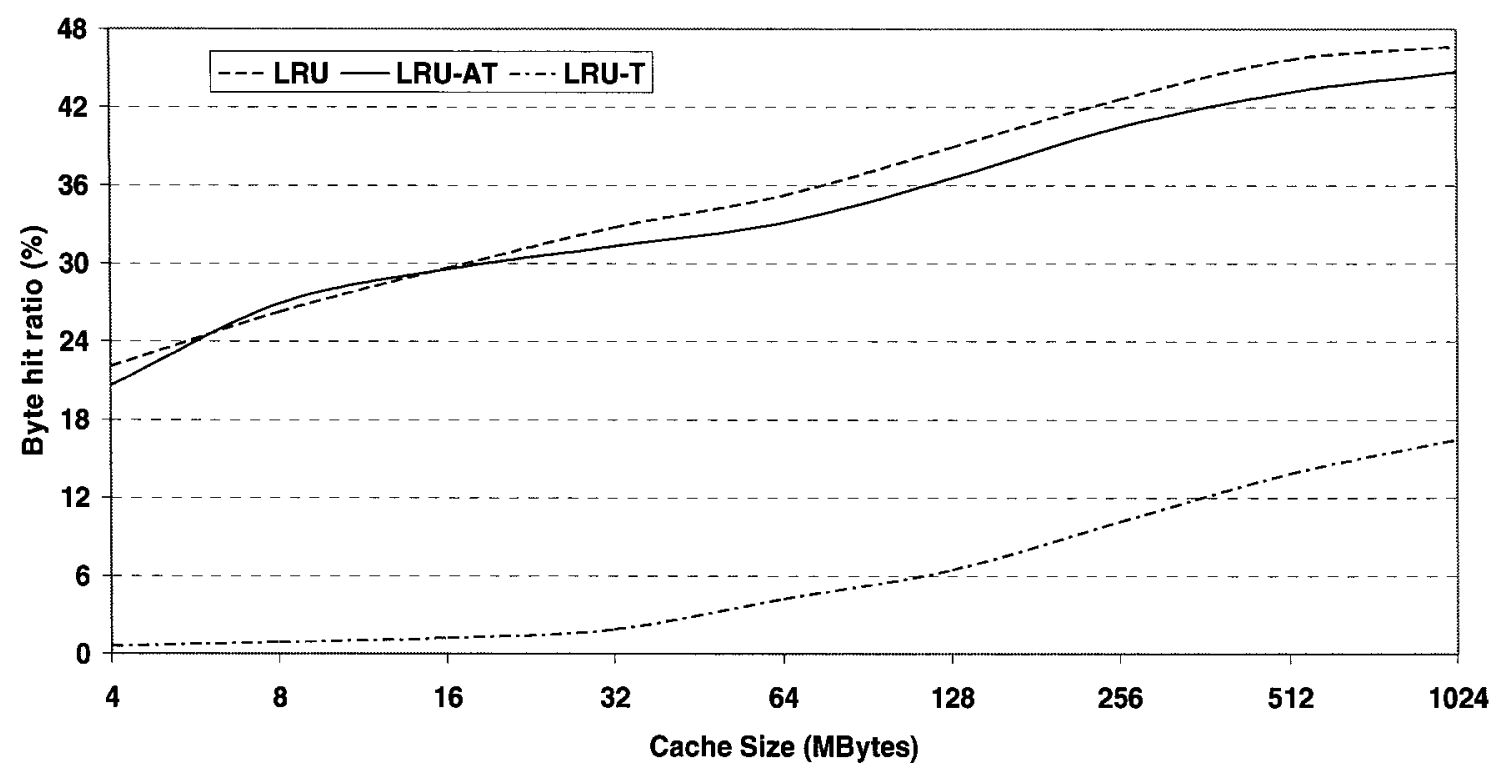

Figure 4.10: Byte hit ratio vs Cache size for UC2 trace

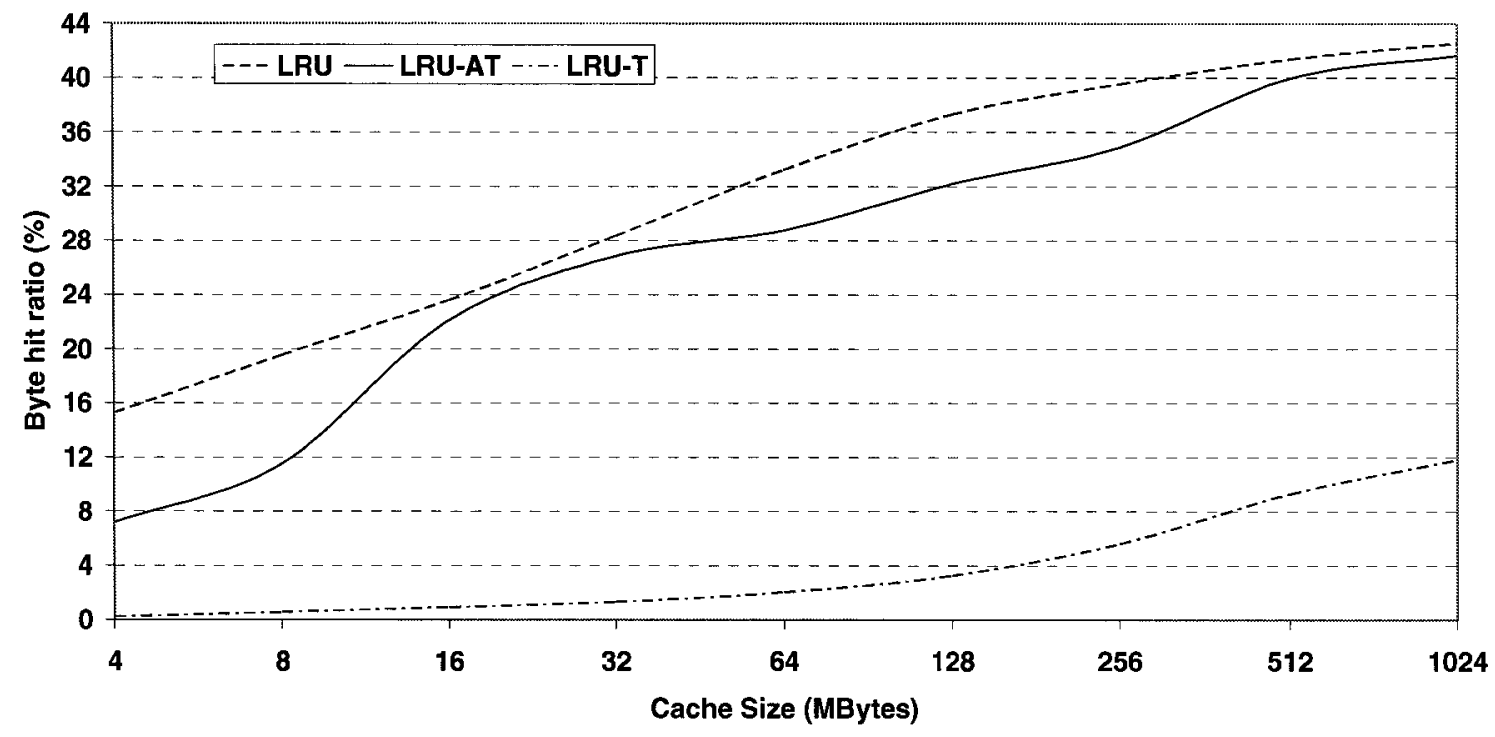

Figure 4.11: Byte hit ratio vs Cache size for BO1 trace 


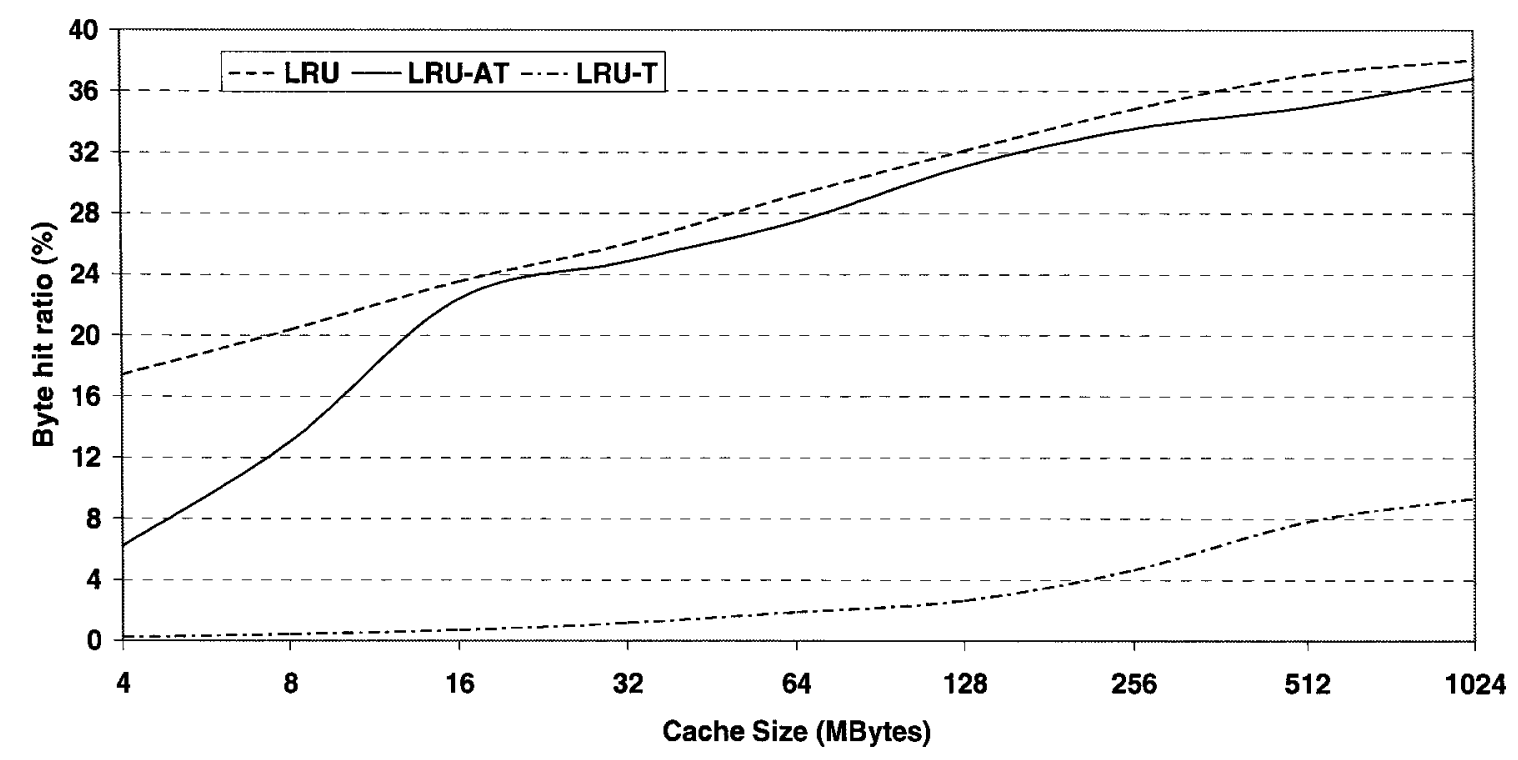

Figure 4.12: Byte hit ratio vs Cache size for BO2 trace

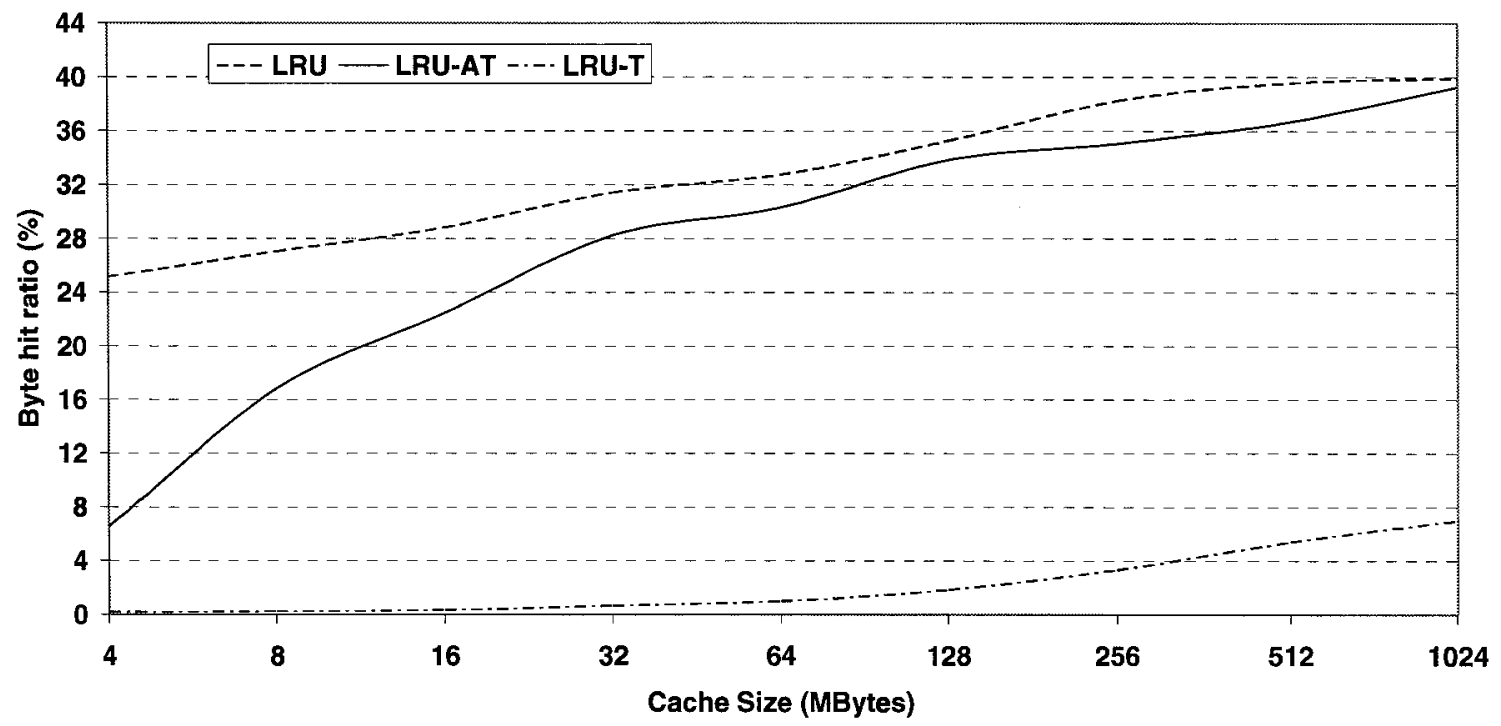

Figure 4.13: Byte hit ratio vs Cache size for PA trace 


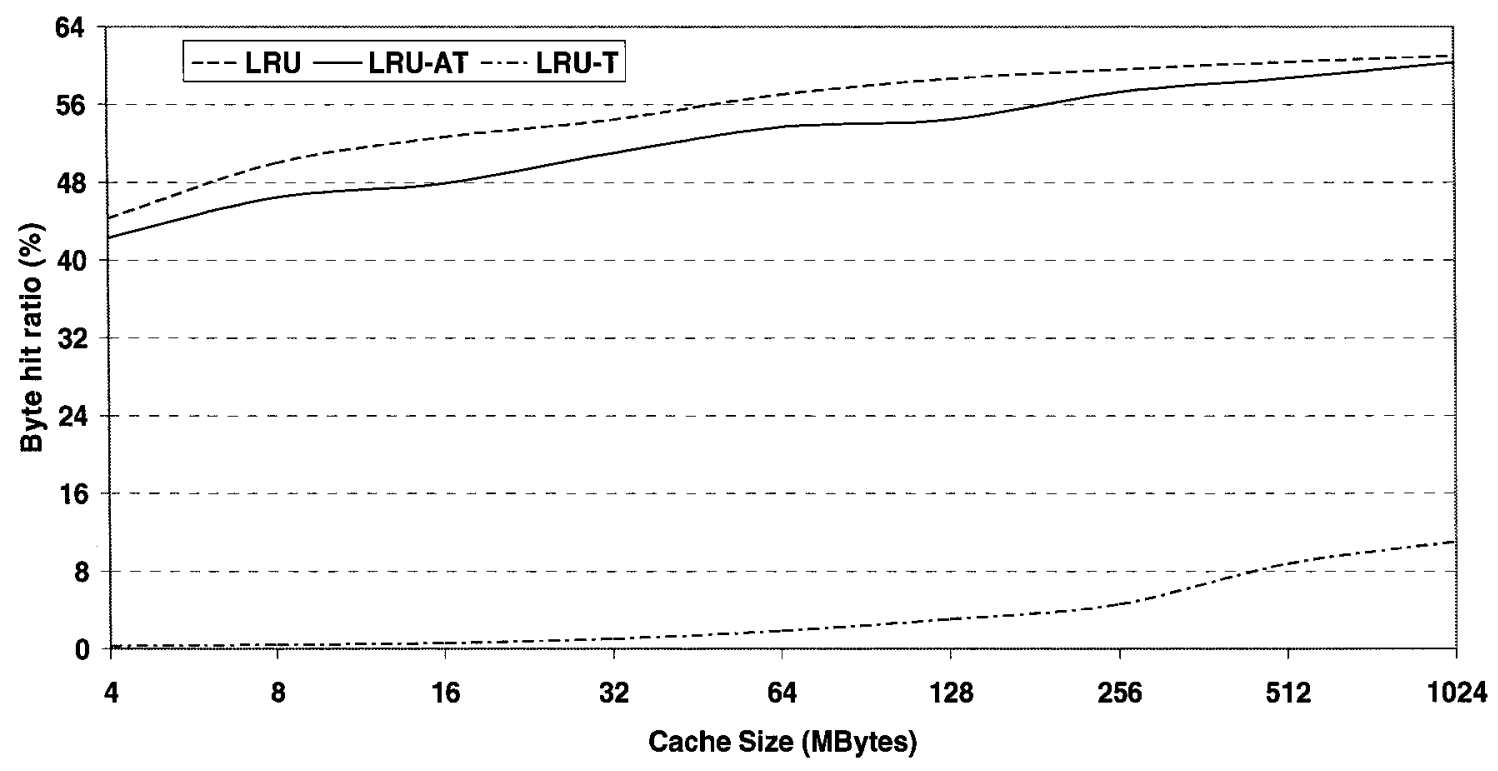

Figure 4.14: Byte hit ratio vs Cache size for PB trace

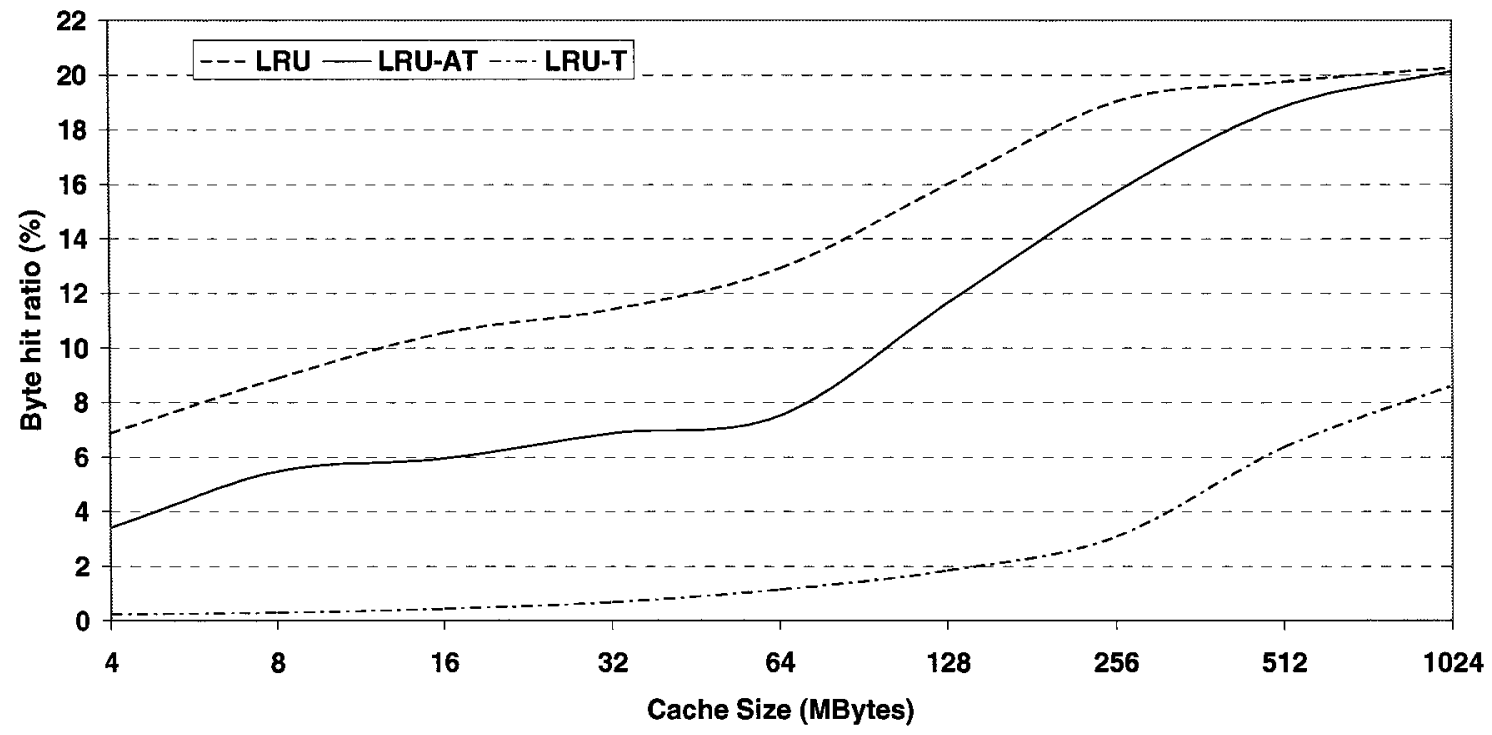

Figure 4.15: Byte hit ratio vs Cache size for NY trace 
It can be seen from Figures 4.9 to 4.15 that for all the NLANR traces, the LRU policy achieves the maximum byte hit ratio compared to the size-based policies. As the cache size increases, the byte hit ratio also increases for all the three policies whereas the maximum achievable byte hit ratio for a given trace is obtained with the LRU and the LRU-AT policies only. By adaptively tuning the admission constraint, the LRU-AT policy is able to cache documents more effectively compared to the LRU-T and, in turn, maximize the byte hit ratio.

As seen in Figures 4.9, 4.10 and 4.14, the byte hit ratio with the LRU-AT policy is comparable to the LRU for UC and PB proxies. It can also be seen from Figures 4.11, 4.12 and 4.13 that for traces within $\mathrm{BO}$ and PA proxies, there is a higher degree of variation in the byte hit ratio at lower cache sizes with the LRU-AT policy. As the cache size increases beyond $16 \mathrm{MB}$, the byte hit ratios achieved with the LRU-AT policy are comparable to the LRU policy. For the NY trace as shown in Figure 4.15, the variation in the byte hit ratio is much higher with the LRU-AT policy, and this is due to the size of the cached documents which range from a few kilobytes to a few megabytes. Depending on the trace properties in a given proxy, the LRU-AT policy is able to achieve byte hit ratios comparable to the LRU and, thus outperform the LRU-T policy.

The following generic conclusions can be drawn from Figures 4.9 to 4.15 based on our experiments:

As the LRU-AT policy uses the document size as an adaptive admission constraint, this will have a negative impact on byte hit ratio performance. We believe that the wide variation in the byte hit ratio is due to the adaptive tuning of the size filter. However, 
unlike LRU-AT policy, the static size threshold chosen for the LRU-T policy results in a lower byte hit ratio. Otherwise, we believe that it is premature to determine any additional conclusions based on the results that we have obtained.

\subsection{Summary}

This Chapter has proposed an adaptive feedback admission control policy to evaluate cache performance. The cache performance has been examined in terms of the document hit ratio and the byte hit ratio metrics. Using trace-driven simulations, we observe that for the seven traces considered from five different proxies within NLANR, the document hit ratio is improved using the LRU-AT policy. For the lowest cache size of 4 Mbytes considered in our analysis, an improvement of $\sim 5 \%$ to $10 \%$ in hit ratio was achieved with the LRU-AT policy over the LRU and the LRU-T policies. For lower cache sizes below 4 Mbytes, we have noticed that the LRU-AT policy yields greater than $10 \%$ improvement in hit ratio compared to the LRU and LRU-T policies.

When looking at the byte hit ratio performance metric at lower cache sizes, we find a reverse impact using the LRU-AT policy, and this is due to the size-based admission constraints discussed earlier in Section 4.4.3.1. At higher cache sizes, the LRU-AT policy is able to yield a byte hit ratio comparable to the LRU.

Williamson [71] has reported experiments involving Web characterization and caching, in which the data involved months of traffic. Although our data involves only "days" of traffic, we believe that our results and the corresponding conclusions arrived at, are still valid. 
For emerging applications like Voice over IP, Video Streaming and Video Telephony in Wireless Networks, that demand tighter specifications on the end-user response time and latency, we believe that the document hit ratio and byte hit ratio are critical cache performance metrics, where it appears, as if the former is more significant. It is essential to learn such changes in Wireless user's behaviors or access patterns and incorporate these changes in designing appropriate replacement policies so as to achieve better performance. 


\section{Caching Using Automata-based Partitioning}

\subsection{Introduction}

In Chapter 4 of this Thesis, we had presented a summary of the cache-performance of algorithms which are based on adaptive admission and replacement policies. By exploiting the temporal locality characteristics in web caching, and using adaptive admission constraints, we made an attempt to improve the cache performance in terms of the hit ratio and the byte hit ratio.

The LRU policy was used to perform the replacement of the documents in the cache. The time complexity of the LRU policy is the same as that of a linear search algorithm, nearly $O(\mathrm{n})$. In this policy the documents are arranged in terms of their descending order of their respective access frequency. As such, access frequencies are rarely known in advance, and so one has to resort to a heuristic to approximate an optimal behavior. In addition to the hit ratio and byte hit ratio metrics, the cache performance can also be examined in terms of the search cost of the requested documents.

By managing the content of the cache we can reduce the search cost. Additionally, we can show that with the help of automaton-based partitioning algorithms, it is possible to accurately classify the contents of the cache [74]. This Chapter examines the problem of self-organizing linear search lists as applied to web caching, and presents a list organization framework based on the newly proposed concept of Lists-on-Lists to potentially optimize the search cost. 
The long range objective of this Thesis was modified after the unfortunate demise of Professor Sivarama Dandamudi. Whereas the work under Professor Dandamudi involved the core traditional migration algorithms, after his demise, Professor Oommen supervised me and we decided to further consider the option of not only considering the effects of the reorganization but also of incorporating a learning mechanism into the whole paradigm. The intention was that we would be able to learn how the objects (files) cluster together by virtue of their similarity/joint access patterns. This Chapter represents the work we have done in this regard. Due to time constraints caused by the tragic events, a full implementation of this work was not possible.

Thus the work we mention in this Chapter represents the research that has transpired at the conceptual level. The implementation details will be left for a subsequent project.

\subsection{Adaptive Linear Lists}

In order to retrieve data from a list of $n$ records, a linear or sequential search is generally performed, with a time complexity of $O(\mathrm{n})$. The best ordering of the records would be in terms of the descending order of their respective access frequencies. Since we do not have prior knowledge of the record access frequencies, one has to resort to a heuristic to approximate an optimal behavior. If the contents of the cache can be dynamically adapted to the incoming requests using a self-organizing heuristic, it is potentially possible to minimize the search cost. A self-organizing linear search list is a list that runs a reorganization heuristic with each access query, with the hope of optimizing the linear search cost. 
Much of the early work on self-organizing linear search lists began with McCabe's research in 1965 [75]. He introduced two heuristics: the move-to-front rule (MTF), which moves the queried record to the front of the list, and the transposition rule (TR), which swaps the queried record with its predecessor. The MTF is characterized by quick convergence rates and the ability to quickly adapt to changes in the environment. The TR on the other hand, is more likely to find a better asymptotic arrangement for the list than the MTF, through its incremental conservative changes. However, its convergence rate and ability to respond to changes in the environment are poor.

A lot of research that followed Mcabe's work has attempted to combine the two algorithms, to benefit from the speed of the MTF and the accuracy of the TR. Some algorithms are hybrids of two [76], [77], [78]. Other batched methods invoke the reorganization heuristic every $k^{\text {th }}$ access [75] or after $k$ accesses in a row [79]. More recent work in this field includes the work of Schulz [80] and Bachrach et al. [81]. Randomized algorithms [82], [83], [84] are another means to reorganize lists that, generally speaking, try to move the accessed record in a more conservative way than the MTF.

Lam [85] studied the expected search cost of MTF in a dependent environment, by considering a Markovian model for dependent accesses, where there is locality of reference. Chassaing [86] showed that the expected search time for the MTF in an environment with temporal locality is not greater than any other sequential strategy. Bachrach [81] backed this result in 2002 with a comprehensive suite of experiments that tested almost all the algorithms reported in the literature. They showed that the MTF 
outperforms all other algorithms that they tested in such environments, as the dependence factor increases. This result seems to be the general consensus within the literature. To date, no other algorithm has been reported to outperform the MTF. Thus a very good metric for benchmarking the performance of an algorithm in environments with a locality of reference is to have a baseline comparison against the MTF.

\subsection{List-on-List Organization}

A common approach for algorithms dealing with environments where there is locality of reference is to minimize the access cost for the required resource as well as its surrounding resources. In a self-organizing list, this approach would reorganize some of the elements around the accessed record itself. This approach is effective in lists since the reorganization cost for a sublist is the same as that of a single record and can be achieved by updating few pointers, knowing the sublist boundaries. Such an approach has been recently published, and is referred to as a List-on-List (LOL) organization by Amer et al. [74].

A LOL algorithm is denoted by the form $\mathrm{X}-\mathrm{Y}$, where $\mathrm{X}$ would be the reorganization of the accessed record within the sublist, and $\mathrm{Y}$ would be the reorganization of the sublists among themselves. For example, consider the LOL scheme MTF-MTF. In one variation of this scheme the list is divided into number of sublists, $k$, of length $m$ each. When a requested record is found, it is moved to the front of the sublist it belongs to, and then the entire sublist is moved to the front of the list. If the sublist contains the record within the current Environment's context, we may just be lucky enough to have minimized the 
access cost for all forthcoming requests until the Environment's context changes. At the same time, by doing this, we are bringing records that are more frequently accessed within this context to the head of the list, thereby reducing the cost even more.

In order to ensure that the Environment's various contexts are preserved, a dependence capturing or partitioning automaton, alongside with the LOL reorganization algorithm can be used. This can be done by running the current queried key (at time $t$ ) with the last queried key (at time t-1) against the partitioning algorithm. If the two elements belong to the same partition within the partitioning automaton's internal representation, this automaton is rewarded and the LOL reorganization takes place. Otherwise, the automaton is penalized, and instead of performing LOL reorganization, the automaton learns a better partitioning and the sublists are modified accordingly, to match that partitioning. Recent results report two algorithms that can be used to attain such a partitioning namely, the Object Migration Automaton (OMA) [87] and the Modified Linear Reward-Penalty reinforcement rule $\left(\mathrm{ML}_{\mathrm{RP}}\right)$ [88]. A LOL scheme that uses a partitioning automaton is denoted by the form $\mathrm{X}-\mathrm{Y}-\mathrm{Z}$, where $\mathrm{Z}$ is the partitioning algorithm. One such strategy would thus be called the MTF-MTF-OMA.

To capture the dependency between the accessed queries, Amer et al. [74] used a partitioning scheme such that records of the same local context reside in the same sublist divisions. Thus, for example, they used the OMA which partitioned objects into equalsize groups. They also used the $\mathrm{ML}_{\mathrm{RP}}$ which produced different size partitions although at expense of greater time and space cost. Both schemes were based on the field of learning automata as explained below. 
OMA: The OMA partitions objects into equal-size sublists. Two different variants of the OMA algorithm are possible, each based on the time period T. If we have advance knowledge of the period $T$, we can simply move the first sublist to the end of the list after T queries. If the OMA has correctly converged, no records from this sublist are expected to be accessed except after (k-1) T queries. This method is called the MTF-MTF-OMA-P, where the $\mathrm{P}$ implies periodic. If we, however, do not know the period in advance, which is the case for web caching, the first sublist is moved after two successive queries which are deemed to not be dependent. That is, if the OMA penalizes two successive queries, we interpret that as the end of the period and move the sublist to the rear accordingly. This method is called the MTF_MTF_OMA_UP i.e., with an unknown period.

$M_{R P}$ : Unlike OMA, the $M L_{R P}$ partitions objects into different-sized groups, thereby offering more flexibility than the OMA, with comparable accuracy. However the time and space requirements are higher than that of the OMA. The MTF-MTF-ML algorithm is very similar to the MTF-MTF-OMA, except that during initialization, every record is placed in a sublist of its own. The sublists are gradually merged according to the access patterns to form larger sublists. In the $M L_{R P}$, when a record is moved from one sublist to the other, no other element is moved back to the original sublist.

The LOL approach has several benefits. Unlike the MTF, records are promoted en masse and therefore the prospects of the access time being reduced in forthcoming accesses increase. Secondly, if a record outside of the local context is accessed, it is displaced much further from the front in subsequent calls than in the MTF case, because the group of all records that obey the context may be promoted to precede it. In the case of the MTF, 
however, such an access may cause a record to linger at the front of the list for a longer duration until the entire context is moved to the front of the list, one element at a time.

\subsection{LOL in Web Caching}

As discussed in Chapter 2, since the documents requested in a given cache have locality of reference as a strong characteristic, a LOL technique can be used to achieve a nearoptimal search cost. For any given cache, since it is hard to predict the time instances at which access patterns change, we cannot use the MTF-MTF-OMA-P (periodic) approach. Instead, we could use the unknown period method. In the case of OMA, equal-size sublists are formed, which adds additional cost for moving documents to the original sublist. Since we are dealing with different size documents, if we restrict the sublist size, it is possible that the cache space is under-utilized. Instead, it is advisable to use the $\mathrm{ML}_{\mathrm{RP}}$ as the partitioning algorithm.

In the $M L_{R P}$, the partition of the cache is done into different-sized groups, which yields more flexibility than the OMA. In this algorithm, each document accessed would initially form a sublist of its own. Then, subsequently, according to the access patterns and the access history, the documents tend to organize themselves using the partition algorithm. In this algorithm, the sublists try to merge with the documents on which they are dependent, and this results in different-sized groups. Since access patterns change very frequently in the case of the Web, it is understandably crucial to have a partition algorithm which can reflect these changes. 
Since $70 \%$ of the documents accessed are one-timers, it is really important to use replacement policies wherein these documents are purged from the cache after a certain time period to avoid cache pollution. When using these partitioning algorithms along with their replacement policies, they need to work hand-in-hand so as to take care of the documents that are rarely referenced, as this effects the document hit ratio and the byte hit ratio. In order to try to attain to the optimal search cost and the document hit ratio, adaptive methods of partitioning are required, where, for every access, cache contents are self-organized.

In order to learn the access patterns for any given trace within a cache, the history of the documents should be maintained. By using the history we can group the documents with same locality of reference. When one of the documents from a particular group is referenced, we recommend that all the documents from that group be moved to the front of the list. This would result in an improved search cost for all the consecutive documents referenced from the same group. Furthermore, if any one or more of the documents of that particular group is not present in the cache, then prefetching that single or multiple documents can increase the hit ratio. If the document was wrongly partitioned out of the current working set, the partition algorithm may result in a higher search cost. Consequently, it is very crucial to decide on the right set of documents with a locality of reference, so that partitioning algorithms would perform better than those without partitioning (LRU). 


\subsection{Conclusions}

As part of the future research, the LOL strategy discussed in this Chapter could be further explored, using experiments on real trace data, so as to analyze the cache performance in terms of the search cost. The optimization of the search cost would have an impact on the performance of the document hit ratio and the byte hit ratio. We propose that an LOLbased automaton could be further studied to obtain a balance between the search cost and the document hit ratio or the byte hit ratio. We believe that the LOL technique, in conjunction with an adaptive admission and replacement policy, could effectively utilize the cache, and thus result in a better end-user experience. 


\section{Conclusions and Future Work}

\subsection{Contributions of the Thesis}

As multimedia content delivery networks, on demand applications, and services continue to grow in the wired and wireless Internet world, the latency to deliver such mission critical and cost-cum-quality sensitive services to businesses and consumers becomes even more significant. Great efforts have been made to understand changing Web traffic patterns by studying the access server logs from a variety of sources including educational, research and commercial establishments.

By surveying previous works on Web workload characteristics and cache replacement policies, we have observed that there are still many open problems in Web caching, such as a sound understanding of the workload characteristics, performance enhancing proxy placement, dynamic data caching, fault tolerant schemes, security etc. The work described in this Thesis was motivated by the need to characterize current Web workloads and to specifically see if there has been a dramatic change in the invariants behavior patterns identified in the past studies. The objective of this Thesis was three fold:

1. To understand the workload characteristics of recent server logs by separating the one-timer and non-one timer documents. 
2. To study the transfer size characteristics of one-timer and non-one timer documents in terms of their size, heavy tailed distribution and popularity, and to compare them to the total documents in each of the NLANR traces.

3. To be able to design an adaptive cache replacement policy using the respective document sizes as an admission constraint in analyzing the cache performance.

Earlier studies have shown that most web document transfers are small with the mean document size around 7 to 15 Kbytes, and the median transfer size being 2 to 3 Kbytes. However, no clear distinction has been made between one-timer and non-one timer documents by studying their file transfer size distributions. In Chapter 3 , we presented a detailed study of the Web workload characteristics. Trace driven analysis were used to study the properties of one-timer and non-one timer documents independently in detail with the help of formal mathematical models.

Weibull and lognormal distributions were found to be suitable for modeling the transfer size distributions of Web workload characteristics. The statistical properties of both fitted distributions were evaluated by examining their mean and variance for the seven traces. Based on the K-S Goodness of Fit tests, the lognormal distribution was found to be the superior fit to the empirical model obtained using the one-timer, non-one timer and total documents. By separating the one-timer and non-one timer documents, and by studying their size characteristics, we determined that both one-timer and non-one timer documents exhibit the so-called heavy tailed property. 
The popularity of non-one timer documents for each of these traces was studied, and it was found to follow a Zipf-like distribution. By studying the properties of one timer and non-one timer documents and comparing them to the total documents in each of the seven traces, we found that the Web document characteristics are predominantly influenced by their one-timer distributions, and that they exhibit self-similarity in their workloads.

In Chapter 4, we proposed a new admission control policy (LRU-AT) to study the problem of cache performance. This policy takes into account size of the requested documents and optimizes the document replacement policy to achieve a balance between the document hit ratio and the byte hit ratio.

Using trace-driven simulations, we observed that for the seven traces considered from five different proxies within NLANR, the document hit ratio was improved using the LRU-AT policy. For the lowest cache size of 4 Mbytes considered in our analysis, an improvement of $\sim 5 \%$ to $10 \%$ in hit ratio was achieved with the LRU-AT policy over the LRU and the LRU-T policies. For lower cache sizes below 4 Mbytes, we notice that LRU-AT policy achieves higher than a $10 \%$ improvement in the hit ratios compared to the LRU and the LRU-T policies. We attribute the variation in the hit ratio improvement with the LRU-AT policy, due to the NLANR proxy workload characteristics discussed in Chapter 3.

When looking at the byte hit ratio performance metric at lower cache sizes, we discovered a reverse impact using the LRU-AT policy, and this was probably due to the size-based admission constraints discussed earlier in Section 4.4.3.1. We also found that compared to the static size threshold (LRU-T) policy, the LRU-AT policy achieves a 
higher byte hit ratio. At higher cache sizes, the LRU-AT policy is able to achieve a byte hit ratio comparable to the LRU.

By tuning the size filter, LRU-AT policy dynamically adjusts to the changing access patterns to achieve a hopefully optimal hit ratio for a given cache size.

\subsection{Suggestions for Future Research}

We propose the following avenues for future research:

- In Chapter 5, we had proposed a top-level design of a learning automaton based partitioning algorithm that could be used for capturing locality of reference. We also suggested that such a self-organizing scheme could be used in conjunction with an adaptive admission and replacement policy to enhance the cache performance. Unfortunately, due to time constraints this work could not be fully undertaken. This study could be undertaken as a future project using recent experimental trace data.

- It would be advantageous to examine other Web server access logs from commercial and research organizations and lower level caches so as to understand the changes in Web server workloads.

- Due to the dynamic traffic patterns in the WWW, we suggest that a study be undertaken to understand the distribution of Web objects [89] in terms of their size and type, and the use of appropriate filters to adaptively tune the cache performance. 
In conclusion, we believe that the research frontier in enhancing Web performance lies in developing efficient, scalable, adaptive and robust Web caching schemes that can be easily adapted to existing and evolving networks. By studying the dynamics of traffic patterns and using adaptive admission constraints, it should be possible to design cache replacement policies that provide an improved end-user experience. 


\section{References}

1. A. Kodaypak and S. Dandamudi, "An Adaptive Feedback Admission Control Policy for Web Caching", Proceedings of WMSCI, the $9^{\text {th }}$ World Multi-Conference on Systemics, Cybernetics and Informatics, pp.198-203, Orlando, Florida, July 2005.

2. A. Kodaypak and S. Dandamudi, "One-Timer and Non-One-Timer Characterization of Web Traffic", Proceedings of IASSE, the $14^{\text {th }}$ International Conference on Intelligent and Adaptive Systems and Software Engineering, pp.106-111, Toronto, Canada, July 2005.

3. A. Bestavros, R. Carter, C. Cunha, A. Heddaya and S. Mirdad, "Application-Level Document Caching in the Internet", Proceedings of SDNE, the $2^{\text {nd }}$ International Workshop on Services in Distributed and Networked Environments, pp.166-173, Whistler, British Columbia, June 1995.

4. A. Balamash and M. Krunz, "An Overview of Web Caching Replacement Algorithms”, Proceedings of IEEE Communications Surveys \& Tutorials, Vol. 6, No. 2, pp. 44-56, Second Quarter 2004.

5. L. Breslau, P. Cao, L. Fan, G. Philips and S. Shenker, "Web Caching and Zipf-like Distributions: Evidence and Implication”, Proceedings of IEEE INFOCOM, pp.126134, New York City, New York, March 1999.

6. P. Barford, A. Bestavros, A. Bradley and M. E. Crovella, "Changes in Web client access patterns: Characteristics and caching implications", World Wide Web (Special 
issue on Characterization and Performance Evaluation), Vol. 2, No. 1-2, pp. 15-28, March 1999.

7. G. Abdulla, E. Fox, M. Abrams and S. Williams, "WWW Proxy Traffic Characterization with Application to Caching", Technical Report TR-97-03, Computer Science Department, Virginia Tech, March 1997.

8. S. Jin and A. Bestavros, "Temporal Locality in Web Reference Streams: Sources, Characteristics and Caching Implications (Extended Abstract)", Proceedings of ACM SIGMETRICS, pp. 110-111, Santa Clara, California, June 2000.

9. M. Arlitt, "A Performance Study of Web Servers", Master's Thesis, University of Saskatchewan, 1996.

10. J. Challenger, P.B. Danzig and A. Iyengar, "A Scalable and Highly Available System for Serving Dynamic Data at Frequently Accessed Web Sites", Technical Report RC21337, IBM Research, T.J. Watson Research Center, 1998.

11. V. Holmedahl, B. Smith and T. Yang, "Cooperative Caching of Dynamic Contents on a Distributed Web Servers", Proceedings of HPDC-7, the $7^{\text {th }}$ IEEE International Symposium on High Performance Distributed Computing, pp. 243-250, Chicago, Illinois, July1998.

12. P. Rodriguez, C. Spanner and E. W. Biersack, "Analysis of Web Caching Architectures: Hierarchical and Distributed Caching", Proceedings of IEEE/ACM Transactions on Networking, Vol. 9, No. 4, pp. 404-418, August 2001. 
13. A. Chankhunthod, P.B. Danzig, C. Neerdals, M. F. Schwartzand and K.J. Worrell, “A Hierarchical Internet Object Cache", Proceedings of the USENIX Technical Conference, pp. 153-163, San Diego, California, January 1996.

14. C. Roadknight, I. Marshall and D. Vearer, "File Popularity Characterization", Proceedings of WISP, the $2^{\text {nd }}$ Workshop on Internet Server Performance, Atlanta, Georgia, May 1999.

15. NLANR Traces: ftp://ircache.nlanr.net/Traces. Downloaded as of June 2004.

16. IRCACHE, For details see http://www.ircache.net

17. ICP, The related address is: $\underline{\text { http://icp.ircache.net/rfc2186.txt }}$

18. Squid, Related address is: http://squid.nlanr.net

19. Squid, Frequently Asked Questions: http://www.squid-cache.org/Doc/FAQ/FAQ$\underline{12 . h t m l}$.

20. Squid Internet Object Cache, For details see: http://squid.nlanr.net/squid.

21. D. Wessels and K. Claffy, "Application of Internet Cache Protocol (ICP), version 2", Internet Draft: draft-wessels-icp-v2-appl-00, Work in progress, IETF, May 1997.

22. C. Chiang, M. T. Liu and M. E. Muller, "Caching Neighborhood Protocol: A Foundation for Building Dynamic Web Caching Hierarchies with Proxy Servers", Proceedings of ICPP, the International Conference on Parallel Processing, pp. 516524, Aizu, Japan, September 1999. 
23. D. Povey and J. Harrison, "A Distributed Internet Cache", Proceedings of the $20^{\text {th }}$ Australian Computer Science Conference, Sydney, Australia, February 1997.

24. V. Valloppillil and K. W. Ross, "Cache Array Routing Protocol v1.1. Internet Draft", http://ds1.internic.net/internet-drafts/draft-vinod-carp-v1-03.txt, February 1998.

25. R. Tewari, M. Dahlin, H. M. Vin and J.S. Kay, "Beyond Hierarchies: Design Considerations for Distributed Caching on the Internet", Proceedings of the ICDCS, the $19^{\text {th }}$ International Conference on Distributed Computing Systems, pp. 273-284, Austin, Texas, May 1999.

26. S. Gadde, M. Rabinovich and J. Chase, "Reduce, Reuse, Recycle: An Approach to Building Large Internet Caches", Proceedings of the Workshop on Hot Topics in Operating systems, pp. 93-98, May 1997.

27. Z. Wang, "Cachemesh: a Distributed Cache System for World Wide Web", NLANR Web Cache Workshop, Boulder, Colorado, June 1997.

28. L. Fan, P. Cao, J. Almedia and A. Broder, "Summary Cache: A Scalable Wide-Area Web Cache sharing Protocol”, SIGCOMM, pp.254-265, Feb 1998.

29. A. Rousskov and D. Wessels, "Cache Digest", Proceedings of $3^{\text {rd }}$ International WWW Caching Workshop, pp. 272-273, Manchester, UK, June 1998.

30. Relais: Cooperative Caches for the World Wide Web, 1998. (http://wwwsor.inria.fr/projects/relais/). 
31. M. Rabinovich, J. Chase and S.Gadde, "Not all Hits are Created Equal: Cooperative Proxy Caching over a Wide Area Network" Computer Networks and ISDN Systems Vol. 30, No. 22-23, pp. 2253-2259, November 1998.

32. M. Abrams, C. R. Standridge, G. Abdulla, S. Williams and E. A. Fox, "Caching Policies: limitations and potentials", Proceedings of the $4^{\text {th }}$ International WWW Conference, Boston, Massachusetts, December 1995.

33. V. N. Padmanabhan and J. C. Mogul, "Using Predictive Prefetching to Improve World-Wide Web Latency", ACM SIGCOMM Computer Communication Review, Vol. 26, No. 3, pp. 22-36, July 1996.

34. A. Bestavros and C. Cunha, "Server-Initiated Document Dissemination for the WWW”, IEEE Data Engineering Bulletin, Vo. 19, No. 3, pp. 3-11, September 1996.

35. T. Palpanas and A. Mendelzon, "Web prefetching using partial match Prediction", Proceedings of the $4^{\text {th }}$ International Web Caching Workshop, San Diego, California, March 1999.

36. C.R. Cunha and C. F. B. Jaccoud, "Determining WWW User's Next Access and its Application to Prefetching", Proceedings of $2^{\text {nd }}$ IEEE Symposium on Computers and Communications, Alexandria, Egypt, July 1997.

37. M. Crovella and P. Batford, "The Network Effects of Prefetching", Proceedings of the IEEE INFOCOM, San Francisco, California, 1998. 
38. T. M. Kroeger, D.D. E. Long and J. C. Mogul, "Exploring the Bounds of Web latency Reduction from Caching and Prefetching", Proceedings of the USENIX Symposium on Internet Technologies and Systems, pp. 13-22, Monterey, California, December 1997.

39. E. P. Markatos and C.E. Chronaki, "A Top-10 Approach to Prefetching on Web", Proceedings of Internet Society Conference, Geneva, Switzerland, July 1998.

40. E. Cohen, B. Krishnamurthy and J. Rexford, "Evaluating Server-Assisted Cache Replacement in the Web", Proceedings of the $6^{\text {th }}$ European Symposium on Algorithms, pp. 307-319, 1998.

41. K. Chinen and S. Yamaguchi, "An Interactive Prefetching Proxy Server for Improvement of WWW latency", Proceedings of the $7^{\text {th }}$ Annual Conference of the Internet Society, Kuala Lampur, Malaysia, June 1997.

42. M. Gwertzman and J. Seltzer, "An analysis of geographical push caching". http://www.eecs.harvard.edu/.

43. T. S. Loon and V. Bharghavan, "Alleviating the Latency and Bandwidth Problems in WWW Browsing", Proceedings of the USENIX Symposium on Internet Technologies and Systems, pp. 219-230, Monterey, California, December 1997.

44. L. Fan,P. Cao, W. Lin and Q. Jacobson, "Web Prefetching between Low-bandwidth Clients and Proxies: Potential and Performance", Proceedings of the ACM SIGMETRICS Conference, pp, 178-187, Atlanta, Georgia, May 1999. 
45. M. Arlitt and C. Williamson, "Web Server Workload Characterization: The Search for Invariants", Proceedings of the ACM SIGMETRICS Conference, pp. 126-137, Philadelphia, Pennsylvania, April 1996.

46. M. Arlitt and C. Williamson, "Trace-Driven Simulation of Document Caching Strategies for Internet Web servers", The Society for Computer Simulation Journal, Vol. 68, No. 1, pp. 23-33, January 1997.

47. M. Arlitt and C. Williamson, "Internet Web Servers: Workload Characterization and Performance Implications", IEEE/ACM Transactions on Networking, Vol. 5, No. 5, pp. 631-645, October 1997.

48. M. Busari and C. Williamson, "On Sensitivity of Web Proxy Cache Performance to Workload Characteristics", Proceedings of IEEE INFOCOM, pp. 1225-1234, Anchorage, Alaska, April 2001.

49. C. Cunha, A. Bestavros and M. Crovella, "Characteristics of WWW Client-based Traces", Technical Report TR-95-010, Department of Computer Science, Boston University, July 1995.

50. A. Mahanti, Web Proxy Workload Characterization and Modeling, M.Sc. Thesis, Department of Computer Science, University Of Saskatchewan, September 1999.

51. A. Mahanti, C. Williamson and D. Eager, "Traffic Analysis of a Web Proxy Caching Hierarchy", IEEE Network, pp. 16-23, May/June 2000. 
52. G. K. Zipf, "Human Behavior and the Principle of Least-Effort", Addison-Wesley, Cambridge, Massachusetts, 1949.

53. V. Almeida, A. Bestovros, M. Crovella and A. Oliveira, "Characterizing Reference Locality in the WWW", Proceedings of IEEE/ACM International Conference on Parallel and Distributed Information Systems, pp. 92-107, Miami, Florida, December 1996.

54. J. Sprin, “Distance String Models for Program Behavior”, IEEE Computer, Vol. 9, No.11, pp. 14-20, November 1976.

55. M. Arlitt, R. Friedrich and T. Jin, "Performance Evaluation of Web proxy Cache Replacement Policies", Technical Report HPL-98-97, HP Labs, May 1998.

56. S. Williams et al., "Removal Policies in Network Caches for World-Wide-Web Documents", Proceedings of ACM SIGCOMM conference on Applications, Technologies, Architectures and Protocols for Computer Communication, pp. 293305, Stanford, California, August 1996.

57. M. Crovella and A. Bestavros, "Self-Similarity in World Wide Web traffic: Evidence and possible causes", Proceedings of the Sigmetrics Conference on Measurement and Modeling of Computer Systems, pp. 160-169, Philadelphia, Pennsylvania, May 1996.

58. C. Aggarwal, J.L Wolf and P.S. Yu, "Caching on the World Wide Web", IEEE Transactions on Knowledge and Data Engineering, Vol. 11, No. 1, pp. 94-106, January/Febuary 1999. 
59. C. M. Bowman, P.B. Danzig, D. R. Hardy, U. Manber and M. F. Schwartz, "Harvest: A Scalable, Customizable Discovery and Access System", Technical Report CU-CS732-95, University of Colorado, Department Of Computer Science, Boulder, Colorado, March 1995.

60. R. Wooster and M. Abrams, "Proxy Caching that Estimates Page Load delay", Proceedings of the $6^{\text {th }}$ International WWW Conference, pp. 325-334, Santa Clara, California, April 1997.

61. J. Robinson and M. Devarkonda, "Data Cache Management Using Frequency Based Replacement", Proceedings of the ACM SIGMETRICS Conference on the Measurement and Modeling of Computer Systems, pp. 134-142, Boulder, Colorado, May 1990.

62. D. Willick, D. Eager and R. Bunt, "Disk Cache Replacement Policies for Network Fileservers", Proceedings of the $13^{\text {th }}$ International Conference on Distributed Computing Systems, pp. 2-11, Pittsburgh, Pennsylvania, May 1993.

63. N. Niclausse, Z. Liu and P. Nain, "A New Efficient Caching Policy for the World Wide Web", Proceedings of WISP, the Workshop on Internet Server Performance, pp. 119-128, Madison, Wisconsin, June 1998.

64. Pei Cao and Sandy Irani, "Cost-aware WWW proxy caching algorithms", Proceedings of the USENIX Symposium on Internet Technology and Systems, pp. 193-206, Monterey, California, December 1997. 
65. N. E. Young, "The K-server Dual and Loose Competitiveness for Paging", Algorithmica, Vol. 11, No. 6, pp. 535-541, June 1994.

66. S. Jin and A. Bestavros, "Popularity-Aware Greedy Dual-Size Web Proxy Caching Algorithms", Proceedings of ICDCS, the $20^{\text {th }}$ International Conference on Distributed Computing Systems, pp. 254-261, Taipei, Taiwan, April 2000.

67. R. Wooster, "Optimizing Response Time, Rather than Hit Rates of WWW Caches", M. S. thesis, Computer Science Department, Virginia Tech, December 1996.

68. National Lab for Applied Network Research, Sanitized Access log, Available at ftp://ircache.nlanar.net/Traces/,July 2004.

69. P. Barford and M. Crovella, "Generating Representative Web Workloads for Network and Server Performance Evaluation", Proceedings of ACM SIGMETRICS, pp.151160, Madison, Wisconsin, June 1998.

70. B. Krishnamurthy and J. Rexford, "Software Issues in Characterizing Web Server Logs", W3C Web Characterization Group Workshop, Boston, Massachusetts, November 1998.

71. C. Williamson, "On Filter Effects in Web Caching Hierarchies”, ACM Transactions on Internet Technology, Vol. 2, No. 1, pp. 47-77, February 2002.

72. A.M. Law and W.D. Kelton, "Simulation Modeling and Analysis", Third Edition, McGraw-Hill, pp.292-402, Boston, 2000. 
73. P. Lorenzetti, L. Rizzo and L. Viscisano, "Replacement policies for a proxy cache", (http://www.iet.unipi.it/luigi/research.html)

74. A. Amer and B.J. Oommen, "Lists on Lists: A Framework for Self-organizing Lists in Environments with Locality of Reference", Proceedings of WEA, the International Workshop on Experimental Algorithms, pp.109-120, Spain, May 2006.

75. J. McCabe, "On serial files with relocatable records", Operations Research, Vol. 12, pp. 609-618, 1965.

76. J. R. Bitner, "Heuristics that dynamically organize data structures", SIAM Journal on Computing, Vol. 8, pp. 82-110, 1979.

77. R. Rivest, "On self-organizing sequential search heuristics", Communications of the ACM, Vol. 19, pp. 63-67, 1976.

78. A. M. Tenenbaum and R. M. Nemes, "Two spectra of self-organizing sequential search algorithms", SIAM Journal on Computing, Vol. 11, pp. 557-566, 1982.

79. Y. C. Kan and S. M. Ross, "Optimal list order under partial memory constraints", Journal of Applied Probability, Vol. 17, pp. 1004-1015, 1980.

80. F. Schulz, "Two new families of list update algorithms", Proceedings of ISAAC, the $9^{\text {th }}$ International Symposium on Algorithms and Computation, pp. 99-108, 1998. 
81. R. Bachrach, R. El-Yaniv and M. Reinstadtler, "On the competitive theory and practice of online list accessing algorithms", Algorithmica, Vol. 32, pp. 201-245, 2002.

82. S. Irani, "Two results on the list update problem", Information Processing Letters, Vol. 38, pp. 301-306, 1991.

83. N. Reingold, J. Westbrook and D. D. Sleator, "Randomized competitive algorithms for the list update problem", Algorithmica, Vol. 11, pp. 15-32, 1994.

84. S. Albers, "Improved randomized on-line algorithms for the list update problem", SIAM Journal on Computing, Vol. 27, pp. 670-681, 1998.

85. K. Lam, M. Y. Leung and M. K. Siu, "Self-organizing files with dependent accesses", Journal of Applied Probability, Vol. 21, pp. 343-359, 1984.

86. P. Chassaing, "Optimality of move-to-front for self-organizing data structures", Annals of Applied Probability, Vol. 3, pp. 1219-1240, 1993.

87. B. J. Oommen and D. C. Y. Ma, "Deterministic learning automata solutions to the equipartitioning problem", IEEE Transactions on Computers, Vol. 37, 1988.

88. B. J. Oommen and D. C. Y. Ma, "Stochastic automata solutions to the object partitioning problem", The Computer Journal, Vol. 35, pp. A105-A120, 1992.

89. A. Abhari, "Web Object-Based Policies for Managing Proxy Caches", Ph.D Thesis, School Of Computer Science, Carleton University, July 2003. 